Document downloaded from:

http://hdl.handle.net/10251/148101

This paper must be cited as:

Alpuente Frasnedo, M.; Ballis, D.; Sapiña-Sanchis, J. (2019). Static correction of Maude programs with assertions. Journal of Systems and Software. 153:64-85.

https://doi.org/10.1016/j.jss.2019.03.061

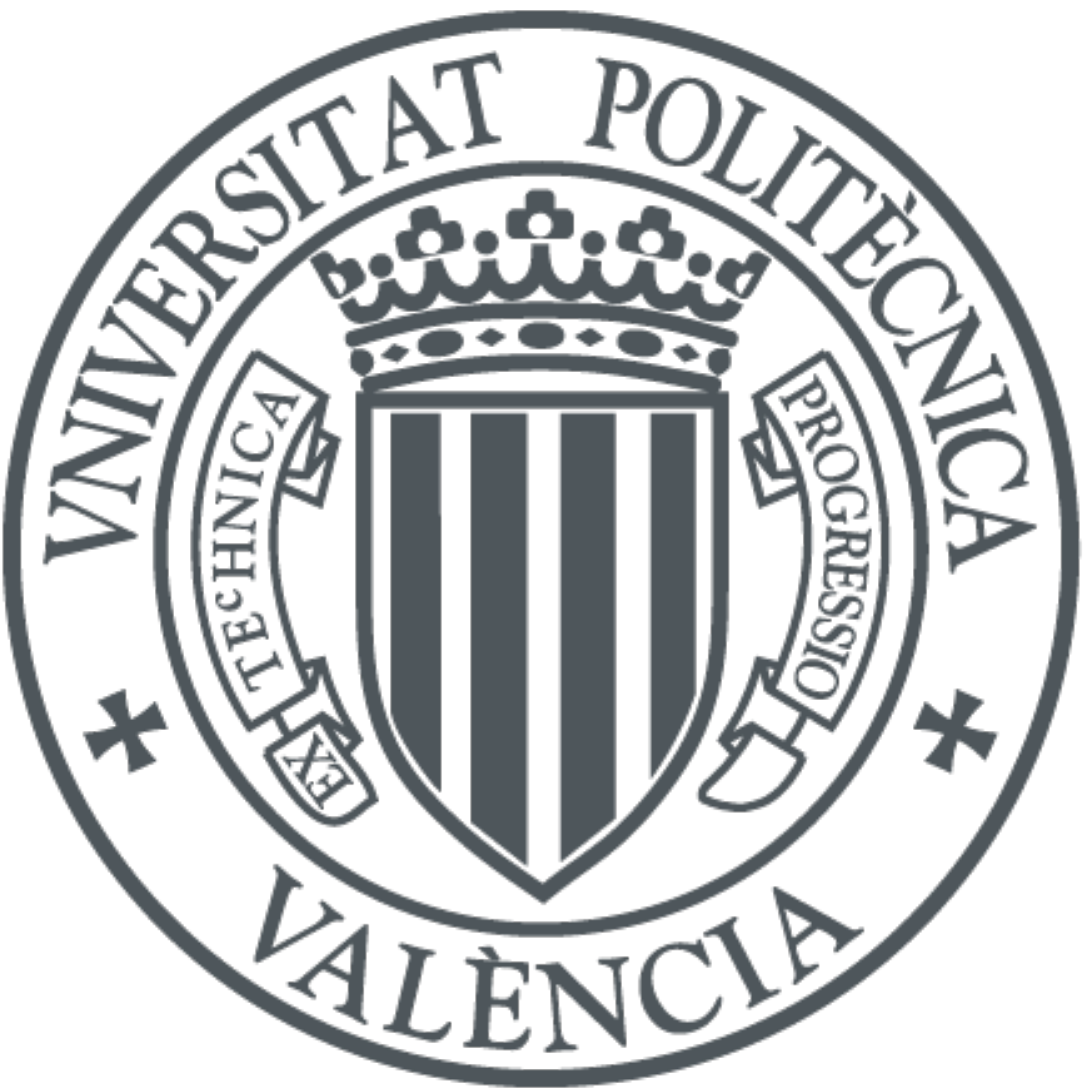

The final publication is available at

https://doi.org/10.1016/j.jss.2019.03.061

Copyright Elsevier

Additional Information 


\title{
Static Correction of Maude Programs with Assertions ${ }^{\text {th }}$
}

\author{
M. Alpuente ${ }^{\mathrm{a}}$, D. Ballis ${ }^{\mathrm{b}}$, J. Sapiña $a^{\mathrm{a}, *}$ \\ ${ }^{a}$ DSIC-ELP, Universitat Politècnica de València \\ Camino de Vera s/n, Apdo 22012, 46071 Valencia, Spain \\ ${ }^{b}$ DIMI, University of Udine, \\ Via delle Scienze, 206, 33100, Udine, Italy
}

\begin{abstract}
In this paper, we present a novel transformation method for Maude programs featuring both automatic program diagnosis and correction. The input of our method is a reference specification $\mathcal{A}$ of the program behavior that is given in the form of assertions together with an overly general program $\mathcal{R}$ whose execution might violate the assertions. Our correction technique translates $\mathcal{R}$ into a refined program $\mathcal{R}^{\prime}$ in which every computation is also a computation in $\mathcal{R}$ that satisfies the assertions of $\mathcal{A}$. The technique is first formalized for topmost rewrite theories, and then we generalize it to larger classes of rewrite theories that support nested structured configurations. Our technique copes with infinite space states and does not require the knowledge of any failing run. We report experiments that assess the effectiveness of assertion-driven correction.
\end{abstract}

Keywords: program repair, assertion checking, program transformation, rewriting logic, equational rewriting, Maude

\section{Introduction}

Assertion checking is a practical means of validating programs. Program assertions often specify "can never happen" conditions that help detect pro-

\footnotetext{
This work has been partially supported by the EU (FEDER) and the Spanish MINECO under grant TIN2015-69175-C4-1-R, and by Generalitat Valenciana PROMETEOII/2015/013 and PROMETEO/2019/098.

${ }^{*}$ Corresponding author

Email addresses: alpuente@dsic.upv.es (M. Alpuente), demis.ballis@uniud.it (D. Ballis), jsapina@dsic.upv.es (J. Sapiña)
} 
gram faults by testing, via runtime-checking, that the implementation behaves as intended on specific runs. Techniques to automatically detect discrepancies with the expected behavior given by the assertions are slowly making their way into industrial practice, yet the automated generation of valid program fixes is still a challenging problem with practical techniques having arisen only recently [20].

Maude [6] is a high-level programming language and system that efficiently implements Rewriting Logic [17], which is a logic of change that seamlessly unifies a wide variety of models of concurrency. Thanks to its logical basis, Maude provides a precise mathematical model that allows it to be used as a programming language and as a formal verification system. The language integrates: 1) functional, concurrent, logic, and object-oriented computations; 2) rich type structures with sorts, subsorts, and operator overloading; 3) equational reasoning modulo axioms such as commutativity, associativitycommutativity, and associativity-commutativity-identity of functions, which efficiently supports automated reasoning with typed data structures such as lists, sets, and multisets, and typical hierarchical/structural relations such as $i s_{-} a$ and part_of. Maude is implemented as a high-performance interpreter (up to 2.98 million rewrites per second on a standard computer); a compiler is under development that brings this number up to dozens of millions of rewrites per second [7]. Because of its efficient rewriting engine and its metalanguage capabilities, Maude turns out to be an excellent instrument to create rich executable environments for various logics, programming languages, and tools.

Maude's formal tools are numerous and perform different analysis and verification tasks, either statically (e.g., Maude's theorem prover and model checker [6]) or dynamically (Maude's assertion checker [3, 2]). However, to the best of our knowledge, there is no theoretical foundation for integrating assertions into a general methodology for automated program correction in Maude. While the research on automated diagnosis of Maude programs has recently made substantial progress (see [3]), effective fault localization, debugging, and repair still pose some important challenges. In fact, although checking assertions at runtime greatly facilitates finding program bugs, it increases the overall execution time and the induced repairs heavily depend on specific failing runs. Static, automated program repair does not depend on concrete execution traces and has the potential to achieve significant cut downs on the cost of improving software quality, since no special, and timeconsuming runtime infrastructure is needed to deliver program fixes. 
Our correction transformation works with Maude programs, that is, rewrite theories of the form $\mathcal{R}=(\Sigma, E, R)$, where $E$ is a (confluent and terminating) set of equations and $R$ is a set of rules that is coherent w.r.t. the equations [8] (intuitively, this ensures that a rewrite step with $R$ can always be postponed in favor of deterministically rewriting with $E$ ). In our methodology, rewrite theories are equipped with system assertions, with each assertion consisting of a pair $\Pi \mid \varphi$, where $\Pi$ (the state template) is a term and $\varphi$ (the state invari$a n t$ ) is a quantifier-free first-order formula with equality. Intuitively, system assertions specify those states such that, for every subterm of the state which fits the algebraic structure of $\Pi$ with pattern matching substitution $\sigma$, the constraints given by $\varphi \sigma$ are satisfied.

Roughly speaking, a computation in a rewrite theory $\mathcal{R}$ is a sequence of states $\mathcal{C}$, where each state transition in the sequence $\mathcal{C}$ is computed by an application of a rewrite rule of $\mathcal{R}$. Given a set of system assertions $\mathcal{A}$, the notion of correction we seek is based on a binary relation $\leq \mathcal{A}$ on rewrite theories, such that $\mathcal{R}^{\prime} \leq \mathcal{A} \mathcal{R}$ when $\mathcal{R}^{\prime}$ is a correction of $\mathcal{R}$ w.r.t. $\mathcal{A}$, meaning that $\mathcal{R}^{\prime}$ is a valid strenghtening of $\mathcal{R}$ that enforces $\mathcal{A}$. More precisely, $\mathcal{R}^{\prime} \leq \mathcal{A}$ $\mathcal{R}$ holds when: 1) every computation of $\mathcal{R}^{\prime}$ is a computation of $\mathcal{R}$ (i.e., no spurious states are produced); and 2) every assertion in $\mathcal{A}$ is satisfied by all states that appear in the computations of $\mathcal{R}^{\prime}$. Note that points 1 and 2 ensure that $\mathcal{R}^{\prime}$ is maximal in the sense that only those computations of $\mathcal{R}$ that contain assertion violations are removed from $\mathcal{R}^{\prime}$.

Our correction transformation essentially works as follows. Starting from an overly general rewrite theory $\mathcal{R}=(\Sigma, E, R)$ (that is, a rewrite theory that contains all desired computations but may disprove some of the assertions), $\mathcal{R}$ is coerced by inserting suitable conditions (abetted by the assertions of $\mathcal{A}$ ) in the rules of $R$ until a suitable correction is reached that satisfies all the assertions. The inserted conditions are defined by means of new equations that are added to $E$.

An important feature of our technique is that it applies to Full Maude [6], which is a powerful extension of Maude that is written in Maude itself and that gives support for object-oriented specification and advanced module operations. Particular care is put in the transformation to ensure both that the new equations added to $E$ do not break Maude's executability requirements, i.e., Church-Rosser property, (operational) termination, and coherence, and that the transformed rules do not introduce spurious computations. The correction itself does not introduce new discrepancies or regressions, that is, repairing an assertion cannot cause the failure of another assertion down- 
stream.

The automated correction technique that we propose in this paper is beyond the capabilities of current Maude tools. Furthermore, our technique provides semantic guarantees on the corrections: they do not remove good runs and reduce the number of failing assertions to zero. Since all assertions are always satisfied after the correction transformation for all inputs (rather than just for specific executions), runtime assertion checking can be completely omitted after the correction. This eliminates the need for massive assertion testing required by [3, 2] and improves both the safety and efficiency of programs.

A different, preliminary approach for automated program repair was sketched in 2] that is dynamic (it relies on concrete failing computations) and does not apply to undiscovered bugs, in contrast to the static correction transformation proposed in this work. Also, as a major advantage of this work, the verified program corrections are computed without resorting to time-expensive, monitored runtime environments.

Plan of the paper. After some technical preliminaries in Section 2, we introduce a running example that is used along the paper to illustrate the kind of corrections we aim to produce automatically. Section 3 summarizes our system assertion language, which allows safety properties $\Pi \mid \varphi$ to be defined expressing that any subterm of a reachable state that fits the pattern given by $\Pi$ has to invariably satisfy $\varphi$. Section 4 encodes a checking mechanism for system assertions as an expanded equational theory $\mathcal{E}^{\mathcal{A}}$ (extending the original theory $\mathcal{E}$ ) that catches every possible assertion violation w.r.t. the assertion set $\mathcal{A}$. Section 5 formalizes our verified correction technique for topmost rewrite theories. Essentially, from the constraints given by the assertions, we synthesize correcting rule conditions that invoke the new functions defined in $\mathcal{E}^{\mathcal{A}}$. These conditions are used to proactively enable/disable the rule computations that are responsible for the undesired behaviors without the need for monitoring the program execution at runtime. Section 6 extends the correction methodology to more complex rewrite theories, including topmost theories modulo structural axioms such as associativity, commutativity and unity, and to Russian doll theories, which are structured in a more sophisticated, inductively nested way. Section 7 presents an experimental evaluation of a practical tool, called ÁTAME, that implements our program correction methodology. Its effectiveness is measured from several points of view: code size, execution time, as well as program transformation time. Section 8 dis- 
cusses some related work and it concludes. Proofs of all technical results are given in the Appendix.

\section{Equational Theories and Rewrite Theories}

Let us recall some important notions that are relevant to this work. We assume some basic knowledge of term rewriting [24] and Rewriting Logic [17]. Some familiarity with the Maude language [5, 6] is also required.

Maude is a Rewriting Logic [17] specification and verification system whose operational engine is mainly based on a very efficient implementation of rewriting. Maude's basic programming statements are equations and rewrite rules. Equations express deterministic computations leading to a unique final result, and are used to model system states as terms of an algebraic data type. Rules express transitions between states and are used to naturally express concurrent, nondeterministic, and possibly nonterminating system computations.

We consider an (order-sorted) signature $\Sigma$ of operators (i.e, function symbols), with a finite poset of sorts $(S,<)$ that models the usual subsort relation [5]. The connected components of $(S,<)$ are the equivalence classes $[s]$ corresponding to the least equivalence relation $\equiv_{<}$containing $<$. We assume that each equivalence class of sorts contains a top sort that is a supersort of every other sort in the class. Given a sort $s, \operatorname{top}(s)$ denotes the top sort of $s$. Additionally, we assume that the signature $\Sigma$ includes a distinguished sort $\top$ (similar to Maude's Universal sort) that types every possible term ${ }^{1}$. The $\top$ sort is used to define auxiliary, polymorphic, ad-hoc overloaded operators for each connected component in the theory that can be applied to every term independently of its specific sort.

An operator $f$ of $\Sigma$ in prefix notation is specified by notation $f: s_{1} \ldots s_{n} \rightarrow$ $s, n \geq 0$, where $s_{1} \ldots s_{n}$ denotes the sequence of argument sorts (that is, the arity of $f$ ), and $s$ is the sort of the return value. When the arity of $f$ is the empty sequence, $f$ is called constant. An operator of $\Sigma$ in mixfix notation can be specified by using underscores as place holders for the input arguments (e.g. $\left.\otimes_{-}: s_{1} s_{2} \rightarrow s\right)$. A finite, possibly empty, sequence of sorts is denoted by $\vec{s}$.

\footnotetext{
${ }^{1}$ Actually, in Maude, $\top$ is specified by the keyword Universal, which does not denote a real sort; it is instead a place holder for any known sort.
} 
We also consider an $S$-sorted family $\mathcal{V}=\left\{\mathcal{V}_{s}\right\}_{s \in S}$ of disjoint variable sets. $\mathcal{T}(\Sigma, \mathcal{V})_{s}$ and $\mathcal{T}(\Sigma)_{s}$ are the sets of terms and ground terms of sort $s$, respectively. We write $\mathcal{T}(\Sigma, \mathcal{V})$ and $\mathcal{T}(\Sigma)$ for the corresponding term algebras. The set of variables that occur in a term $t$ is denoted by $\mathcal{V}$ ar $(t)$. By notation $x: s$, we denote that variable $x$ has sort $s$. A simple syntactic condition on $\Sigma$ and $(S,<$ ), called preregularity [5], ensures that each (wellformed) term $t$ always has a least sort possible among all sorts in $S$, which is denoted by $l s(t)$. More precisely, a signature $\Sigma$ is preregular if for each function symbol definition $f: s_{1}, \ldots, s_{n} \rightarrow s \in \Sigma$, with $n \geq 1$, and any sequence $s_{1}^{\prime} \ldots s_{n}^{\prime}$ with $s_{i}^{\prime} \leq s_{i}$ for all $i$, the term $f\left(x_{1}: s_{1}^{\prime}, \ldots, x_{n}: s_{n}^{\prime}\right)$ has a unique least sort. We assume that order-sorted signatures are preregular.

A position $w$ in a term $t$ is represented by a sequence of natural numbers that addresses a subterm of $t$ ( $\Lambda$ denotes the empty sequence, i.e., the root position). Given a term $t$, we let $\mathcal{P} o s(t)$ denote the set of positions of $t$. By $t_{\mid w}$, we denote the subterm of $t$ at position $w$, and by $t[u]_{w}$, we denote the result of replacing the subterm $t_{\mid w}$ by the term $u$ in $t$. By root $(t)$, we denote the operator of $t$ that occurs at position $\Lambda$.

A substitution $\sigma \equiv\left\{x_{1} / t_{1}, x_{2} / t_{2}, \ldots, x_{n} / t_{n}\right\}$ is a mapping from the set of variables $\mathcal{V}$ to the set of terms $\mathcal{T}(\Sigma, \mathcal{V})$, which is equal to the identity everywhere except for a set of variables $\left\{x_{1}, \ldots, x_{n}\right\}$. By $\varepsilon$, we denote the identity substitution. The application of a substitution $\sigma$ to a term $t$, denoted $t \sigma$, is defined by induction on the structure of terms:

$$
t \sigma= \begin{cases}x \sigma & \text { if } t=x, x \in \mathcal{V} \\ f\left(t_{1} \sigma, \ldots, t_{n} \sigma\right) & \text { if } t=f\left(t_{1}, \ldots, t_{n}\right), n \geq 0\end{cases}
$$

Given two terms $s$ and $t$, a substitution $\sigma$ is the matcher of $t$ in $s$, if $s \sigma=t$. The term $t$ is an instance of the term $s$, iff there exists a matcher $\sigma$ of $t$ in $s$. Given two substitutions $\theta$ and $\theta^{\prime}$, their composition $\theta \theta^{\prime}$ is defined as $t\left(\theta \theta^{\prime}\right)=(t \theta) \theta^{\prime}$ for every term $t$. We recall that composition is associative. Given a binary relation $\rightsquigarrow$, we denote the usual transitive (resp., transitive and reflexive) closure of $\rightsquigarrow$ by $\rightsquigarrow^{+}$(resp., $\left.\rightsquigarrow^{*}\right)$.

A labelled conditional equation, or simply (conditional) equation, is an expression of the form $[l]: \lambda=\rho$ if $C$, where $l$ is a label (i.e., a name that identifies the equation), $\lambda, \rho \in \mathcal{T}(\Sigma, \mathcal{V})$ (with $l s(\lambda) \equiv<l s(\rho)$ ), and $C$ is a (possibly empty) sequence $c_{1} \wedge \ldots \wedge c_{n}$, where each $c_{i}$ is a Boolean 
expression ${ }^{2}$, When the condition $C$ is empty, we simply write $[l]: \lambda=\rho$.

A labelled conditional rewrite rule, or simply (conditional) rule, is an expression of the form $[l]: \lambda \Rightarrow \rho$ if $C$, where $l$ is a label, $\lambda, \rho \in \mathcal{T}(\Sigma, \mathcal{V})$ (with $l s(\lambda) \equiv_{<} l s(\rho)$ ), and $C$ is a (possibly empty) conjunction of Boolean expressions $\left.c_{1} \wedge \ldots \wedge c_{n}\right]^{3}$ When the condition $C$ is empty, we simply write $[l]: \lambda \Rightarrow \rho$.

When no confusion can arise, rule and equation labels $[l]$ are often omitted. The term $\lambda$ (resp., $\rho$ ) is called left-hand side (resp. right-hand side) of the rule $\lambda \Rightarrow \rho$ if $C$ (resp. equation $\lambda=\rho$ if $C$ ).

Roughly speaking, a conditional rewrite theory [17] seamlessly combines a set $R$ of conditional rewrite rules (or conditional term rewriting system, CTRS), with an equational theory $\mathcal{E}$ (also possibly conditional) that may include ordinary equations and axioms, i.e., distinguished equations expressing algebraic laws such as associativity (A), commutativity (C), and unity (U) of function symbols. Within this framework, the system states are typically represented as elements of an algebraic data type that is specified by the equational theory, while the system computations are modeled via the rewrite rules, which describe transitions between states and are applied modulo the equations and axioms.

More formally, an (order-sorted) equational theory $\mathcal{E}$ is a pair $(\Sigma, E)$, where $\Sigma$ is an order-sorted signature, $E=\Delta \cup B$ with $\Delta$ being a collection of (oriented) conditional equations and $B$ a collection of equational axioms such as associativity, commutativity, and unity that can be associated with any binary operator of $\Sigma$. The equational theory $\mathcal{E}$ induces a congruence relation on the term algebra $\mathcal{T}(\Sigma, \mathcal{V})$, which is denoted by $={ }_{E}$.

A conditional rewrite theory (or simply, rewrite theory) is a triple $\mathcal{R}=$ $(\Sigma, E, R)$, where $(\Sigma, E)$ is an order-sorted equational theory and $R$ is a set of conditional rewrite rules.

\subsection{Modeling Concurrent Systems in Maude: Our running example}

Concurrent systems can be formalized as rewrite theories. In Maude, rewrite theories are encoded by means of system modules, which are syn-

\footnotetext{
${ }^{2}$ Actually, Maude supports different kinds of conditions in equations such as equational conditions, membership tests, and matching conditions. Nonetheless, all of them can be interpreted as Boolean expressions whose canonical form is a truth value.

${ }^{3}$ Note that we prevent rule conditions from including Maude rewrite expressions, since they are not currently supported by our correction technique.
} 
tactic containers that list the signature (i.e. sorts, subsorts, and operators along with their axioms), and the variables in play, as well as the equations and rewrite rules of the rewrite theory to be specified. As for the algebraic axioms, they are indirectly expressed in Maude as attributes of their corresponding operator (using the assoc, comm and id: keywords) and are used $^{4}$ for $B$-matching. The user is unburdened from having to give explicit equational definitions of some operators (e.g., equality $==$, inequality $=/=$, arithmetic operations) since Maude provides them in a built-in way. Each syntactic element in the module is declared by using a rather intuitive keyword. For instance, sorts and subsorts are specified by keywords sort and subsort, operators by op, conditional equations and rules by ceq and crl, while unconditional equations and rules are specified by eq and $r l$. Keywords sorts and ops are abbreviations that can be used to declare multiple sorts and operators in a single line. Finally, the ctor attribute is used to specify constructor operators that are used to define program data structures.

The following Maude program will be used as a running example throughout the paper.

\section{Example 2.1}

The following Maude system module CONTAINER-TERMINAL encodes a rewrite theory that formalizes a concurrent system that models cargo manipulation in a container terminal. We simplify the model by disregarding some details that are irrelevant to our discussion such as the existence of unique container identifiers or the alignment of ship and cargo destinations.

Maude has very liberal views on identifiers which provides a very flexible syntax when combined with mixfix notation. However, it has consequences; the most obvious is its sensitivity to whitespace, which requires writing blank characters around all of the key tokens and even before the terminal '.' that ends each statement.

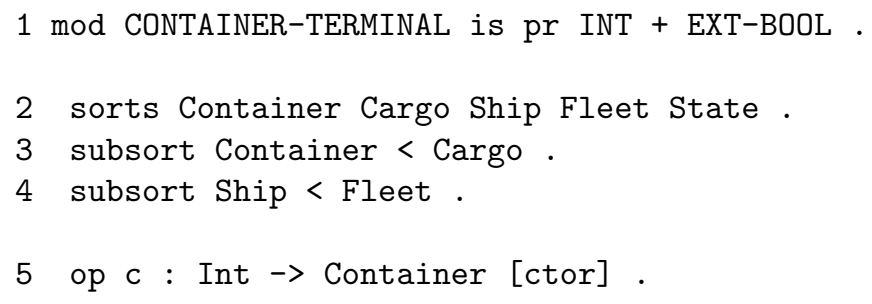

${ }^{4}$ In Maude, algebraic axioms are also used for parsing and for theory normalization. 


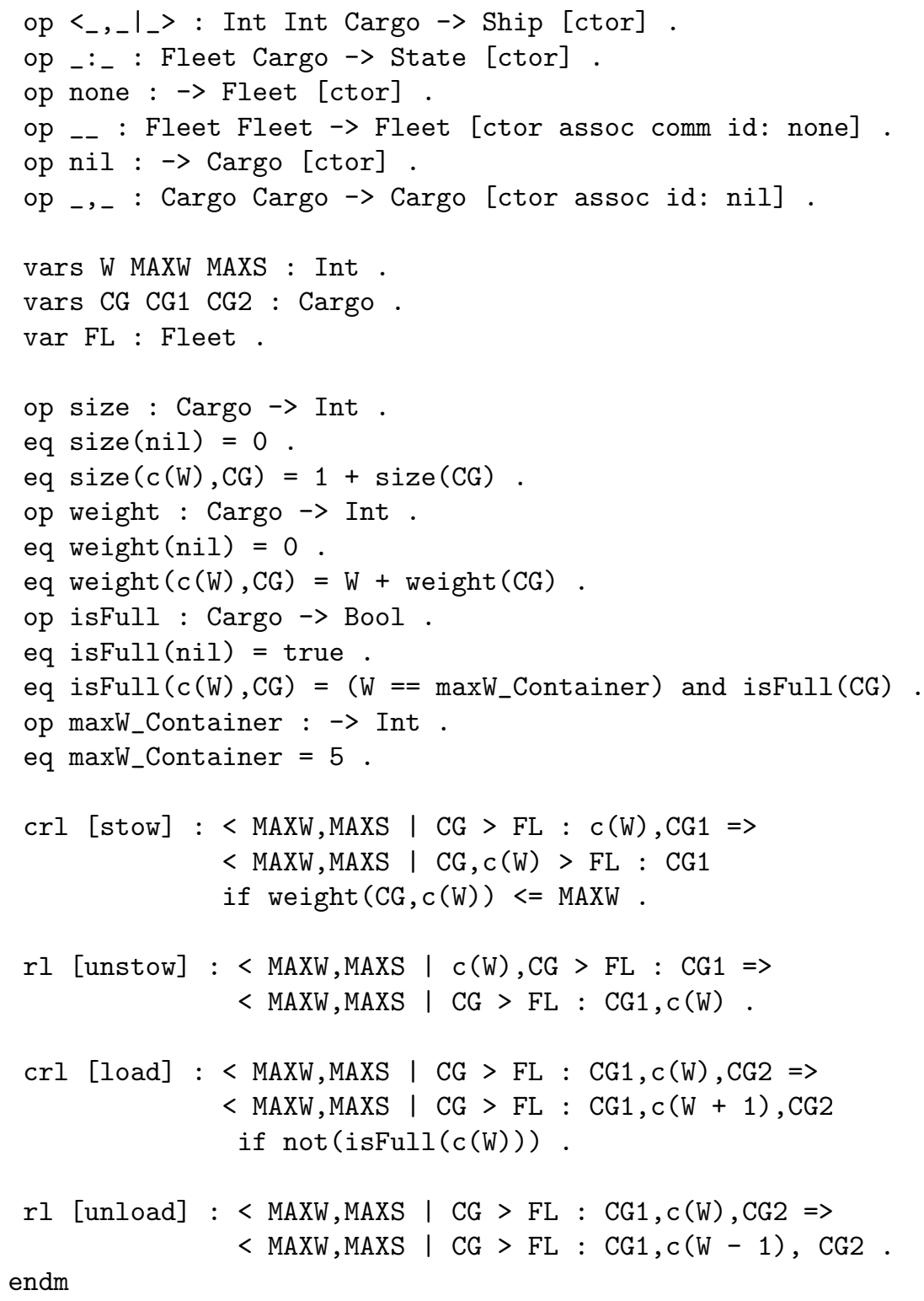

In our specification, system states are modeled by means of terms of sort State with the form FL : CG, where FL is a fleet (i.e., a multiset of ships) and $\mathrm{CG}$ is the cargo at the container terminal ready to be loaded (i.e., a list of containers).

A container of weight $W$ is defined by a term $c(W)$ of sort Container. A collection of containers is built by means of an AU binary operator _, - 
(whose unity or identity element is the constant nil) that basically models a list data structure. Specifically, a list of containers is a term of sort Cargo, whose form is either $c\left(W_{1}\right), c\left(W_{2}\right), \ldots, c\left(W_{n}\right)$ or nil.

Containers are stowed on (resp. unstowed from) ships following a first in, first out strategy. A ship with maximum allowed weight MAXW, maximum allowed capacity (number of containers on board) MAXS, and loaded cargo CG is defined by a term < MAXW, MAXS | CG > of sort Ship. A collection (multiset) of ships is a term of sort Fleet that is built by means of an ACU binary operator _- whose identity element is the constant none. The considered sorts and their associated subsort relations are declared at the top of the CONTAINER-TERMINAL system module (lines 2-4), while the constructor operators, which are required to define the system state structure, are specified by lines $5-11$.

The Maude module also includes some equations that specify the auxiliary functions size (lines 15-17), weight (lines 18-20), and isFull (lines 21-23). The functions size(CG) and weight (CG) respectively return the number of containers and the total weight of the cargo list CG, whereas the Boolean function isFull (CG) checks whether each container in CG is completely filled, with maxW_Container (lines 24-25) being the maximum allowed weight of each container.

The system behavior is specified by means of four rewrite rules. The rule stow (lines 26-28) removes a container from the front of the terminal container list and loads it on an arbitrary ship provided that the total weight of its current cargo plus the weight of the considered container is lower than or equal to MAXW. Likewise, unstow (lines 29-30) removes a container from an arbitrary ship and adds it to the back of the container terminal cargo list. To simplify the model, the rule load (lines 31-33) increases by one unit the weight of an arbitrary container $\mathrm{c}(\mathrm{W})$ located at the cargo-terminal provided $c(W)$ is not already full. Dually, the rule unload (lines 34-35) decreases the weight of $c(W)$ by one unit.

Observe that the considered CONTAINER-TERMINAL system module can produce some awkward, certainly unwanted, system states. For instance, it is possible to reach a system configuration where the current number of loaded containers CG in a ship < MAXW, MAXS | CG > is greater than the allowable ship capacity MAXS. Moreover, there is no lower limit for the weight of a single container, which can lead to loading containers with a negative weight. 


\subsection{Rewriting in Conditional Rewrite Theories}

In a rewrite theory $(\Sigma, E, R)$, with $E=\Delta \cup B$, the concurrent system evolves by rewriting states using equational rewriting, i.e., rewriting with the rewrite rules in $R$ modulo the equations and axioms in $E$ [17].

The Maude interpreter implements equational rewriting by means of two simple relations, namely $\rightarrow_{\Delta, B}$ and $\rightarrow_{R, B}$. These allow rules and equations to be intermixed in the rewriting process by simply using both an algorithm of matching modulo $B^{5}$. Roughly speaking, the relation $\rightarrow_{\Delta, B}$ uses the equations of $\Delta$ (oriented from left to right) as simplification rules. Thus, for any term $t$, by repeatedly applying the equations as simplification rules, we eventually reach a term $t \downarrow_{\Delta, B}$ to which no further equations can be applied. The term $t \downarrow_{\Delta, B}$ is called a canonical form (or irreducible form) of $t$ w.r.t. $\Delta$ modulo $B$. On the other hand, the relation $\rightarrow_{R, B}$ implements rewriting with the rules of $R$, which might be nonterminating and nonconfluent, whereas $\Delta$ is required to be terminating and Church-Rosser (i.e., confluent and sortdecreasing) modulo $B$ [8] in order to guarantee the existence and unicity (modulo $B$ ) of a canonical form w.r.t. $\Delta$ for any term [5].

Formally, $\rightarrow_{R, B}$ and $\rightarrow_{\Delta, B}$ are defined as follows. Given a rewrite rule $[r]:(\lambda \Rightarrow \rho$ if $C) \in R$ (resp., an equation $[e]:(\lambda=\rho$ if $C) \in \Delta)$, a substitution $\sigma$, a term $t$, and a position $w$ of $t, t \stackrel{r, \sigma, w}{\rightarrow}_{R, B} t^{\prime}$ (resp., $t \stackrel{e, \sigma, w}{\rightarrow}_{\Delta, B} t^{\prime}$ ) iff $\lambda \sigma={ }_{B} t_{\mid w}, t^{\prime}=t[\rho \sigma]_{w}$, and $C$ evaluates to true w.r.t $\sigma$. When no confusion arises, we simply write $t \rightarrow_{R, B} t^{\prime}$ (resp. $t \rightarrow_{\Delta, B} t^{\prime}$ ) instead of $t \stackrel{r, \sigma, w}{\rightarrow}_{R, B} t^{\prime}$ (resp. $\left.t \stackrel{e, \sigma, w}{\rightarrow}_{\Delta, B} t^{\prime}\right)$.

Roughly speaking, a conditional rewrite step on the term $t$ applies a rewrite rule/equation to $t$ by replacing a reducible (sub-)expression of $t$ (namely $t_{\mid w}$ ), called the redex, by its contracted version $\rho \sigma$, called the contractum, whenever the condition $C$ is fulfilled.

Note that the evaluation of a condition $C$ is typically a recursive process since it may involve further (conditional) rewrites in order to normalize $C$ to true. Because of this, a conditional equational theory may have a terminating rewrite relation but still exhibit a nontermination behaviour because of a

\footnotetext{
${ }^{5}$ Particularly important instances of $B$-matching occur when $B$ specifies the following combinations of algebraic axioms for an operator op: associative axioms (A), associative and unity axioms (AU), associative and commutative axioms (AC), associative, commutative, and unity axioms (ACU). In the cases when op obeys A or AU (respectively, AC or ACU), any term rooted with op is implicitly handled as a list (respectively, a multiset).
} 
nonterminating "loop" in condition evaluations. In order to guarantee not only that canonical forms exist but also that they can be effectively computed by conditional rewriting, in the following the stronger notion of operational termination in the sense of [15] is considered (that is, terminating in the intuitive sense that an interpreter executing such conditional specifications will terminate for all inputs).

Besides requiring the functional fragment $(\Sigma, \Delta \cup B)$ of $\mathcal{R}=(\Sigma, \Delta \cup B, R)$ to be (operationally) terminating and Church-Rosser, an extra executability condition for equational rewriting in $\mathcal{R}$ is the coherence of the rules in $R$ with the equations in $\Delta$ modulo the axioms in $B$. Altogether, the executability conditions basically ensure that the effect of rewriting in $R$ modulo $\Delta \cup B$ can be achieved by intermingling rewriting with both $R$ and $\Delta$ modulo $B$.

Formally,

Definition 2.2 (coherence) The rewrite theory $\mathcal{R}=(\Sigma, \Delta \cup B, R)$ is coherent if, for each term $t$ to which both an equation of $\Delta$ and a rule of $R$ can be independently applied modulo $B$, the two independent derivations finally meet two terms, $w$ and $w^{\prime}$, such that $w={ }_{B} w^{\prime}$; formally, if $t \rightarrow_{\Delta, B} u$ and $t \rightarrow_{R, B} v$, then there exist $u^{\prime}, u^{\prime \prime}, w$ and $w^{\prime}$ such that the diagram of Figure 1 commutes.

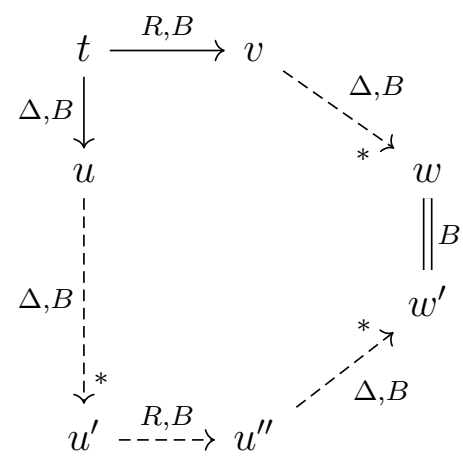

Figure 1: Commutative diagram for the coherence property.

In a coherent rewrite theory (whose equational fragment is (operationally) terminating and Church-Rosser), an equational rewrite step on a term $t$

\footnotetext{
${ }^{6}$ We follow the usual diagrammatic convention, where dashed lines indicate existential quantification.
} 
can be implemented without loss of completeness by applying the following rewrite strategy:

1. Equational simplification of $t$ in $\Delta \operatorname{modulo} B$, that is, reduce $t$ using $\rightarrow_{\Delta, B}$ until the canonical form w.r.t. $\Delta$ modulo $B\left(t \downarrow_{\Delta, B}\right)$ is reached;

2. Rewrite $\left(t \downarrow_{\Delta, B}\right)$ in $R$ modulo $B$ to $t^{\prime}$ using $\rightarrow_{R, B}$, where $t^{\prime} \in[t]_{E}$.

A computation (trace) $\mathcal{C}$ for $t_{0}$ in the conditional rewrite theory $(\Sigma, \Delta \cup$ $B, R$ ) is then deployed as the (possibly infinite) rewrite sequence

$$
t_{0} \rightarrow_{\Delta, B}^{*} t_{0 \downarrow_{\Delta, B}} \rightarrow_{R, B} t_{1} \rightarrow_{\Delta, B}^{*} t_{1 \downarrow} \downarrow_{\Delta, B} \rightarrow_{R, B} \cdots
$$

that interleaves $\rightarrow_{\Delta, B}$ rewrite steps and $\rightarrow_{R, B}$ rewrite steps following the strategy mentioned above. Note that, after each rewrite step using $\rightarrow_{R, B}$, generally the resulting term $t_{i}, i=1, \ldots, n$, is not in canonical form and is thus equationally simplified (or normalized) by using $\rightarrow_{\Delta, B}$ before the subsequent rewrite step using $\rightarrow_{R, B}$ is performed. Also, in the precise strategy adopted by Maude, the last term of a finite computation is finally normalized before the result is delivered. Terms $t_{i}$ (and their canonical forms $t_{i} \downarrow_{\Delta, B}$ ) that appear in a computation are called (system) states, and any sequence $t_{i} \rightarrow_{\Delta, B}^{*} t_{i \downarrow \Delta, B} \rightarrow_{R, B} t_{i+i} \rightarrow_{\Delta, B}^{*} t_{i+i \downarrow \Delta, B}$ from $t_{i}$ to the canonical form of $t_{i+i}$ is called a computation step for $t_{i}$. Therefore, any computation in $\mathcal{R}$ can be conveniently interpreted as a sequence of juxtaposed computation steps $t_{0} \rightarrow_{\mathcal{R}} t_{1} \rightarrow_{\mathcal{R}} t_{2} \rightarrow_{\mathcal{R}}, \ldots$ where each $t_{i}$, with $i>0$, is a canonical form.

\section{Example 2.3}

Let $\mathcal{R}=(\Sigma, \Delta \cup B, R)$ be the rewrite theory in CONTAINER-TERMINAL, the Maude module of Example 2.1. Then,

$$
\begin{aligned}
<5,3 \mid \mathrm{nil}>: \mathrm{c}(1), \mathrm{c}(3) & \rightarrow_{R, B}<5,3 \mid \mathrm{nil}>: \mathrm{c}(1+1), \mathrm{c}(3) \\
& \rightarrow_{\Delta, B}^{*}<5,3 \mid \mathrm{nil}>: \mathrm{c}(2), \mathrm{c}(3)
\end{aligned}
$$

is a computation step in $\mathcal{R}$ that first rewrites the initial state by applying the rule load and then simplifies the resulting state by using the built-in definition for natural addition.

Effective methods for checking the coherence of a conditional order-sorted rewrite theory $\mathcal{R}=(\Sigma, \Delta \cup B, R)$ are formalized in [8] that apply under the following conditions, which we assume for all theories in this paper: 
1. The equational theory $(\Sigma, \Delta \cup B)$ included in $\mathcal{R}$

- is Church-Rosser (modulo B) and operationally terminating; and

- it models an admissible Maude functional module in the sense of [6, Section 4.6], ensuring that all the extra variables in the conditional part of equations will become instantiated by matching in a rewrite step;

2. The rewrite rules in $R$ do not contain rewrite expressions in their conditional part, and are $B$-coherent.

In the following section, we briefly recall the assertion language of [3] that allows formal properties of software systems to be specified in rewriting logic.

\section{The System Assertion Language}

Given the rewrite theory $\mathcal{R}=(\Sigma, \Delta \cup B, R)$, we introduce an ordersorted assertion language $\mathcal{L}$ whose first-order formulas define properties of the system states of $\mathcal{R}$. To simplify our formulation, we assume that the formulas of $\mathcal{L}$ are built over a set of user-defined Boolean operators (predicates) of $\Sigma$ whose semantics is specified by some equations in $\Delta$. The truth values of $\mathcal{L}$ are given by the formulas true and false, and the usual conjunction (and), disjunction (or), exclusive or (xor), negation (not), and implication (implies) logic operators are used to express composite properties. Variables in the formulas are not quantified.

Within this logic framework, we define system assertions (assertions for short) as constrained terms [21]. Formally, a system assertion is a pair $\Pi \mid \varphi$, where $\varphi$ is a quantifier-free Boolean formula that is specialized to a (typically non-ground) term $\Pi$ in $\mathcal{T}(\Sigma, \mathcal{V})$, with $\mathcal{V} a r(\varphi) \subseteq \mathcal{V}$ ar $(\Pi)$.

The reason for avoiding an explicit handling of quantifiers in the definition of $\varphi$ is the need for keeping reasoning complexity manageable while ensuring correctness and completeness of the transformation. For instance, if existential quantification were allowed in $\varphi$, the more costly mechanism of equational narrowing would be required to prove the system assertion $\Pi \mid \varphi$, while some form of "finitization" or "concretization" transformation would be required to deal with universal quantification. Nevertheless, since our assertion language works in tandem with a rewrite theory that allows Boolean functions to be defined and then invoked in $\varphi$, system assertions can characterize a wide spectrum of system states. This is done by defining pure declarative representations of states by means of state templates, 
and invariants that can predicate on the whole system structure or just on fragments of it. Actually, when several distinct, top-level state constructors are declared, we can define several assertions, one for each constructor, in order to capture all of the possible states. Also, for the case when states are made of lists or sets, our assertion language is expressive enough to relate (e.g., count the number of) elements in the list/set. For instance, consider a Maude system module that represents states as lists/sets of elements of the form $\left\langle a_{1}, a_{2}, a_{3}, \ldots, a_{n}\right\rangle$. We can easily define a system assertion that enforces a property $\mathrm{p}$ (A) (only) on states that contain more than 100 elements, as follows:

$$
<A>\mid \operatorname{size}(A)>100 \text { implies } p(A)
$$

where $\mathrm{A}$ is a variable of the (list/set) state sort, size is a user-defined function that counts the elements of a list/set (that can be equationally specified by means of a simple recursion scheme), and $\mathrm{p}(\mathrm{A})$ is a user-defined property over the list/set A.

Operationally, a system assertion $\Pi \mid \varphi$ defines a generic safety property for a state $t$ which specifies a logic invariant $\varphi$ which must be enforced in any subterm of $t$ that is an instance (modulo $\Delta \cup B$ ) of $\Pi$. More formally, a state $t$ satisfies $\Pi \mid \varphi$ (in symbols, $t=\Pi \mid \varphi$ ) iff for every $w \in \mathcal{P}$ os $(t)$ and for every substitution $\sigma$, if $t_{\mid w}={ }_{\Delta \cup B} \Pi \sigma$ then $\varphi \sigma \rightarrow_{\Delta, B}^{*}$ true. The notion of satisfaction can be naturally lifted to sets of assertions: given a set of assertions $\mathcal{A}$, $t$ satisfies $\mathcal{A}$ (in symbols, $t \models \mathcal{A}$ ), iff $t \models a$ for each assertion $a \in \mathcal{A}$. A violation of a system assertion $a$ is detected in a term $t$, whenever $t \not \models a$, that is, when there exist a position $w \in \mathcal{P} o s(t)$ and a substitution $\sigma$ such that $t_{\mid w}={ }_{\Delta \cup B} \Pi \sigma$ and $\varphi \sigma \rightarrow_{\Delta, B}^{*}$ false.

Verification of safety properties amounts to the problem of invariance checking. Hence, we say that a computation $\mathcal{C}$ is safe w.r.t. an assertion set $\mathcal{A}$, if for each state $t$ in $\mathcal{C}, t \models \mathcal{A}$.

\section{Example 3.1}

Let us reinforce safety of the container terminal model of Example 2.1 by specifying some desired properties. Let $\mathcal{A}$ be the assertion set that includes:

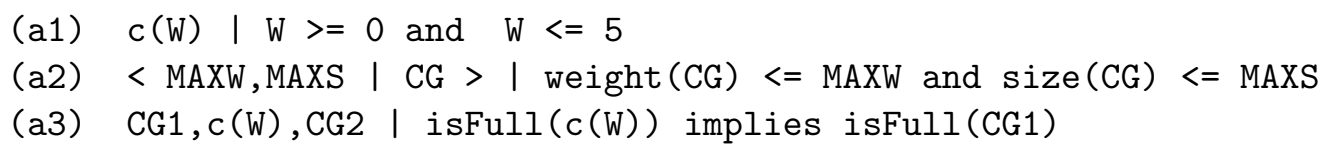


The assertion a1 requires that every container has a weight $W$ ranging from 0 tons to 5 tons independently of its location (ship or container terminal), while the assertion a2 asserts that every ship configuration has a cargo weight and a total number of containers which must not exceed MAXW and MAXS, respectively.

Finally, a3 specifies that, for any container list CG1,c(W), CG2, if the container $\mathrm{c}(\mathrm{W})$ is completely filled, then the containers in the list CG1 are also filled. The goal of this last assertion is to promote container loading over container stowing. In fact, full containers are stowed into a ship before nonfull containers through the stowing fifo strategy encoded by the rule stow.

In the rest of the paper, we present a static correction technique for a rewrite theory $\mathcal{R}=(\Sigma, E, R)$ w.r.t. the assertion set $\mathcal{A}$ that is formalized as a two-phase procedure. First, by using $\mathcal{A}$, the equational theory $(\Sigma, E)$ in $\mathcal{R}$ is augmented with a new set of equations $E^{\prime}$ that identify all possible system assertion violations. Then, the rules of $R$ are transformed into conditional rules by including guarding conditions that invoke the equations of $E^{\prime}$. This guarantees that the application of the transformed rules always produces states that satisfy $\mathcal{A}$, and thus computations in the transformed theory are safe w.r.t. the assertion set $\mathcal{A}$. Clearly, truncating program behaviours of infinite computations could eventually violate liveness and introduce deadlocks. Nevertheless, satisfaction of liveness properties can be eventually analyzed after the correction transformation by means of Maude's model checker [6].

\section{Equational Encoding of System Assertion Violations}

Let $\mathcal{E}=(\Sigma, \Delta \cup B)$ be an equational theory and $\mathcal{A}$ be a set of system assertions that are built using a set of predicates $P$. Without loss of generality, we assume that the equational definition of $P$ is included in $\mathcal{E}$.

We aim to construct an extension of the equational theory $\mathcal{E}$ in which violations of the assertions in $\mathcal{A}$ can be automatically detected. The following definition provides a renaming procedure that is instrumental for the transformation. Roughly speaking, the goal of such a renaming is to neatly separate assertion checking from system computations so that they do not interfere: the overall system execution uses the original operators and equations in the equational theory, while assertion checking is performed on renamed terms. This is not only convenient but it is key to guarantee that the 
correction transformation does not break executability of the original theory by ensuring that confluence, termination, and coherence are preserved.

\section{Definition 4.1 (renaming extension of an equational theory) Let}

$\mathcal{E}=(\Sigma, \Delta \cup B)$ be an equational theory where $(S,<)$ is the sort poset included in $\Sigma$. Let $M \subseteq S$ contain the top sorts of $S, M=\left\{s \in S \mid \nexists s^{\prime} \in\right.$ $S$ such that $\left.s<s^{\prime}\right\}$.

The renaming extension of the equational theory $\mathcal{E}$ is the equational theory $\mathcal{E}^{\prime}=\left(\Sigma \cup \Sigma^{\prime}, \Delta \cup \Delta^{\prime} \cup B\right)$ such that

1. $(S,<)=\left(S^{\prime},<^{\prime}\right)$ where $\left(S^{\prime},<^{\prime}\right)$ is the sort poset included in $\Sigma^{\prime}$ (that is, the renaming extension $\mathcal{E}^{\prime}$ preserves the sort structure of $\left.\mathcal{E}\right)$.

2. $\Sigma^{\prime} \cap \Sigma=\emptyset$, and $\Sigma^{\prime}$ contains one renamed operator $f^{\prime}: s_{1} \ldots s_{n} \rightarrow s$, $n \geq 0$, for each operator $f: s_{1} \ldots s_{n} \rightarrow s$ of $\Sigma$, and $f^{\prime}$ is given the same equational attributes as $f$. Furthermore, $\Sigma^{\prime}$ includes two universal, polymorphic operators $\operatorname{Ren}: \top \rightarrow \top$ and $\operatorname{Ren}^{-1}: \top \rightarrow \top$.

3. $\Delta^{\prime}$ contains the following equations that define the operators Ren and $\operatorname{Ren}^{-1}$, for every possible term in $\mathcal{T}(\Sigma, \mathcal{V})$ and $\mathcal{T}\left(\Sigma^{\prime}, \mathcal{V}\right)$ :

(a) $\operatorname{Ren}\left(f\left(x_{1}, \ldots, x_{n}\right)\right)=f^{\prime}\left(\operatorname{Ren}\left(x_{1}\right), \ldots, \operatorname{Ren}\left(x_{n}\right)\right)$, with $x_{i}: s_{i} \in \mathcal{V}$, $i=1, \ldots, n$, for every function symbol $f: s_{1} \ldots s_{n} \rightarrow s$ of $\Sigma$ with arity $n, n \geq 0$, such that $f$ obeys no unity axiom.

(b) $\operatorname{Ren}^{-1}\left(f^{\prime}\left(x_{1}, \ldots, x_{n}\right)\right)=f\left(\operatorname{Ren}^{-1}\left(x_{1}\right), \ldots, \operatorname{Ren}^{-1}\left(x_{n}\right)\right)$, with $x_{i}: s_{i} \in$ $\mathcal{V}, i=1, \ldots, n$, for every $f^{\prime}: s_{1} \ldots s_{n} \rightarrow s$ of $\Sigma^{\prime}$ such that $f^{\prime} \in \Sigma^{\prime}$ obeys no unity axiom.

(c) For the case of a binary symbol $f: s_{1} s_{2} \rightarrow s$ (and their renamed version $f^{\prime}$ ) with unity element id, the following conditional equations are also contained in $\Delta^{\prime}$ :

$$
\begin{gathered}
\operatorname{Ren}\left(f\left(x_{1}, x_{2}\right)\right)=f^{\prime}\left(\operatorname{Ren}\left(x_{1}\right), \operatorname{Ren}\left(x_{2}\right)\right) \text { if } x_{1}=/=\mathrm{id} \wedge x_{2}=/=\mathrm{id} \\
\operatorname{Ren}^{-1}\left(f^{\prime}\left(x_{1}, x_{2}\right)\right)=f\left(\operatorname{Ren}^{-1}\left(x_{1}\right), \operatorname{Ren}^{-1}\left(x_{2}\right)\right) \text { if } x_{1}=/=\mathrm{id} \wedge \\
x_{2}=/=\mathrm{id}
\end{gathered}
$$

where $x_{1}: s_{1}, x_{2}: s_{2} \in \mathcal{V}$.

(d) $\operatorname{Ren}(x)=x$ [owise] with $x: s \in \mathcal{V}$, for every $s \in M$.

(e) $\operatorname{Ren}^{-1}(x)=x$ [owise] with $x: s \in \mathcal{V}$, for every $s \in M$. 
Note that the extra constraint $x_{1}=/=$ id $\wedge x_{2}=/=$ id of Case (c) is required to avoid nontermination of the renaming process. Indeed, since $t$ is equivalent to $f(t$, id $)$ modulo the unity axiom for $f$, if the condition was omitted, the following nonterminating equational simplification could be delivered for any $t$, where $\operatorname{Ren}(t)$ is simplified infinitely often (and similarly for $\operatorname{Ren}^{-1}\left(t^{\prime}\right)$ for any $\left.t^{\prime}\right)$

$\operatorname{Ren}(t)=_{B} \operatorname{Ren}(f(t$, id $)) \rightarrow_{\Delta^{\prime}, B} f^{\prime}(\operatorname{Ren}(t), \operatorname{Ren}($ id $)) \rightarrow_{\Delta^{\prime}, B} f^{\prime}\left(f^{\prime}(\operatorname{Ren}(t), \operatorname{Ren}(\right.$ id $)), \operatorname{Ren}($ id $\left.)\right) \ldots$

We enforce that terms of the form $\operatorname{Ren}(t)$, with $t \in \mathcal{T}(\Sigma, \mathcal{V})$, are evaluated using an innermost 7 , rewrite strategy that first simplifies the input argument $t$ into the canonical form $t \downarrow_{\Delta, B}$ and then applies the equational definition of Ren to $t \downarrow_{\Delta, B}$. The innermost strategy avoids potential interferences between the renaming and the simplification of a term $t$. In fact, it guarantees that renaming occurs only after term simplification within the original equational theory $(\Sigma, \Delta \cup B)$. More specifically, given a term $t \in \mathcal{T}(\Sigma, \mathcal{V}), \operatorname{Ren}(t)=$ $t^{\prime} \downarrow_{\Delta, B}$ where $t^{\prime} \downarrow_{\Delta, B}$ is a term in $\mathcal{T}\left(\Sigma^{\prime}, \mathcal{V}\right)$ that is computed by replacing each operator $o p$ of the canonical form $t \downarrow_{\Delta, B}$ with $o p^{\prime}$.

Dually, we enforce that $\operatorname{Ren}^{-1}\left(t^{\prime}\right)$ is evaluated by means of an outermost rewrite strategy that simplifies $\operatorname{Ren}^{-1}\left(t^{\prime}\right)$ by applying the equations for the operator $\operatorname{Ren}^{-1}$ at its root position, thereby undoing the renaming of $t^{\prime}$ and recursively restoring the (canonical form of the) original term $t$. The outermost strategy avoids potential interferences between inverse renaming and the assertion-checking equations, as shown in Example 4.4. Furthermore, we also assume that the argument positions of operators Ren and $\operatorname{Ren}^{-1}$ are frozen [8], which implies that rewriting with $R$ is forbidden at those argument positions. This is required to prove the coherence preservation result of Proposition 5.5 in Section 5. In fact, coherence of a conditional rewrite theory might be broken if the Ren operator were applied without the frozenness restriction, as shown in the following example.

\section{Example 4.2}

Consider a rewrite theory $\mathcal{R}=(\Sigma, \Delta \cup B, R)$, where $\Sigma$ contains the operator Ren : $\top \rightarrow \top$ of Definition 4.1 and four constant symbols $a, b, a^{\prime}, b^{\prime}$

\footnotetext{
${ }^{7}$ Innermost (eager) and outermost (lazy) evaluation strategies can be straightforwardly defined in Maude by resorting to the strat operator attribute.
} 
of sort $s$. Also consider that $\Delta$ includes the equational specification of the Ren function of Definition $4.1, B=\emptyset$, and $R=\{a \Rightarrow b\}$. Now, if we assumed that the argument of Ren was not frozen, $\mathcal{R}$ would not be coherent. Indeed, we would have $\operatorname{Ren}(a) \rightarrow_{R, B} \operatorname{Ren}(b) \rightarrow_{\Delta, B} b^{\prime}$ and $\operatorname{Ren}(a) \rightarrow_{\Delta, B}$ a where $b^{\prime} \neq{ }_{B} a^{\prime}$.

However, observe that coherence cannot be broken by renamed terms of the form $\operatorname{Ren}(t)$ under the frozenness restriction of the Ren operator that forbids the application of the rewrite rule $a \Rightarrow b$ on Ren's argument $t$.

Let us now provide an equational representation of the assertion set $\mathcal{A}$, in which we specify a checking equation $e_{a}$ for each assertion $a \in \mathcal{A}$. The goal of $e_{a}$ is to catch every possible violation of $a$ inside any computation state in the rewrite theory $\mathcal{R}$.

Definition 4.3 (assertion-checking equations) Let $\left(\Sigma \cup \Sigma^{\prime}, \Delta \cup \Delta^{\prime} \cup B\right)$ be the renaming extension of the equational theory $\mathcal{E}=(\Sigma, \Delta \cup B)$. Given the system assertion $a=\Pi \mid \varphi$, the assertion-checking equation for $a$, in symbols $e_{a}$, is the conditional equation

$$
\Pi^{\prime}=\text { fail if } \operatorname{not}\left(\operatorname{Ren}^{-1}\left(\varphi^{\prime}\right)\right) .
$$

where $\Pi^{\prime}=\operatorname{Ren}(\Pi), \varphi^{\prime}=\operatorname{Ren}(\varphi)$, and fail is a new, polimorphic, universal constant (not included in $\Sigma \cup \Sigma^{\prime}$ ) of sort $\top$. Given the assertion set $\mathcal{A}$, we define $\mathrm{A}=\left\{e_{a} \mid a \in \mathcal{A}\right\}$.

The idea is now to expand the renaming extension $\mathcal{E}^{\prime}$ of the equational theory $\mathcal{E}$ with $\mathrm{A}$, and to use it to detect any assertion violations at runtime. More specifically, given an assertion $a \in \mathcal{A}$ and a system state st, a single application of $e_{a}$ to a renamed version $\operatorname{Ren}(s t)$ of $s t$ would reduce any subterm of $\operatorname{Ren}(s t)$ that matches $\Pi^{\prime}$ to fail, hence signalling that st violates the assertion $a$.

The renaming operator Ren and its dual counterpart $\operatorname{Ren}^{-1}$ are key in producing a corrected theory whose computations are safe w.r.t. $\mathcal{A}$. Indeed, if we consider an assertion-checking equation $e_{a}=\left(\Pi^{\prime}=\right.$ fail if $\left.\operatorname{not}\left(\operatorname{Ren}^{-1}\left(\varphi^{\prime}\right)\right)\right)$, we can observe the two following facts.

- On the one hand, the application of $\operatorname{Ren}^{-1}$ to the logic formula $\varphi^{\prime}$ ensures that any instance $\varphi \sigma$ of $\varphi$ does not contain renamed terms, and thus $\varphi \sigma$ can be properly simplified to its truth value by using the 
(operationally) terminating and Church-Rosser predicates $P$ that are specified in the original equational theory $\mathcal{E}$.

- On the other hand, the renaming $\Pi^{\prime}$ of $\Pi$ is needed for $e_{a}$ to avoid possible interferences with the equations of the original equational theory $\mathcal{E}$.

\section{Example 4.4}

Consider that the CONTAINER-TERMINAL module of Example 2.1 is augmented by defining a new predicate function empty? that returns true if there is no container in the considered ship, and false otherwise

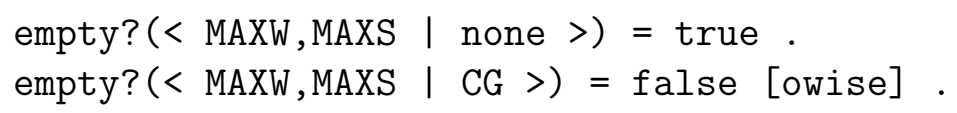

Let the assertion set $\mathcal{A}$ consist of the single assertion

$$
a=<\operatorname{MAXW}, \text { MAXS | CG }>\mid \operatorname{not}(\operatorname{empty} ?(<\operatorname{MAXW}, \text { MAXS } \mid \text { CG }>) \text { ) }
$$

that enforces all possible ship configurations to contain at least one container, and whose associated assertion-checking equation $e_{a}$ is ${ }^{8}$

$$
\begin{aligned}
{\left[e_{a}\right]:<\operatorname{MAXW}, \operatorname{MAXS} } & \mid \mathrm{CG}>^{\prime}=\text { fail } \\
& \text { if } \operatorname{not}\left(\operatorname{Ren}^{-1}\left(\operatorname{not}^{\prime}\left(\operatorname{empty} ?^{\prime}\left(<\operatorname{MAXW}, \text { MAXS } \mid \mathrm{CG}>^{\prime}\right)\right)\right)\right)
\end{aligned}
$$

Note that the left-hand side of $e_{a}$ uses renamed symbols to prevent any interference with the original set of equations of the program. Likewise, the condition is also renamed so that it can be later restored without mixing original and renamed symbols, since the possibly appearing variables are instantiated with renamed terms.

Any attempt to evaluate (an instance of) the condition of $e_{a}$ following an innermost strategy for the Ren ${ }^{-1}$ operator would enter an infinite loop when trying to evaluate the (instance of the) subterm < MAXW, MAXS | CG $>^{\prime}$, since this requires using the equation $e_{a}$ itself once again. On the contrary, by using an outermost strategy, the (instance of the) restored condition not (empty? (< MAXW, MAXS | CG >)) can be safely evaluated by using

\footnotetext{
${ }^{8}$ Note that, in the case of a single mixfix operator such as the Ship constructor $<_{-},\left.{ }_{-}\right|_{-}>$, for simplicity, we just rename one operator symbol, $<_{-},\left.\right|_{-}>^{\prime}$.
} 
the original (operationally terminating and Church-Rosser) set of equations of the program, since it does not match the left-hand side of $e_{a}$ nor any other assertion-based template. Indeed, $\operatorname{Ren}^{-1}$ adopts such an outermost evaluation strategy to prevent its argument to be infinitely unravelled by applying assertion-checking equations.

It is immediate to see that incorporating the set $\mathrm{A}$ of assertion-checking equations into the renaming extension $\mathcal{E}^{\prime}$ of the Church-Rosser and operationally terminating theory $\mathcal{E}$ yields an operationally terminating equational theory. However, the following example shows that this naïvely extended theory might be nonconfluent since more than one irreducible form might exist for a given (renamed) system state when multiple assertion violations are detected within the state.

\section{Example 4.5}

Consider again the CONTAINER-TERMINAL system module of Example 2.1 together with the assertion set $\mathcal{A}$ of Example 3.1, whose associated assertionchecking equations are

$$
\begin{aligned}
& {\left[e_{\mathrm{a} 1}\right]: \mathrm{c}^{\prime}(\mathrm{W})=\text { fail if } \operatorname{not}\left(\operatorname{Ren}^{-1}\left(\mathrm{~W}>=^{\prime} 0^{\prime} \text { and } \mathrm{W}<=^{\prime} 5^{\prime}\right)\right)} \\
& {\left[e_{\mathrm{a} 2}\right]:<\operatorname{MAXW}, \operatorname{MAXS} \mid \mathrm{CG}>^{\prime}=\text { fail }} \\
& \text { if } \operatorname{not}\left(\operatorname { R e n } ^ { - 1 } \left(\text { weight }(C G)<=^{\prime} \text { MAXW and } \operatorname{size}^{\prime}(\mathrm{CG})<=^{\prime}\right.\right. \text { MAXS)) } \\
& {\left[e_{\mathrm{a} 3}\right]: \mathrm{CG} 1,{ }^{\prime} \mathrm{c}^{\prime}(\mathrm{W}),{ }^{\prime} \mathrm{CG} 2=\text { fail }} \\
& \text { if } \operatorname{not}\left(\operatorname { R e n } ^ { - 1 } \left(\text { isFull }^{\prime}\left(c^{\prime}(W)\right)\right.\right. \text { implies' isFull'(CG1)) }
\end{aligned}
$$

Now, observe that confluence can be broken by applying equations $e_{\mathrm{a} 1}$ and $e_{\mathrm{a} 3}$ to the renamed container list

$$
c^{\prime}\left(6^{\prime}\right), c^{\prime}\left(5^{\prime}\right)
$$

Indeed, both assertions a1 and a3 are violated in the given container list. The former is not satisfied because $c^{\prime}\left(6^{\prime}\right)$ exceeds the weight upper limit of 5 tons, while the latter is refuted since there is a full container $c^{\prime}\left(5^{\prime}\right)$ that is preceded in the container list by the overweighted container $c^{\prime}\left(6^{\prime}\right)$ (which actually should be full but not overweighted). Finally, note that the applications of the equations $e_{\mathrm{a} 1}$ and $e_{\mathrm{a} 3}$ to

$$
c^{\prime}\left(6^{\prime}\right), c^{\prime}\left(5^{\prime}\right)
$$


yield two distinct irreducible forms, which are, respectively,

$$
\text { fail, } c^{\prime}\left(5^{\prime}\right) \text { and fail. }
$$

Nevertheless, we are able to recover confluence by providing the transformed theory with additional equations that reduce every (renamed) state that contains a fail subterm to the unique irreducible form fail. Formally,

Definition 4.6 (fail-detecting equations) Let $\left(\Sigma \cup \Sigma^{\prime}, \Delta \cup \Delta^{\prime} \cup B\right)$ be the renaming extension of the equational theory $(\Sigma, \Delta \cup B)$. For every renamed operator $f^{\prime}: s_{1} \ldots s_{n} \rightarrow s \in \Sigma^{\prime}, n \geq 0$, we define the set of fail-detecting equations $F_{f^{\prime}}$ such that

$F_{f^{\prime}}=\left\{\begin{array}{r}\left\{f^{\prime}\left(x_{1}, \ldots, x_{i-1}, \text { fail, } x_{i+1}, \ldots, x_{n}\right)=\text { fail } \mid i=1, \ldots, n\right\} \\ \text { for every } f^{\prime} \in \Sigma^{\prime} \text { without unity, } n \geq 0 \\ \left\{f^{\prime}\left(x_{1}, \text { fail }\right) \text { if } x_{1}=/=\text { fail }\right\} \cup\left\{f^{\prime}\left(\text { fail, } x_{2}\right) \text { if } x_{2}=/=\text { fail }\right\} \\ \text { for every binary operator } f^{\prime} \in \Sigma^{\prime} \text { with unity id }\end{array}\right.$

Furthermore, we define $\mathrm{F}=\bigcup_{f^{\prime} \in \Sigma^{\prime}} F_{f^{\prime}}$.

Similarly to Definition 4.1, Definition 4.6 deals with binary operators with unity in a special way to avoid nontermination of the fail-detecting equations.

Now, we are ready to formalize the $\mathcal{A}$-extension of an equational theory $(\Sigma, \Delta \cup B)$.

Definition 4.7 ( $\mathcal{A}$-extension of $\mathcal{E}$ ) Let $\mathcal{E}=(\Sigma, \Delta \cup B)$ be an equational theory and $\mathcal{A}$ be an assertion set. Let $\mathcal{E}^{\prime}=\left(\Sigma \cup \Sigma^{\prime}, \Delta \cup \Delta^{\prime} \cup B\right)$ be the renaming extension of $\mathcal{E}$. Then, the $\mathcal{A}$-extension of $\mathcal{E}$ is the equational theory $\mathcal{E}^{\mathcal{A}}=\left(\Sigma^{\mathcal{A}}, \Delta^{\mathcal{A}} \cup B\right)$ such that

- $\Sigma^{\mathcal{A}}=\Sigma \cup \Sigma^{\prime} \cup\{$ fail $: \rightarrow \top\}$

- $\Delta^{\mathcal{A}}=\Delta \cup \Delta^{\prime} \cup \mathrm{A} \cup \mathrm{F}$

Note that confluence is not lost by joining $A$ with $F$ because all of the equations in $A \cup F$ have the same right-hand side, fail. 


\section{Example 4.8}

Let $\mathcal{E}=(\Sigma, \Delta \cup B)$ be the equational theory in the CONTAINER-TERMINAL module of Example 2.1 and consider again the assertion set $\mathcal{A}$ of Example 3.1. The $\mathcal{A}$-extension $\left(\Sigma^{\mathcal{A}}, \Delta^{\mathcal{A}} \cup B\right)$ of the equational theory $\mathcal{E}$ is obtained by expanding the renaming extension $\mathcal{E}^{\prime}=\left(\Sigma^{\prime}, \Delta^{\prime} \cup B\right)$ of $\mathcal{E}$ with the assertionchecking equations $e_{\mathrm{a} 1}, e_{\mathrm{a} 2}$, and $e_{\mathrm{a} 3}$ of Example 4.5, and the set of faildetecting equations, which specifically includes

$$
\begin{aligned}
& \mathrm{F}_{-,_{-}^{\prime}}=\left\{\left(\mathrm{X},{ }^{\prime} \text { fail }=\text { fail if } \mathrm{X}=/=\text { nil }\right),\left(\text { fail, }{ }^{\prime} \mathrm{X}=\text { fail if } \mathrm{X}=/=\text { nil }\right)\right\} \\
& \left.\mathrm{F}_{\left.<-,-1\rangle_{-}\right\rangle^{\prime}}=\left\{\left(\langle\text { fail }, \mathrm{X} \mid \mathrm{Y}\rangle^{\prime}=\text { fail }\right),\left(\langle\mathrm{X} \text {, fail } \mid \mathrm{Y}\rangle^{\prime}=\text { fail }\right),(\langle\mathrm{X}, \mathrm{Y}| \text { fail }\rangle^{\prime}=\text { fail }\right)\right\}
\end{aligned}
$$

Consider now a term ship $=<20,3 \mid$ c(8), c(9) $>$ which raises two violations of the assertion $\mathrm{a} 1 \in \mathcal{A}$, since both $\mathrm{c}(8)$ and $\mathrm{c}(9)$ are overweighted. These violations are captured by the following equational simplifications that reduce Ren(ship) to the unique irreducible form fail by using the assertionchecking equation $e_{\mathrm{a} 1}$ as well as the fail-detecting equations in $\mathrm{F}_{-,}$, and $\mathrm{F}_{<-,-1>^{\prime}} \cdot$

$$
\begin{aligned}
\operatorname{Ren}(\operatorname{ship}) \rightarrow_{\Delta^{\mathcal{A}}, B}<20^{\prime}, 3^{\prime} \quad \mid \text { fail },^{\prime} \mathrm{c}^{\prime}\left(9^{\prime}\right)>^{\prime} & \rightarrow_{\Delta^{\mathcal{A}}, B}<20^{\prime}, 3^{\prime} \mid \text { fail }>^{\prime} \\
& \rightarrow_{\Delta^{\mathcal{A}}, B} \text { fail } \\
\operatorname{Ren}(\operatorname{ship}) \rightarrow_{\Delta^{\mathcal{A}}, B}<20^{\prime}, 3^{\prime} \quad \mid \mathrm{c}^{\prime}\left(8^{\prime}\right),^{\prime} \text { fail }>^{\prime} & \rightarrow_{\Delta^{\mathcal{A}}, B}<20^{\prime}, 3^{\prime} \mid \text { fail }>^{\prime} \\
& \rightarrow_{\Delta^{\mathcal{A}}, B} \text { fail }
\end{aligned}
$$

As stated in this section, operational termination and Church-Rosser properties of an equational theory are preserved by the $\mathcal{A}$-extension.

\section{Proposition 4.9 (operational termination preservation)}

Let $\mathcal{E}=(\Sigma, \Delta \cup B)$ be an operationally terminating (modulo B) equational theory and $\mathcal{A}$ be an assertion set. Then, the $\mathcal{A}$-extension of $\mathcal{E}$ is operationally terminating (modulo $B$ ).

Proposition 4.10 (Church-Rosser preservation) Let $\mathcal{E}=(\Sigma, \Delta \cup B)$ be an operationally terminating and Church-Rosser (modulo B) equational theory and $\mathcal{A}$ be an assertion set. Then, the $\mathcal{A}$-extension of $\mathcal{E}$ is ChurchRosser (modulo B).

Given an equational theory $\mathcal{E}$ and an assertion set $\mathcal{A}$, the next result formally states that any violation of $\mathcal{A}$ within the canonical form of a term $t$ can be captured by evaluating $\operatorname{Ren}(t)$ in the $\mathcal{A}$-extension of $\mathcal{E}$. 
Proposition 4.11 (completeness of the $\mathcal{A}$-extension) Let $\mathcal{E}$ be an equational theory. Let $\mathcal{A}$ be an assertion set, and $\mathcal{E}^{\mathcal{A}}=\left(\Sigma^{\mathcal{A}}, \Delta^{\mathcal{A}} \cup B\right)$ be the $\mathcal{A}$-extension of $\mathcal{E}$. Let $a \in \mathcal{A}$ and st $\in \mathcal{T}(\Sigma, \mathcal{V})$. If st $\downarrow_{\Delta, B} \mid \neq a$, then $\operatorname{Ren}(s t) \rightarrow_{\Delta \mathcal{A}, B}^{*}$ fail.

\section{Assertion-driven Correction of Topmost Rewrite Theories}

In this section, we formalize an assertion-driven correction methodology for the class of topmost rewrite theories, that is, theories in which terms can only be rewritten at the root position. This class is of primary importance in the Rewriting Logic framework since a sound and complete procedure exists for goal reachability in topmost theories that has many practical applications (e.g., the analysis of security protocols [19]).

A topmost rewrite theory can be defined as follows [22].

Definition 5.1 (topmost rewrite theory) Let $\mathcal{R}=(\Sigma, E, R)$ be a rewrite theory. Let $S$ be the set of sorts of $\Sigma$. Then, $\mathcal{R}$ is topmost if, for some top sort State $\in S$,

1. for each rule $(\lambda \Rightarrow \rho$ if $C)$ in $R, \lambda$ and $\rho$ are terms of sort State;

2. there is no operator in $\Sigma$ whose arity includes a sort $s$ such that top $(s)=$ State.

The rewrite rules in $R$ are also said to be topmost.

Example 5.2

The CONTAINER-TERMINAL module of Example 2.1 encodes a topmost rewrite theory.

Essentially, our correction technique transforms the rewrite rules of a given topmost rewrite theory $\mathcal{R}$ into guarded, conditional rewrite rules that can only be fired if no system assertion is violated. The transformation builds on the notion of $\mathcal{A}$-extension of $\mathcal{R}$ that we defined in Section 4 .

Before formalizing the theory correction methodology, let us also precisely characterize the notion of correction of a rewrite theory $\mathcal{R}$ w.r.t. an assertion set $\mathcal{A}$.

Definition 5.3 ( $\mathcal{A}$-correction of a rewrite theory) Let $\mathcal{R}$ be a rewrite theory and $\mathcal{A}$ be an assertion set. The rewrite theory $\mathcal{R}^{\prime}$ is a correction of $\mathcal{R}$ w.r.t. $\mathcal{A}$ (in symbols $\mathcal{R}^{\prime} \leq^{\mathcal{A}} \mathcal{R}$ ) if the following requirements hold: 
1. for every rewrite computation $\left(t_{0} \rightarrow_{\mathcal{R}^{\prime}} \ldots \rightarrow_{\mathcal{R}^{\prime}} t_{n}\right)$ in $\mathcal{R}^{\prime}$ s.t. $t_{0}=\mathcal{A}$, a rewrite computation $\left(t_{0} \rightarrow_{\mathcal{R}} \ldots \rightarrow_{\mathcal{R}} t_{n}\right)$ exists in $\mathcal{R}$ and $t_{i} \models \mathcal{A}, i=$ $0, \ldots, n$

2. for every rewrite computation $\left(t_{0} \rightarrow_{\mathcal{R}} \ldots \rightarrow_{\mathcal{R}} t_{n}\right)$ in $\mathcal{R}$ s.t. $t_{i}=\mathcal{A}, i=$ $0, \ldots, n$, a rewrite computation $\left(t_{0} \rightarrow_{\mathcal{R}^{\prime}} \ldots \rightarrow_{\mathcal{R}^{\prime}} t_{n}\right)$ exists in $\mathcal{R}^{\prime}$.

Roughly speaking, Definition 5.3 states that (1) every computation in $\mathcal{R}^{\prime}$ whose initial state satisfies $\mathcal{A}$ is a safe computation w.r.t. $\mathcal{A}$ in $\mathcal{R}$, and (2) any safe computation w.r.t. $\mathcal{A}$ in $\mathcal{R}$ is also reproducible in the corrected theory $\mathcal{R}^{\prime}$.

In topmost rewrite theories, all rewrite steps on system states happen at the top of the term. This implies that each rewrite step $t_{1} \stackrel{r, \sigma, w}{\rightarrow} R, B$ rule $r=(\lambda \Rightarrow \rho$ if $C)$, yields a state $t_{2}$ that is an instance of the right-hand side $\rho$ of the applied rule $r$. Therefore, assertion violations in $t_{2}$ can only occur in those terms that have been introduced by the instantiated righthand side $\rho \sigma$. This suggests to us the idea that a correction refinement $\mathcal{R}^{\prime}$ of the topmost rewrite theory $\mathcal{R}$ w.r.t. $\mathcal{A}$ can be synthesized by simply adding an extra constraint $\operatorname{Ren}(\rho)=/=$ fail to the condition $C$ of every rewrite rule $r=(\lambda \Rightarrow \rho$ if $C) \in \mathcal{R}$. Roughly speaking, the extra constraint guarantees that no assertion violation can occur in (any instance of) the right-hand side $\rho$ of $r$ by checking that (the corresponding renamed instances of) $\rho$ cannot be equationally simplified to the constant fail in the $\mathcal{A}$-extension of the equational theory $\mathcal{E}$. This ensures that any state that can be obtained by applying the transformed rules of $\mathcal{R}^{\prime}$ satisfies all of the assertions in $\mathcal{A}$.

Now, we are ready to formalize our correction transformation for topmost rewrite theories w.r.t. an assertional specification $\mathcal{A}$.

Definition 5.4 ( $\mathcal{A}$-extension of $\mathcal{R}$ ) Let $\mathcal{R}=(\Sigma, \Delta \cup B, R)$ be a topmost rewrite theory, $\mathcal{A}$ be an assertion set, and $\left(\Sigma^{\mathcal{A}}, \Delta^{\mathcal{A}} \cup B\right)$ be the $\mathcal{A}$-extension of the equational theory $(\Sigma, \Delta \cup B)$. The $\mathcal{A}$-extension of $\mathcal{R}$ is defined as the rewrite theory $\mathcal{R}^{\prime}=\left(\Sigma^{\mathcal{A}}, \Delta^{\mathcal{A}} \cup B, R^{\mathcal{A}}\right)$, where

$$
R^{\mathcal{A}}=\{\lambda \Rightarrow \rho \text { if } C \wedge \operatorname{Ren}(\rho)=/=\text { fail } \mid(\lambda \Rightarrow \rho \text { if } C) \in R\} .
$$

The correction transformation in 5.4 preserves the coherence property, when applied to input topmost rewrite theories that satisfy the sufficient conditions of [8, Theorem 5] for enforcing coherence in conditional, ordersorted, rewrite theories. 
Proposition 5.5 (coherence preservation) Let $\mathcal{R}=(\Sigma, \Delta \cup B, R)$ be a topmost rewrite theory that meets the sufficient conditions for coherence modulo $B$ of [8, Theorem 5], and $\mathcal{A}$ be an assertion set. Then, the $\mathcal{A}$-extension of $\mathcal{R}$ is coherent.

Finally, the following result states the soundness of the topmost correction transformation. Even if corrections can change the control flow of the program, they do not introduce new states because of their equational definition. Hence the traces $\left(t_{0} \rightarrow_{\mathcal{R}} \ldots \rightarrow_{\mathcal{R}} t_{n}\right)$ in $\mathcal{R}$ and $\left(t_{0} \rightarrow_{\mathcal{R}^{\prime}} \ldots \rightarrow_{\mathcal{R}^{\prime}} t_{n}\right)$ in $\mathcal{R}^{\prime}$ specify the same state sequence.

\section{Theorem 5.6 (sound topmost correction)}

Let $\mathcal{R}=(\Sigma, \Delta \cup B, R)$ be a topmost rewrite theory, $\mathcal{A}$ be an assertion set, and $\mathcal{R}^{\prime}$ be the $\mathcal{A}$-extension of $\mathcal{R}$ formalized in Definition 5.4. Then $\mathcal{R}^{\prime} \leq^{\mathcal{A}} \mathcal{R}$.

\section{Example 5.7}

Consider the topmost rewrite theory $\mathcal{R}=(\Sigma, \Delta \cup B, R)$ encoded by the CARGO system module of Example 2.1, and the assertion set $\mathcal{A}$ of Example 3.1. Then the $\mathcal{A}$-extension of $\mathcal{R}$ w.r.t. $\mathcal{A}$ is the rewrite theory $\mathcal{R}^{\prime}=\left(\Sigma^{\mathcal{A}}, \Delta^{\mathcal{A}} \cup\right.$ $\left.B, R^{\mathcal{A}}\right)$ where

- $\left(\Sigma^{\mathcal{A}}, \Delta^{\mathcal{A}} \cup B\right)$ is the $\mathcal{A}$-extension of $(\Sigma, \Delta \cup B)$ that has been computed in Example 4.8;

- $R^{\mathcal{A}}$ is the set that contains the following rewrite rules

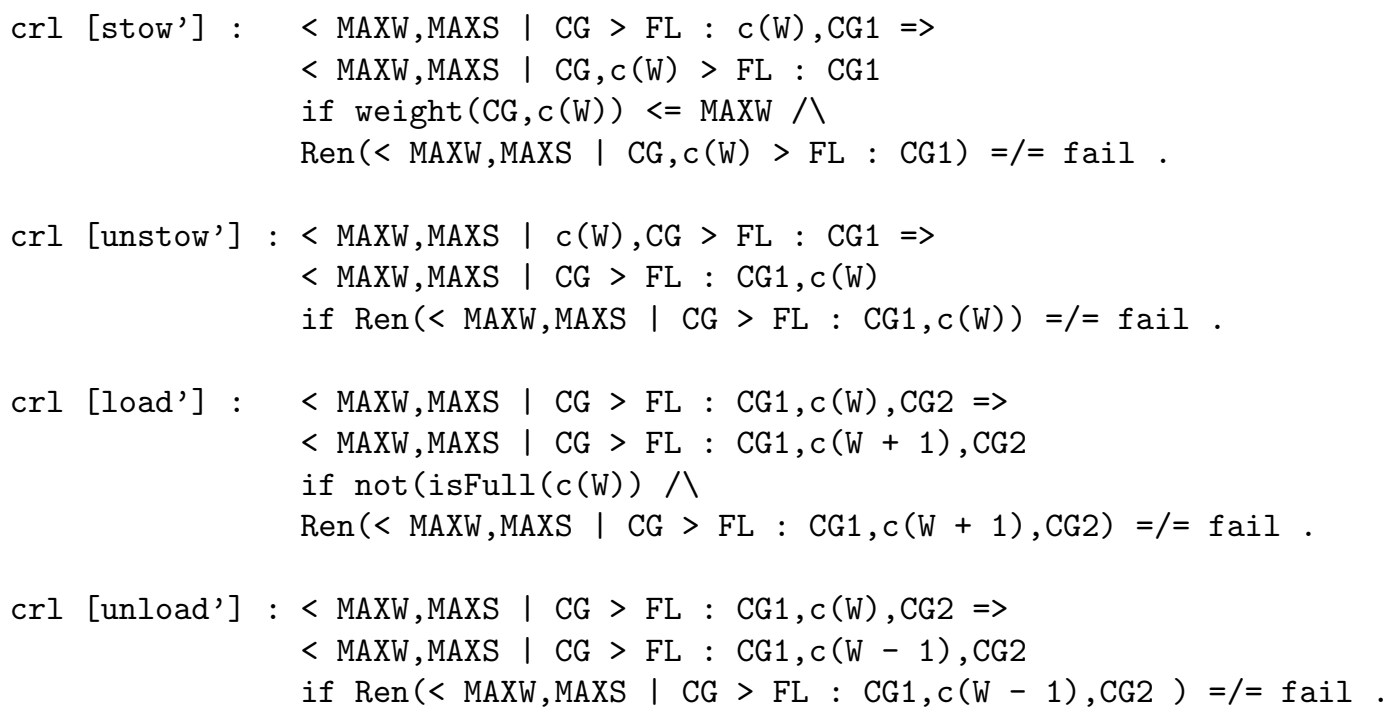


By Theorem 5.6. $\mathcal{R}^{\prime} \leq^{\mathcal{A}} \mathcal{R}$, which implies that $\mathcal{R}^{\prime}$ reproduces all and only the computations of $\mathcal{R}$ that are safe w.r.t. $\mathcal{A}$. For instance, the following computation step

$$
<10,3\left|\mathrm{c}(5)>: \mathrm{c}(0) \rightarrow_{\mathcal{R}}<10,3\right| \mathrm{c}(5)>: \mathrm{c}(-1)
$$

can be given in $\mathcal{R}$ by applying the unload rule, thereby yielding a resulting state $t_{\text {err }}=\langle 10,3 \mid \mathrm{c}(5)\rangle: \mathrm{c}(-1)$ that violates the assertion a1.

Now, observe that there is no way to reach the state $t_{\text {err }}$ from the initial system state $t_{i}=\langle 10,3 \mid \mathrm{c}(5)\rangle: \mathrm{c}(0)>$ in $\mathcal{R}^{\prime}$ because the transformed rule unload' cannot be applied to $t_{i}$. In fact, the instantiated guard

$$
\operatorname{ren}(<10,3 \mid c(5)>: c(0-1))=/=\text { fail }
$$

in the conditional part of unload' evaluates to false and thus prevents the rule unload' from being fired.

\section{Assertion-driven Correction for more Complex Rewrite Theo- ries}

In this section, we enlarge the class of rewrite theories that can be automatically corrected so that no assertion in $\mathcal{A}$ is contravened. This is done by considering two classes of rewrite theories that can be transformed into (semantically equivalent) topmost rewrite theories, and then automatically corrected w.r.t. $\mathcal{A}$ by exploiting Theorem 5.6.

The first class we consider generalizes the topmost modulo $A C U$ rewrite theories of [19] to topmost modulo $A x$ rewrite theories, where $A x$ consists of any of the combinations of axioms ACU, AC, AU, or A for a given binary symbol $\otimes:$ Config Config $\rightarrow$ Config of the signature. The operator $\otimes$ is used to build system configurations that obey the structural axioms of $\otimes$ given by $A x$. The second class we consider formalizes the so-called Russian doll rewrite theories, which allow one to deal with complex, recursively nested state configurations (e.g., multisets of multisets of elements) in which rewrites can happen at any nesting depth of a state.

Topmost modulo $A x$ rewrite theories have many practical applications as they support system configurations that can consist of multisets (defined by the symbol $\otimes$ being a binary ACU/AC operator), or lists (defined by $\otimes$ being $\mathrm{AU} / \mathrm{A})$. This class of theories is particularly useful in the specification of object-oriented systems involving flat configurations in which the distributed 
state is a (multi-)set or a list of objects and messages [19]. Furthermore, different styles of Petri nets can also be modeled via topmost modulo $A x$ rewrite theories [16].

Note that our formalization of topmost modulo $A x$ rewrite theories excludes the cases when $A x$ contains the combination $\mathrm{CU}$, or simply $\mathrm{C}$. The reason is twofold. On the one hand, an operator $\otimes$ that obeys $\mathrm{CU} / \mathrm{C}$ would model states as recursively, nested commutative pairs of terms. This state structure is tricky and generally of little use since it can be replaced by simpler state structures that exploit the more powerful ACU/AC/AU operators in most practical scenarios. On the other hand, if we just need to model states as flat commutative pairs $t_{1} \otimes t_{2}$ (that is, pairs in which the operator $\otimes$ cannot occur either in $t_{1}$ or $t_{2}$ ), this can be done by defining a topmost rewrite theory in which $\otimes$ is a binary $\mathrm{CU}$ (or $\mathrm{C}$ ) operator with sort $s s \rightarrow$ State. This way, there is no need to apply any program transformation, since all states of the form $t_{1} \otimes t_{2}$ are rewritten at the top level and the correction technique of Section 5 can be directly applied.

\subsection{Topmost modulo Ax Rewrite Theories}

Topmost modulo $A x$ rewrite theories can be formalized as follows. We denote by $\alpha(f)$ the set $\alpha(f)=\{A C U, A C, A U, A\}$ of combinations of associativity, commutativity, and/or unity axioms for the binary operator $f \in \Sigma$.

Definition 6.1 (topmost modulo $A x$ rewrite theory) Let $\mathcal{R}=(\Sigma, \Delta \cup$ $B, R)$ be a rewrite theory with a finite poset of sorts $(S,<)$. Let Config be a top sort in $S$, and $\otimes:$ Config Config $\rightarrow$ Config $\in \Sigma$ be a binary operator that obeys a combination of associativity, commutativity, and/or identity axioms $A x \in \alpha(\otimes) \subseteq B$.

The theory $\mathcal{R}$ is said to be topmost modulo $A x$ if

1. for each rule $(\lambda \Rightarrow \rho$ if $C)$ in $R, \lambda$ and $\rho$ are terms of sort Config;

2. $\otimes$ is the only operator in $\Sigma$ whose arity includes a sort $s$ such that $\operatorname{top}(s)=$ Config.

The rewrite rules in $R$ are also said to be topmost modulo $A x$.

Unlike topmost theories that globally rewrite a state at each rewrite step; topmost modulo $A x$ theories allow rewrite rules to be applied to system configuration fragments - thereby implementing local state changes and providing more flexibility and conciseness in theory specification. 


\section{Example 6.2}

Consider the following Maude code fragment that specifies the FlatCargo data structure as a (flat) multiset of containers $c\left(W_{1}\right), c\left(W_{2}\right), \ldots, c\left(W_{n}\right)$.

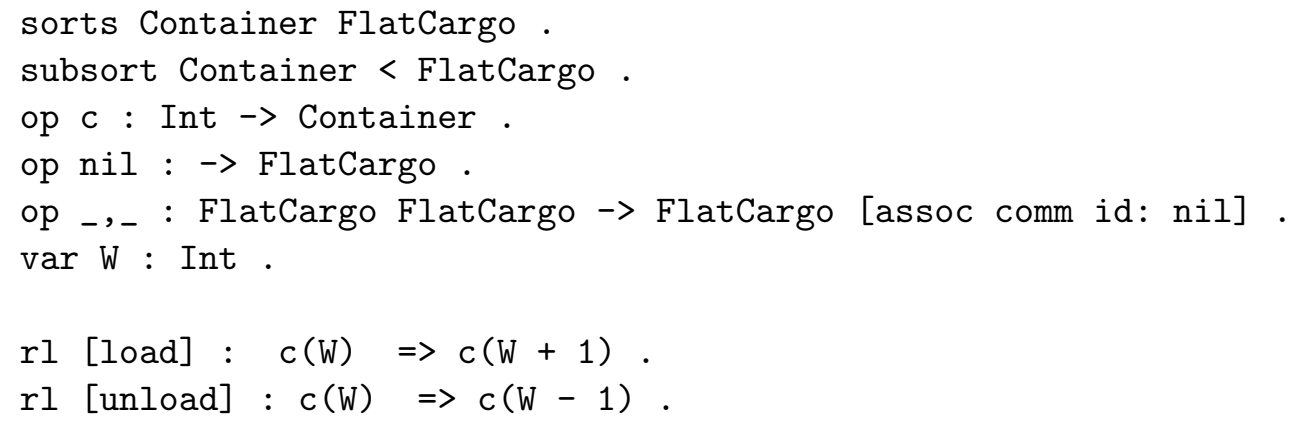

It is immediate to see that this code fragment encodes a topmost modulo ACU rewrite theory. It suffices to interpret the sort FlatCargo as the sort Config of Definition 6.1, and the ACU operator

op _, _ : FlatCargo FlatCargo $\rightarrow$ FlatCargo [assoc comm id: nil]

as the operator $\otimes:$ Config Config $\rightarrow$ Config. Note that the rule load (respectively, unload) allows the weight of an arbitrary container to be locally increased (respectively, decreased) within a cargo configuration $c\left(W_{1}\right), \ldots, c\left(W_{n}\right)$.

Unfortunately, our correction technique cannot be directly applied to this class of rewrite theories because the assertion checking mechanism, which is encoded in the corrected rewrite rules, could fail to catch some assertion violations when local state changes are performed. This is because such rules only check assertions within the rule contractum while ignoring the rest of the configuration (that is, the context at which the replacement takes place). Let us see an example.

\section{Example 6.3}

Consider again the Maude code fragment of Example 6.2 and an assertion set $\mathcal{A}$ that consists of a single assertion that enforces the containers in every system configuration to be pairwise distinct:

$$
c(\mathrm{~W} 1), \mathrm{c}(\mathrm{W} 2) \quad \mathrm{W} 1=/=\mathrm{W} 2
$$


Now, if we apply the correction technique of Section 5 , the following assertionchecking equation is synthesized

$$
c^{\prime}(\mathrm{W} 1), c^{\prime}(\mathrm{W} 2)=\text { fail if } \operatorname{not}\left(\operatorname{Ren}^{-1}(\mathrm{~W} 1=/=, \text { W2 })\right)
$$

and the load rewrite rule of Example 6.2 is refined into the conditional rule

$$
\text { crl [load']: } \quad c(W) \Rightarrow c(W+1) \text { if } \operatorname{Ren}(c(W+1))=/=\text { fail. }
$$

that only increments the container weight provided the condition

$$
\operatorname{Ren}(c(W+1))=/=\text { fail }
$$

is satisfied.

Unfortunately, including Condition (4) into the definition of load' is not enough for the transformed theory to be correct w.r.t. $\mathcal{A}$. In fact, in such a naïvely transformed theory, the following undesired rewrite step can be given by applying load'

$$
c(0), c(1), c(2) \stackrel{\text { load' }}{\rightarrow} c(0+1), c(1), c(2)
$$

that is further simplified into the canonical form $c(1), c(1), c(2)$ which violates Assertion (1). This happens because Assertion (1) is only locally checked over (a renamed version of) the contractum $c(0+1)$ and not against the whole system configuration $c(0+1), c(1), c(2)$.

The applicability problem revealed by Example 6.2 can be overcome by transforming a rewrite theory $\mathcal{R}$ that is topmost modulo $A x$ into an equivalent topmost theory $\hat{\mathcal{R}}$ to which our correction technique applies. Such a transformation was formerly studied in [19] for the case when only ACU operators are considered. Here we extend it with the combinations AC, AU and $\mathrm{A}$.

Definition 6.4 (topmost extension of $\mathcal{R}$ ) Let $\mathcal{R}=(\Sigma, E, R)$ be a topmost modulo $A x$ rewrite theory, where $E=\Delta \cup B$ and $A x \in B$. Let $X, X_{1}$, and $X_{2}$ be variables of sort Config not occurring in either $R$ or $E$. We define the topmost rewrite theory $\hat{\mathcal{R}}=(\hat{\Sigma}, E, \hat{R})$ where $\hat{\Sigma}$ extends $\Sigma$ by adding a new top sort State, and a new operator $\left\{_{-}\right\}:$Config $\rightarrow$ State; and $\hat{R}$ is obtained by transforming $R$ according to $A x$ as follows.

For each $(\lambda \Rightarrow \rho$ if $C) \in R$ 


$\begin{array}{|ll|}\text { Case } A x=A C U . & (\{X \otimes \lambda\} \Rightarrow\{X \otimes \rho\} \text { if } C) \in \hat{R} ; \\ \text { Case } A x=A C . \quad & (\{X \otimes \lambda\} \Rightarrow\{X \otimes \rho\} \text { if } C) \in \hat{R}, \\ & (\{\lambda\} \Rightarrow\{\rho\} \text { if } C) \in \hat{R} ; \\ \text { Case } A x=A U . \quad & \left(\left\{X_{1} \otimes \lambda \otimes X_{2}\right\} \Rightarrow\left\{X_{1} \otimes \rho \otimes X_{2}\right\} \text { if } C\right) \in \hat{R} ; \\ \text { Case } A x=A . \quad & \left(\left\{X_{1} \otimes \lambda \otimes X_{2}\right\} \Rightarrow\left\{X_{1} \otimes \rho \otimes X_{2}\right\} \text { if } C\right) \in \hat{R}, \\ & \left(\left\{X_{1} \otimes \lambda\right\} \Rightarrow\left\{X_{1} \otimes \rho\right\} \text { if } C\right) \in \hat{R}, \\ & \left(\left\{\lambda \otimes X_{1}\right\} \Rightarrow\left\{\rho \otimes X_{1}\right\} \text { if } C\right) \in \hat{R}, \\ & (\{\lambda\} \Rightarrow\{\rho\} \text { if } C) \in \hat{R} .\end{array}$

We call $\hat{\mathcal{R}}$ the topmost extension of $\mathcal{R}$.

Proposition 6.5 (correctness and completeness of the topmost extension) Let $\mathcal{R}$ be a topmost modulo Ax theory and $\hat{\mathcal{R}}$ be the topmost extension of $\mathcal{R}$. For any term $t_{i}$ and $t_{f}$ of sort Config, $t_{i} \rightarrow_{\mathcal{R}}^{*} t_{f}$ iff $\left\{t_{i}\right\} \rightarrow_{\mathcal{R}}^{*}\left\{t_{f}\right\}$.

\section{Example 6.6}

Consider the topmost modulo ACU rewrite theory $\mathcal{R}$ that is specified by the Maude code fragment of Example 6.2. By computing its topmost extension $\hat{\mathcal{R}}$, the rewrite rules load and unload are transformed into the rules

$$
\begin{aligned}
& r l[\text { load-ACU] : }\{X, c(W)\} \Rightarrow\{X, c(W+1)\} . \\
& r l[u n l o a d-A C U]:\{X, c(W)\} \Rightarrow\{X, c(W-1)\} .
\end{aligned}
$$

Now, the undesired computation of Example 6.2 is mimicked in $\hat{\mathcal{R}}$ by the following computation

$$
\{c(0), c(1), c(2)\} \rightarrow_{\mathcal{R}}^{*}\{c(1), c(1), c(2)\}
$$

in which the load-ACU rule is applied to the initial state $\{c(0), c(1), c(2)\}$ to erroneously increase the weight of container c(0).

By exploiting the program transformation for topmost rewrite theories of Section 5, the assertion-driven correction technique can also be applied to the class of topmost modulo $A x$ rewrite theories, and its correction follows from the correction result for the topmost theories (see Theorem 5.6). 
Corollary 6.7 (sound topmost modulo $A x$ correction)

Let $\mathcal{R}=(\Sigma, \Delta \cup B, R)$ be a topmost modulo Ax rewrite theory, with $A x \in B$. Let $\mathcal{A}$ be an assertion set, $\hat{\mathcal{R}}=(\hat{\Sigma}, \Delta \cup B, \hat{R})$ be the topmost extension of $\mathcal{R}$, and $\left(\hat{\Sigma}^{\mathcal{A}}, \Delta^{\mathcal{A}} \cup B\right)$ be the $\mathcal{A}$-extension of $(\hat{\Sigma}, \Delta \cup B)$.

Let $\hat{\mathcal{R}}^{\prime}=\left(\hat{\Sigma}^{\mathcal{A}}, \Delta^{\mathcal{A}} \cup B, \hat{R}^{\mathcal{A}}\right)$ be a rewrite theory such that

$$
\hat{R}^{\mathcal{A}}=\{\lambda \Rightarrow \rho \text { if } C \wedge \operatorname{Ren}(\rho)=/=\text { fail } \mid(\lambda \Rightarrow \rho \text { if } C) \in \hat{R}\} .
$$

Then $\hat{\mathcal{R}}^{\prime} \leq^{\mathcal{A}} \hat{\mathcal{R}}$.

\section{Example 6.8}

Consider the assertion set $\mathcal{A}=\{\mathrm{c}$ (W1), c(W2) | W1 =/= W2 $\}$ of Example 6.3 and the rewrite theory $\mathcal{R}$ of Example 6.2, together with the topmost extension $\hat{\mathcal{R}}$ of $\mathcal{R}$ given in Example 6.6. Then, $\hat{\mathcal{R}}^{\prime}=\left(\hat{\Sigma}^{\mathcal{A}}, \Delta^{\mathcal{A}} \cup B, \hat{R}^{\mathcal{A}}\right)$ includes the conditional rule

$$
\begin{aligned}
\operatorname{crl}[\text { loadC-ACU'] : }\{\mathrm{X}, \mathrm{c}(\mathrm{W})\} \Rightarrow\{\mathrm{X}, \mathrm{c}(\mathrm{W}+1)\} & \\
& \text { if } \operatorname{Ren}(\{\mathrm{X}, \mathrm{c}(\mathrm{W}+1)\})=/=\text { fail } .
\end{aligned}
$$

Note that the application of [loadC-ACU'] completely replaces a multiset $M$ of containers (that matches $\{\mathrm{X}, \mathrm{c}(\mathrm{W})\}$ ) with a new one $M^{\prime}$ (that matches $\{\mathrm{X}, \mathrm{c}(\mathrm{W}+1)\})$. The rule is fired only if the resulting multiset $M^{\prime}$ satisfies the condition $\operatorname{Ren}(X, c(W+1))=/=$ fail, which is true if no violation of the assertion in $\mathcal{A}$ is detected (that is, the containers in $M^{\prime}$ are pairwise disjoint).

Now, as expected, the erroneous computation of Example 6.6, which violates $\mathcal{A}$, cannot be reproduced in $\hat{\mathcal{R}}^{\prime}$.

It is worth noting that the program transformation above can be easily extended to those rewrite theories that include local as well as global state changes, which are respectively modeled by topmost and topmost modulo $A x$ rewrite rules. In this scenario, given a rewrite theory $\mathcal{R}=(\Sigma, \Delta \cup B, R)$, we are always able to partition the set of rewrite rules $R$ into two disjoint sets $R_{\Lambda}$ and $R_{>\Lambda}$ such that $R_{\Lambda}=\{\lambda \Rightarrow \rho$ if $C \mid \lambda, \rho$ are of sort State $\}$ and $R_{>\Lambda}=\{\lambda \Rightarrow \rho$ if $C \mid \lambda, \rho$ are of sort Config $\}$. The set $R_{\Lambda}$ contains the topmost rewrite rules in $R$ that globally rewrite a state at its root position, while $R_{>\Lambda}$ includes the topmost modulo $A x$ rules that locally rewrite an inner state fragment (that is, a configuration within the state).

Now, $\mathcal{R}$ can be turned into a topmost rewrite theory by reusing the sort State included in $\mathcal{R}$ and simply applying the program transformation of Definition 6.4 to $R_{>\Lambda}$, while leaving $R_{\Lambda}$ unchanged. 


\section{Example 6.9}

Let $\mathcal{R}=\left(\Sigma, \Delta \cup B, R_{\Lambda} \cup R_{>\Lambda}\right)$ be a rewrite theory such that $\Sigma$ includes the operators $\{-\}:$ Config $\rightarrow$ State, a $: \rightarrow$ Config, $c: \rightarrow$ Config, as well as the ACU operator $\otimes:$ Config Config $\rightarrow$ Config, and

$$
\begin{aligned}
R_{\Lambda} & =\left\{\left[\mathrm{r}_{1}\right]:\{\mathrm{a} \otimes \mathrm{X}\} \Rightarrow\{\mathrm{X}\}\right\} \\
R_{>\Lambda} & =\left\{\left[\mathrm{r}_{2}\right]: \mathrm{a} \Rightarrow \mathrm{c}\right\}
\end{aligned}
$$

where $\mathrm{X}$ is a variable of sort Config.

Note that the rule $r_{1} \in R_{\Lambda}$ globally rewrites terms of the form $\left\{t_{1} \otimes \ldots \otimes\right.$ $\left.t_{n}\right\}$ of sort State, while $r_{2} \in R_{\Lambda}$ performs a local state change, that is, it allows a state fragment, namely, the configuration a, to be rewritten into the configuration $\mathrm{c}$.

Now, by applying the transformation of Definition 6.4 to $r_{2}$, we get the topmost rule $\left[r_{3}\right]:\{\mathrm{X} \otimes \mathrm{a}\} \Rightarrow\{\mathrm{X} \otimes \mathrm{c}\}$, where $\mathrm{X}$ is a variable of sort Config, and the rewrite theory $\left(\Sigma, \Delta \cup B,\left\{\mathrm{r}_{1}, \mathrm{r}_{3}\right\}\right)$ is thus a topmost rewrite theory (equivalent to $\mathcal{R}$ ) to which the correction methodology can be applied.

\subsection{Russian Doll Rewrite Theories}

Many systems (e.g., distributed object-based systems) can have a complex state structure in which system configuration components (e.g. objects and messages) can themselves contain nested configurations of components (e.g., subobjects and submessages). Typically, these configurations are specified in Maude by means of a nested and recursive multiset structure.

Unfortunately, the topmost modulo $A x$ rewrite theories of Section 6.1 can only deal with flat configurations, and thus cannot be used to model rewrite theories whose states have an inherently nested structure.

The class of Russian doll rewrite theories, originally introduced in [18], generalizes the class of topmost modulo ACU rewrite theories, and precisely captures the nature of recursively nested state structures. Roughly speaking, in a Russian doll rewrite theory, the nested state structuring is specified by a boundary operator ${ }^{9}$ of the form $b: s_{1} \ldots s_{n}$ Config $\rightarrow$ Config, $n \geq 0$, which allows a configuration of sort Config to be encapsulated in a well-delimited

\footnotetext{
${ }^{9}$ To keep the exposition simple, here we consider a single boundary operator $b$. However, as described in [18, multiple boundary operators of the form $b_{j}: \overrightarrow{s_{j}}$ Config $\rightarrow$ Config, $j=$ $1, \ldots, m$ could be specified, each of which has distinct argument sorts $\overrightarrow{s_{j}}$.
} 
structure. This structure may also include additional parameters of sorts $s_{1}, \ldots, s_{n}$ that may be convenient to better describe system configurations. Then, a state st can contain several nested configurations, each of which is wrapped by means of the boundary operator $b$.

The assertion-driven program correction technique of previous sections cannot be directly applied to Russian doll rewrite theories since rewrites in a state can happen at any level of nesting. Nonetheless, analogously to the case of topmost modulo $A x$ theories, we can transform Russian doll theories into equivalent, topmost theories for which corrections w.r.t. $\mathcal{A}$ can be computed. This is essentially done by adapting the program transformation of [19], which is correct under the reasonable assumptions that equations do not change the depth of nesting of configurations and rewrite rules do not increase it.

Following [19], the formalization of the class of the Russian doll theories requires the following auxiliary definitions.

Definition 6.10 (Russian doll signature) Let $\Sigma$ be a signature, whose set of sorts is $S$. We say that $\Sigma$ is a Russian doll signature if

- S includes the sorts Config and FlatConfig, with FlatConfig < Config, and Config is a top sort in $S / \equiv_{<}$. Furthermore, for each sort $s \in S$, $s<$ Config implies $s<$ FlatConfig.

- The only operators in $\Sigma$ whose arity includes a sort s such that top $(s)=$ Config are:

$$
\begin{aligned}
\otimes_{-} & : \text {FlatConfig FlatConfig } \rightarrow \text { FlatConfig } \\
\otimes_{-} & : \text {Config Config } \rightarrow \text { Config } \\
b & : \vec{s} \text { Config } \rightarrow \text { Config }
\end{aligned}
$$

where $\otimes$ obeys the algebraic axioms $A C U$. Furthermore, for each operator $f: \vec{w} \rightarrow$ Config $\in \Sigma, f$ is either $\otimes$ or $b$.

Definition 6.11 (nesting depth) Given a Russian doll signature $\Sigma$, a term $t \in \mathcal{T}(\Sigma, \mathcal{V})$ is of bounded nesting if, for all $x \in \mathcal{V}$ ar $(t), x$ is of sort Config implies $x$ is of sort FlatConfig.

Given a term $t$ of bounded nesting, we define the nesting depth of $t$ as 
follows:

$$
\operatorname{depth}(t)= \begin{cases}0 & t \notin \mathcal{T}(\Sigma, \mathcal{V})_{\text {Config }} \text { or } \\ & t \in \mathcal{T}(\Sigma, \mathcal{V})_{\text {FlatConfig }} \\ \max \left(\operatorname{depth}\left(t_{1}\right), \operatorname{depth}\left(t_{2}\right)\right) & t=t_{1} \otimes t_{2} \\ \operatorname{depth}(t)+1 & t=b\left(\vec{p}, t_{1}\right)\end{cases}
$$

Now, a Russian doll rewrite theory is formally defined as follows.

Definition 6.12 (Russian doll rewrite theory) Let $\mathcal{R}=(\Sigma, \Delta \cup B, R)$ be a rewrite theory. Then, $\mathcal{R}$ is a Russian doll rewrite theory if

1. $\Sigma$ is a Russian doll signature;

2. for each equation $(\lambda=\rho$ if $C) \in \Delta$ and substitution $\sigma, \sigma(\lambda)$ is of bounded nesting iff $\sigma(\rho)$ is of bounded nesting, and if $\sigma(\lambda)$ and $\sigma(\rho)$ are of bounded nesting, then depth $(\sigma(\lambda))=\operatorname{depth}(\sigma(\rho))$;

3. for each rewrite rule $(\lambda \Rightarrow \rho$ if $C) \in R, \lambda$ and $\rho$ are of sort Config, and for each substitution $\sigma$ such that $\sigma(\lambda)$ and $\sigma(\rho)$ are of bounded nesting, $\operatorname{depth}(\sigma(\lambda)) \geq \operatorname{depth}(\sigma(\rho))$.

Roughly speaking, Condition 2 in Definition 6.12 ensures that equations do not change the nesting depth of terms, while Condition 3 enforces that rewrites do not increase the nesting depth of terms. Moreover, for Russian doll theories, we have that if $t_{1}$ is a term of bounded nesting, $t_{1} \rightarrow_{\mathcal{R}} t_{2}$ implies that $t_{2}$ is of bounded nesting and $\operatorname{depth}\left(t_{1}\right) \geq \operatorname{depth}\left(t_{2}\right)$.

\section{Example 6.13}

The following fragment of Maude code extends the Cargo data structure of Example 2.1 that models flat lists of containers $c\left(W_{1}\right), c\left(W_{2}\right), \ldots, c\left(W_{n}\right)$ by considering multisets of elements that can be either simple containers $c(W)$ or compound containers. Compound containers are in turn multisets of simple or compound containers.

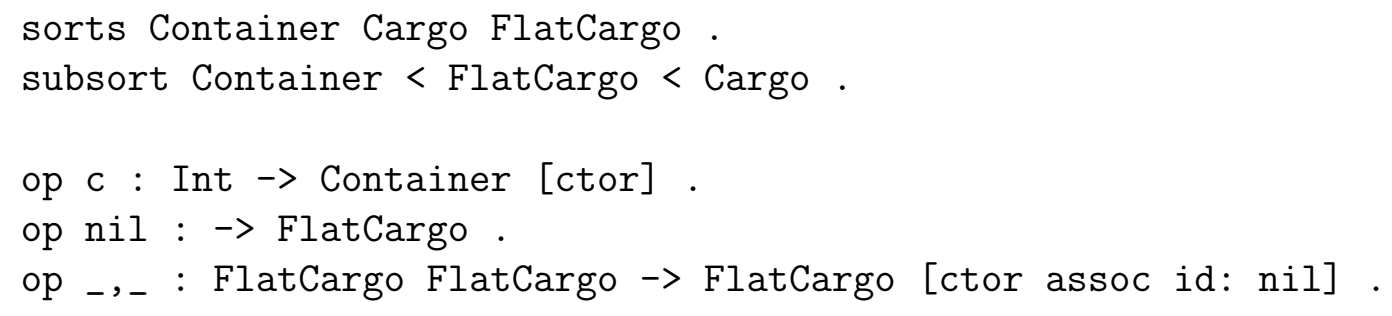




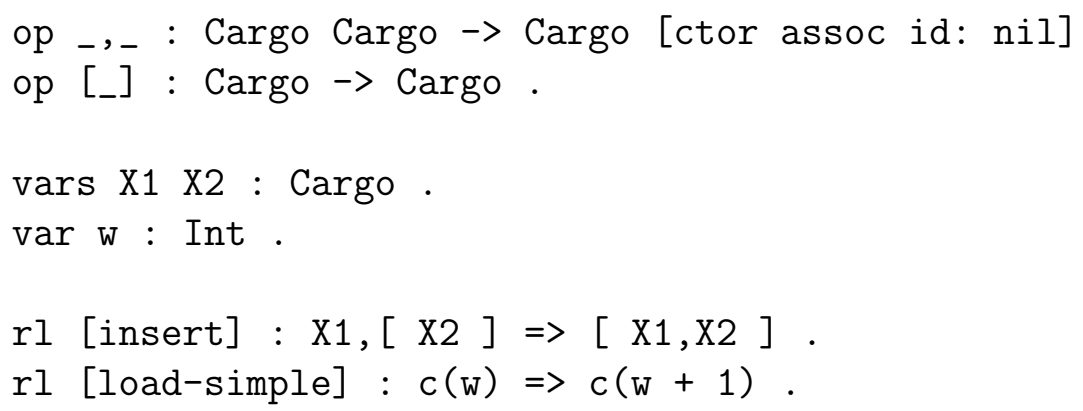

Note that the considered code fragment represents a Russian doll rewrite theory. It suffices to interpret sorts FlatCargo and Cargo as the sorts FlatConfig and Config of Definition 6.12, and the operators

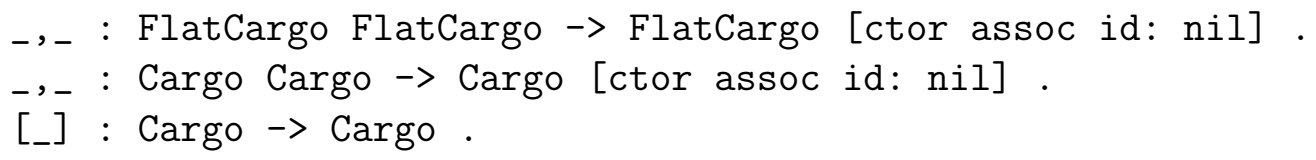

as the operators $\otimes:$ FlatConfig FlatConfig $\rightarrow$ FlatConfig, $\otimes:$ Config Config $\rightarrow$ Config, and $b: \vec{s}$ Config $\rightarrow$ Config, where the sort list $\vec{s}$ is empty. The new Cargo data structure allows multisets of simple and compound containers at distinct nesting levels to be specified. For instance,

$$
c(4),[c(3), c(1)],[[c(4), c(5)], c(3)]
$$

is a depth-2 term of sort Cargo.

The rewrite rules load-simple and insert specify actions for simple and compound containers, respectively. The former increases the weight of a simple container, while the latter allows simple as well as compound containers to be inserted into another compound container.

The next definition is a natural extension of the program transformation of [19] that turns a Russian doll rewrite theory $\mathcal{R}$ into a topmost rewrite theory $\mathcal{R}_{n}$, which is able to deal with configurations whose rule structure has a fixed nesting depth.

Definition 6.14 (topmost $n$-extension of $\mathcal{R}$ ) Let $\mathcal{R}=(\Sigma, \Delta \cup B, R)$ be a Russian doll rewrite theory, and $n$ be a natural number. We define the topmost $n$-extension of $\mathcal{R}$ as the topmost rewrite theory $\mathcal{R}_{n}=\left(\Sigma_{n}, \Delta \cup B, R_{n}\right)$ where 
- $\Sigma_{n}$ extends $\Sigma$ by adding a new top sort State, and a new operator $\{-\}:$ Config $\rightarrow$ State;

- for each rewrite rule $(\lambda \Rightarrow \rho$ if $C) \in R$ and $0 \leq k \leq n$

$$
\begin{aligned}
& \left\{C_{0} \otimes b\left(\vec{x}_{1}, C_{1} \otimes b\left(\vec{x}_{1}, \ldots b\left(\vec{x}_{k}, C_{k} \otimes \lambda\right) \ldots\right)\right)\right\} \Rightarrow \\
& \left\{C_{0} \otimes b\left(\vec{x}_{1}, C_{1} \otimes b\left(\vec{x}_{2}, \ldots b\left(\vec{x}_{k}, C_{k} \otimes \rho\right) \ldots\right)\right)\right\} \text { if } C \in R_{n}
\end{aligned}
$$

where $C_{0}, C_{1}, \ldots, C_{k}$ are variables of sort Config, and $\vec{x}_{1}, \vec{x}_{2} \ldots, \vec{x}_{k}$ are sequences of variables of the sorts required by the boundary operator $b$.

\section{Example 6.15}

Consider the Russian doll rewrite theory encoded by the Maude fragment of Example 6.13. Then, its topmost 1-extension is a topmost rewrite theory that contains the following rewrite rules:

rl [insert-0] : $\{\mathrm{CO}, \mathrm{X} 1,[\mathrm{X} 2]\} \Rightarrow\{\mathrm{CO},[\mathrm{X} 1, \mathrm{X} 2]\}$.

$r 1$ [insert-1] : $\{\mathrm{CO},[\mathrm{C} 1, \mathrm{X} 1,[\mathrm{X} 2]]\} \Rightarrow\{\mathrm{CO},[\mathrm{C} 1,[\mathrm{X} 1, \mathrm{X} 2]]\}$.

$r l[$ load-simple-0] : $\{\mathrm{CO}, \mathrm{C}(\mathrm{w})\} \Rightarrow\{\mathrm{CO}, \mathrm{c}(\mathrm{w}+1)\}$.

rl [load-simple-1] : $\{\mathrm{CO},[\mathrm{C} 1, \mathrm{C}(\mathrm{w})]\} \Rightarrow\{\mathrm{CO},[\mathrm{C} 1, \mathrm{C}(\mathrm{w}+1)]\}$.

It is worth noting that the rewrite rules in the computed 1-extension manage insert and load operations at a nesting depth smaller than or equal to 1. Specifically, the two variants of the original load-simple rule allow to loading simple containers located at depth 0 or 1 within a Cargo structure. Likewise, the rules insert- 0 and insert-1 transfer a multiset of containers $\mathrm{X} 1$ located at depth 0 and 1 , respectively, to a deeper compound container.

Equivalence between a Russian doll rewrite theory and its $n$-extension $\mathcal{R}_{n}$ is given w.r.t. configurations of bounded nesting with a depth equal to $n$, for any natural number $n$ [19]. This means that if $t_{i}$ is a configuration of bounded nesting with $\operatorname{depth}\left(t_{i}\right)=n$ in $\mathcal{R}$, then $t_{i}$ can be rewritten in $t_{f}$ in $\mathcal{R}$ if and only if $\left\{t_{i}\right\}$ can be rewritten in $\left\{t_{f}\right\}$ in $\mathcal{R}_{n}$. More formally,

Proposition $6.16([19])$ Let $\mathcal{R}$ be a Russian doll rewrite theory. Let $t_{i}$ be a term of bounded nesting of sort Config such that depth $\left(t_{i}\right)=n$. Let $\mathcal{R}_{n}$ be the topmost $n$-extension of $\mathcal{R}$. Then, $t_{i} \rightarrow_{\mathcal{R}}^{*} t_{f}$ iff $\left\{t_{i}\right\} \rightarrow_{\mathcal{R}^{n}}^{*}\left\{t_{f}\right\}$. 
Now, since $\mathcal{R}_{n}$ is a topmost rewrite theory, we can directly apply our correction technique and generate sound theory corrections (in the sense of Theorem 5.6) w.r.t. $\mathcal{A}$. Indeed, the following corollary holds.

Corollary 6.17 (sound topmost $n$-extension correction) Let $\mathcal{R}=(\Sigma$, $\Delta \cup B, R)$ be a Russian doll rewrite theory. Let $\mathcal{A}$ be an assertion set, $\mathcal{R}_{n}=$ $\left(\Sigma_{n}, \Delta \cup B, R_{n}\right)$ be the topmost n-extension of $\mathcal{R}$, and $\left(\Sigma_{n}^{\mathcal{A}}, \Delta^{\mathcal{A}} \cup B\right)$ be the $\mathcal{A}$-extension of $\left(\Sigma_{n}, \Delta \cup B\right)$.

Let $\mathcal{R}_{n}^{\prime}=\left(\Sigma_{n}^{\mathcal{A}}, \Delta^{\mathcal{A}} \cup B, R_{n}^{\mathcal{A}}\right)$ be a rewrite theory such that

$$
R_{n}^{\mathcal{A}}=\left\{\lambda \Rightarrow \rho \text { if } C \wedge \operatorname{Ren}(\rho)=/=\text { fail } \mid(\lambda \Rightarrow \rho \text { if } C) \in R_{n}\right\} .
$$

Then $\mathcal{R}_{n}^{\prime} \leq^{\mathcal{A}} \mathcal{R}_{n}$.

In Russian doll theories, configurations are specified by means of the ACU operator $\otimes$ that allows (nested) multisets of elements to be composed together. Actually, it would be possible to consider variants of $\otimes$ that obey distinct combinations of algebraic axioms such as $\mathrm{AC}$, AU, or A, similarly to the case of topmost modulo $A x$ theories of Section 6.1. Nonetheless, this is typically not practical for correction purposes since the number of rewrite rules in the computed topmost $n$-extension $\mathcal{R}_{n}$ could become intractable even for small values of $n$. Indeed, a linear increment of the nesting depth $n$ yields an exponential growth of the number of rewrite rules in $\mathcal{R}_{n}$. Let us see an example.

\section{Example 6.18}

Let $\mathcal{R}$ be a Russian doll rewrite theory that includes the rewrite rule $[r]: a \Rightarrow c$. Further, for the sake of readability, we consider a simple boundary operator []] : Config $\rightarrow$ Config that encapsulates configurations without additional parameters.

Now, by Definition 6.14, the topmost 1-extension $\mathcal{R}_{1}$ of $\mathcal{R}$ contains the following two rules that mimick $r$ within $\mathcal{R}_{1}$ :

$$
\begin{aligned}
& {\left[\mathrm{r}_{1}\right]:\left\{\mathrm{C}_{0} \otimes \mathrm{a}\right\} \Rightarrow\left\{\mathrm{C}_{0} \otimes \mathrm{c}\right\}} \\
& {\left[\mathrm{r}_{2}\right]:\left\{\mathrm{C}_{0} \otimes\left[\mathrm{C}_{1} \otimes \mathrm{a}\right]\right\} \Rightarrow\left\{\mathrm{C}_{0} \otimes\left[\mathrm{C}_{1} \otimes \mathrm{c}\right]\right\}}
\end{aligned}
$$

Now, suppose that $\otimes$ is AC instead of being ACU. In this case, the algebraic axiom $\mathrm{U}$ must be explicitly modeled in the 1-extension $\mathcal{R}_{1}$ by defining distinct rule patterns that consider the presence of both context variables $C_{0}$ and $C_{1}$, 
as well as the absence of one or both context variables. Thus, $\mathcal{R}_{1}$ must contain the following rules:

$$
\begin{aligned}
& {\left[r_{1}\right]:\left\{C_{0} \otimes a\right\} \Rightarrow\left\{C_{0} \otimes c\right\}} \\
& {\left[r_{2}\right]:\left\{C_{0} \otimes\left[C_{1} \otimes a\right]\right\} \Rightarrow\left\{C_{0} \otimes\left[C_{1} \otimes c\right]\right\}} \\
& {\left[r_{3}\right]:\{a\} \Rightarrow\{c\}} \\
& {\left[r_{4}\right]:\{[a]\} \Rightarrow\{[c]\}} \\
& {\left[r_{5}\right]:\left\{C_{0} \otimes[a]\right\} \Rightarrow\left\{C_{0} \otimes[c]\right\}} \\
& {\left[r_{6}\right]:\left\{\left[C_{1} \otimes a\right]\right\} \Rightarrow\left\{\left[C_{1} \otimes c\right]\right\}}
\end{aligned}
$$

Therefore, we need 6 rules in $\mathcal{R}_{1}$ to mimick the behavior of $r$ in the case when $\otimes$ is $\mathrm{AC}$.

\section{Empirical evaluation}

The program correction methodology defined in this paper has been efficiently implemented in a Maude tool called ÁTAME (Assertion-based Theory Amendment in MaudE). The tool has been implemented in Maude itself by using Maude's meta-level capabilities. ÁTAME integrates a RESTful Web service that is written in Java, and an intuitive Web user interface that is based on AJAX technology and is written in HTML5 canvas and Javascript. The implementation contains about 600 lines of Maude source code, 600 lines of $\mathrm{C}++$ code, 750 lines of Java code, and 700 lines of HTML5 and JavaScript code. The correction tool ÁTAME is publicly available together with a number of examples at http://safe-tools.dsic.upv.es/atame.

In this section, we first illustrate the correction for the container terminal model of Section 2 that can be automatically synthesized by using ÁTAME. Then, we summarize the experimental results that we obtain on a set of representative benchmarks.

\section{1. ÁTAME to the Rescue: a Typical Repair Session}

Let us show how ÁTAME works in practice by showing a repair session for our container terminal specification of Example 2.1.

Maude programs can be either uploaded in ÁTAME as simple .maude module files or written from scratch inside a dedicated edit box. A collection of Maude programs, which includes the CONTAINER-TERMINAL module of Example 2.1, is provided with the tool for demonstration purposes. 
To start the repair session, we can just select the CONTAINER-TERMINAL module under examination from the preloaded programs (see Figure 2) and proceed through the next steps.

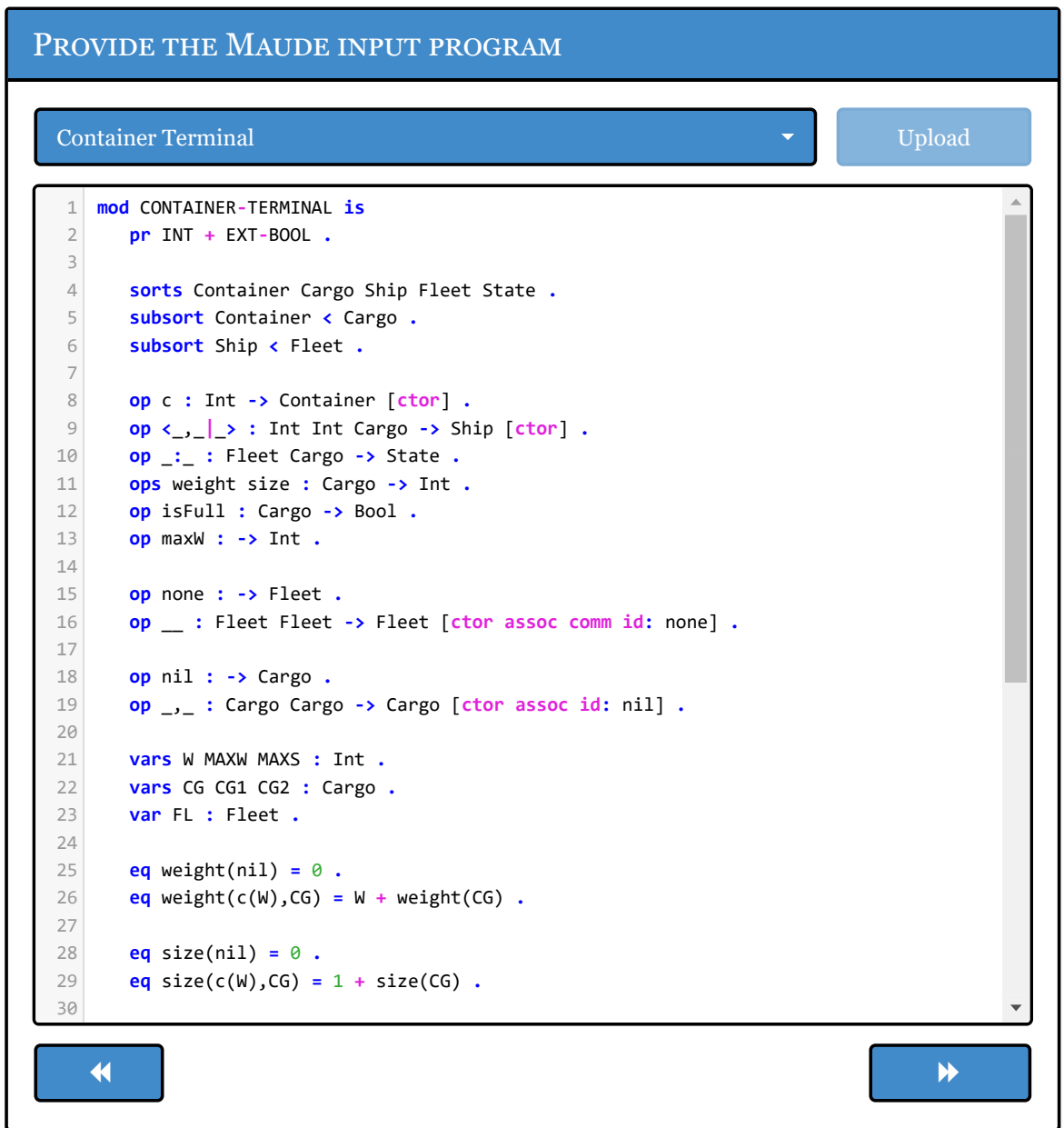

Figure 2: CONTAINER-TERMINAL module loaded in ÁTAME.

The next phase allows the user to specify safety properties to be enforced on the input program. These properties are modeled as system assertions which may use logic predicates that are already defined in the program or new ones that are specified at this stage. Figure 3 illustrates the input phase of the system assertions of Example 3.1 in ÁTAME. Finally, by pressing the Fix Program button, we run our static program repair procedure that 


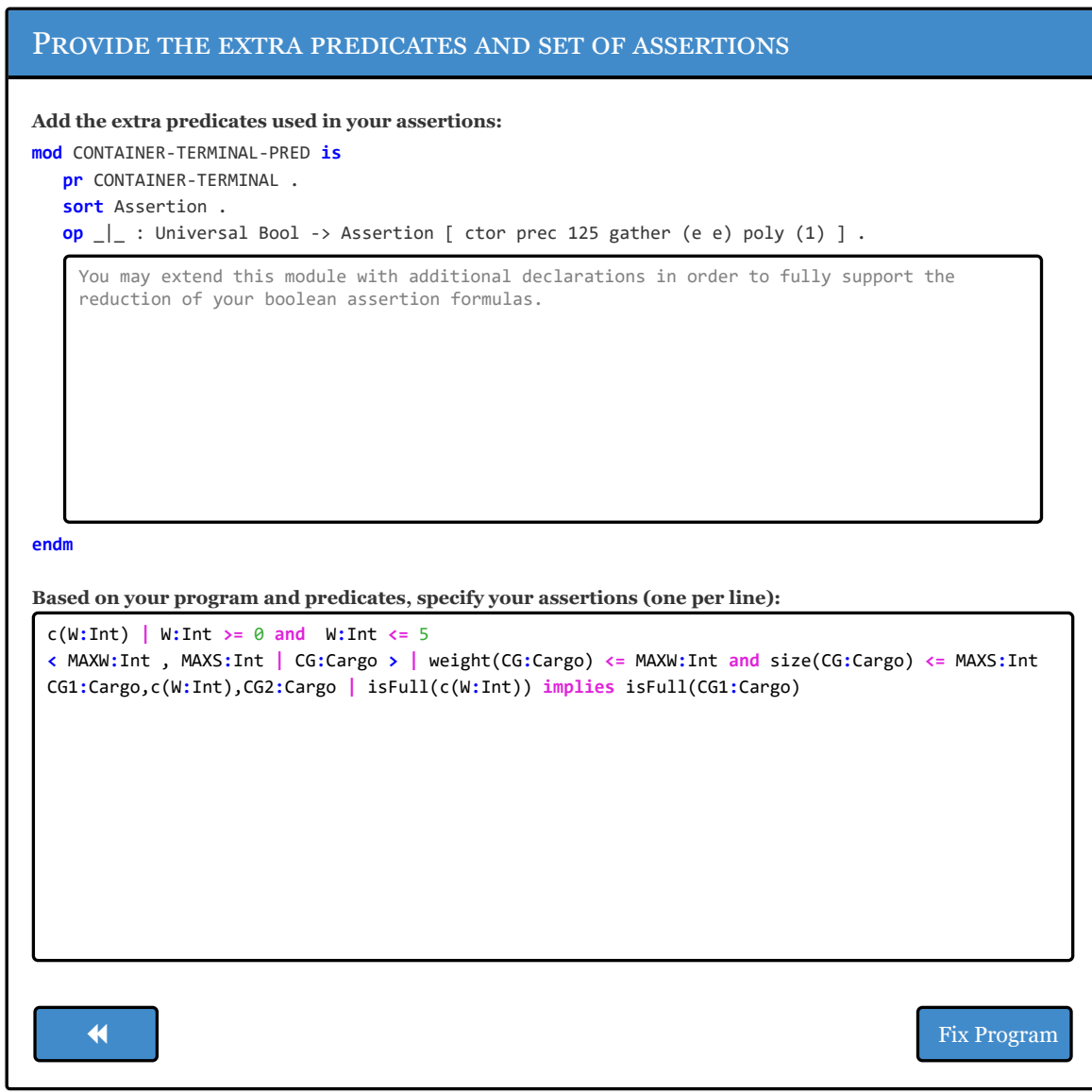

Figure 3: Input of the system assertions of Example 3.1 for the CONTAINER-TERMINAL module.

automatically yields a corrected version of the input program in which all computations are safe w.r.t. the considered assertions. Figure 4 shows (a fragment of) the correction generated for the CONTAINER-TERMINAL module (i.e., the CONTAINER-TERMINAL-FIX module).

As an additional feature, ÁTAME provides the interconnection with the ANIMA Maude stepper [4, which integrates program animation capabilities [1] into ÁTAME. Specifically, by means of the Animate button, the user is allowed to interactively inspect an incrementally generated fragment of the 


\section{FIXED PROGRAM RESULT (INCLUDES PRELUDE IMPORTS)}

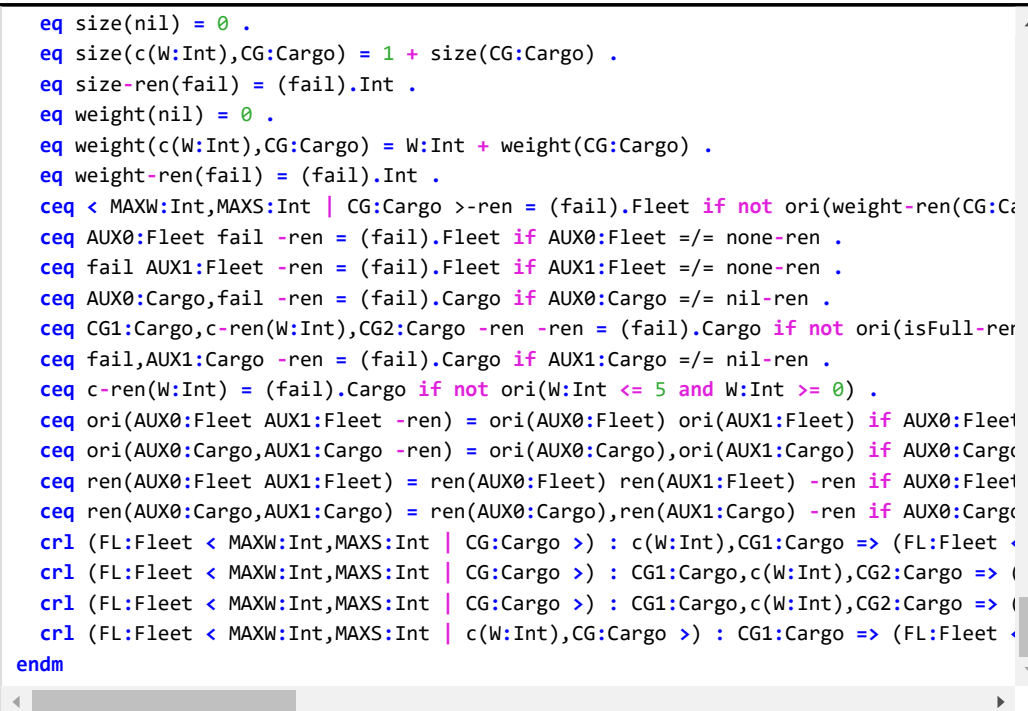

Figure 4: Correction for the CONTAINER-TERMINAL module.

computation tre ${ }^{10}$ of the corrected program for a given initial state.

Figure 5 shows together a fragment of the computation tree of the CONTAINER-TERMINAL module (figure above) and its counterpart in the repaired module CONTAINER-TERMINAL-FIX (figure below). Note that state $s_{39}$ in the computation tree of the original program does not belong to the computation tree of the repaired module. This is correct because $s_{39}$ violates the specified assertion

$$
\text { CG1,c(W), CG2 | isFull(c(W)) implies isFull(CG1) }
$$

and thus it cannot appear in any computation of CONTAINER-TERMINAL-FIX.

\footnotetext{
${ }^{10}$ The computation tree of a Maude program $M$ for a given initial state $s$ subsumes all of the possible computations of $M$ stemming from $s$.
} 


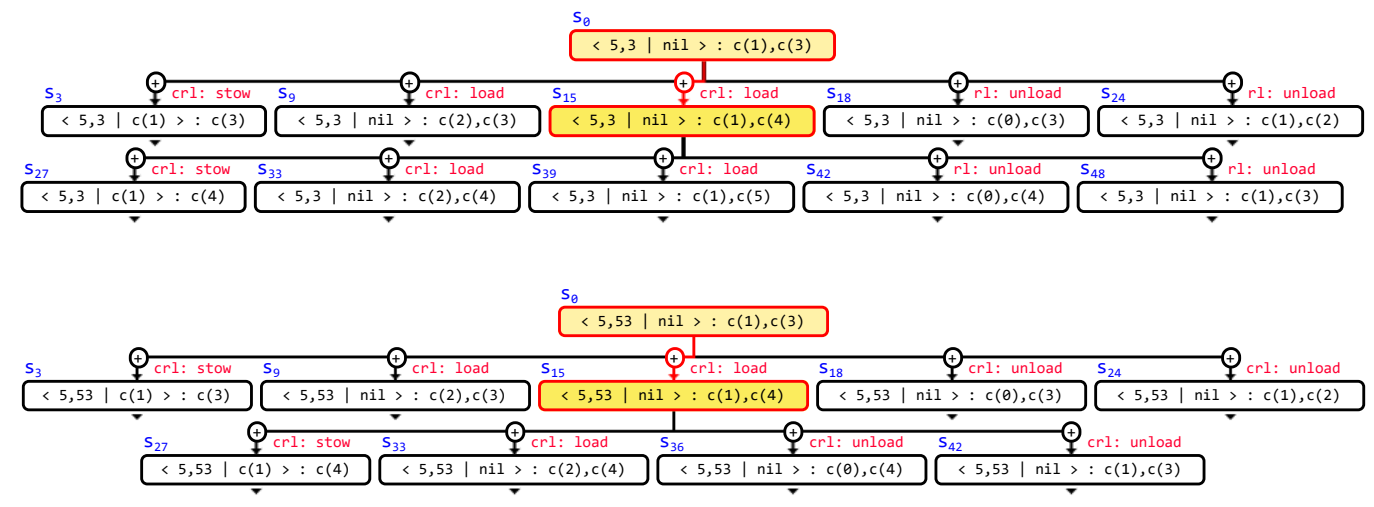

Figure 5: (Above) Fragment of the computation tree of the CONTAINER-TERMINAL module. (Below) Fragment of the computation tree of the CONTAINER-TERMINAL-FIX module.

\subsection{Experimental Results}

In order to evaluate the performance of the ÁTAME system, we endowed several Maude programs with system assertions, and we used the system to correct the programs w.r.t. the assertions. In all cases, the system assertions and program faults chosen are representative of typical deviations found in Maude programs. We benchmarked ÁTAME on the following collection of Maude programs, which are all available and fully described within the ÁTAME Web platform: Bank model, a conditional Maude specification that models a distributed banking system; Blocks World, a Maude encoding of the classical AI planning problem that consists of setting one or more vertical stacks of blocks on a table using a robotic arm; $B R P$, a Maude implementation of the Bounded Retransmission Protocol; Container, the Maude specification that models the cargo manipulation in a container terminal of the running Example 2.1; Crossing River, a Maude program that solves the well-known crossing river puzzle; Dekker, a Maude specification of Dekker's mutual exclusion algorithm; Maude NPA, an analysis tool for cryptographic protocols that takes into account the algebraic properties of cryptosystems; Philosophers, a Maude specification of the classical Dijkstra concurrency example; Semaphore, a classical mutual exclusion protocol with semaphores written in Maude; Stock Exchange, a simplified stock exchange concurrent system in which traders operate on stocks via limit orders; Webmail app, a Maude specification of a rich webmail application that provides typical email management, system administration capabilities, login/logout functionality, 
etc; Wolfram's Rule 30, a one-dimensional binary cellular automaton rule introduced by Stephen Wolfram.

\begin{tabular}{r|cc|cc|c|ccc|c|cc}
\hline \hline & \multicolumn{2}{|c|}{$\#$ Ops } & \multicolumn{2}{|c|}{$\#$ Eqs } & & & & & \\
& $\mathcal{R}$ & $\mathcal{R}_{f x}$ & $\mathcal{R}$ & $\mathcal{R}_{f i x}$ & $T T$ & $T_{\mathcal{R}}$ & $T_{\mathcal{R}}^{C h k}$ & $T_{\mathcal{R}_{f x}}$ & $S$ & $O_{C h k}$ & $O_{f i x}$ \\
\hline Bank Model & 112 & 120 & 38 & 246 & 5 & 17 & 101 & 26 & 0.74 & 4.94 & 0.53 \\
Blocks World & 89 & 104 & 12 & 194 & 3 & 19 & 37 & 31 & 0.16 & 0.95 & 0.63 \\
BRP & 23 & 26 & 12 & 27 & 2 & 5 & 23 & 7 & 0.70 & 3.6 & 0.4 \\
Container & 90 & 104 & 20 & 208 & 5 & 14 & 80 & 19 & 0.76 & 4.71 & 0.36 \\
Crossing River & 20 & 33 & 14 & 58 & 1 & 6 & 20 & 7 & 0.65 & 2.33 & 0.17 \\
Dekker & 126 & 161 & 25 & 307 & 7 & 40 & 98 & 51 & 0.48 & 1.45 & 0.28 \\
Maude NPA & 46 & 75 & 11 & 128 & 3 & 33 & 71 & 36 & 0.50 & 1.15 & 0.09 \\
Philosophers & 51 & 64 & 12 & 122 & 3 & 12 & 36 & 15 & 0.59 & 2 & 0.25 \\
Semaphore Problem & 49 & 60 & 10 & 109 & 2 & 7 & 16 & 9 & 0.44 & 1.29 & 0.29 \\
Stock Exchange & 179 & 192 & 106 & 473 & 13 & 36 & 103 & 46 & 0.55 & 1.86 & 0.28 \\
Webmail app & 317 & 409 & 191 & 1044 & 51 & 138 & 271 & 178 & 0.34 & 0.96 & 0.29 \\
Wolfram's Rule 30 & 49 & 60 & 13 & 117 & 3 & 8 & 20 & 10 & 0.5 & 1.5 & 0.25 \\
\hline \hline
\end{tabular}

Table 1: Experimental results of the correction technique.

All of the experiments were conducted on an Intel Xeon E5-1660 3.3GHz CPU with $64 \mathrm{~GB}$ RAM. Table 1 summarizes our results. We have considered five assertions per benchmark (except for the case of the Container program which includes the three assertions of Example 3.1). The \#Ops column (resp. the \#Eqs column) records the number of operator declarations (resp. the number of equations) in both the original program $\mathcal{R}$ and the statically repaired program $\mathcal{R}_{f i x}$. Column $T T$ measures the transformation time (in $\mathrm{ms}$ ) that is required to compute the program corrections for the programs in the considered benchmark set. We also measure the average execution time (in ms) of 10 computations in the original program $\mathcal{R}$ with and without assertion checking (columns $T_{\mathcal{R}}$ and $T_{\mathcal{R}}^{C h k}$, respectively) and in the repaired program $\mathcal{R}_{f i x}$ (column $T_{\mathcal{R}_{f i x}}$ ). More specifically, execution times in $T_{\mathcal{R}}^{\text {Chk }}$ have been computed in the extended Maude runtime environment of [2] that adds assertion-checking capabilities to the standard Maude rewrite engine. This way, any state transition (i.e. rewrite rule application) $t \rightarrow t^{\prime}$ is enabled in the extended runtime environment if an only if the state $t^{\prime}$ and all of its subterms meet the system assertions under examination. Note that the advantage of our correction transformation w.r.t. [2] is precisely in statically encoding the necessary checks to prevent violations into Maude programs, instead of doing unnecessary checks at runtime.

All of the executions consist of about 500 Maude computation steps, which amounts to 5,000 rewrite steps on average including equational sim- 
plification steps. In column $S$, we report the total speedups $\left(1-T_{\mathcal{R}_{\text {fx }}} / T_{\mathcal{R}}^{C h k}\right)$ that we achieve w.r.t. the highly optimized executions with runtime assertion checking of [2]. Finally, we record the overheads of the execution times for the original program $\mathcal{R}$ with respect to: 1 ) the monitored execution times for $\mathcal{R}$, i.e., the ratio $\left(T_{\mathcal{R}}^{C h k}-T_{\mathcal{R}}\right) / T_{\mathcal{R}}$ (in column $O_{C h k}$, taken from [2]); and 2) the execution times of the repaired program, i.e., the ratio $\left(T_{\mathcal{R}_{f i x}}-T_{\mathcal{R}}\right) / T_{\mathcal{R}}$ (in column $O_{f i x}$ ). These overheads indicate the relative slowdown due to runtime assertion checking (column $O_{C h k}$ ) and to the evaluation of the extra conditions inserted by the correction transformation (column $O_{f i x}$ ).

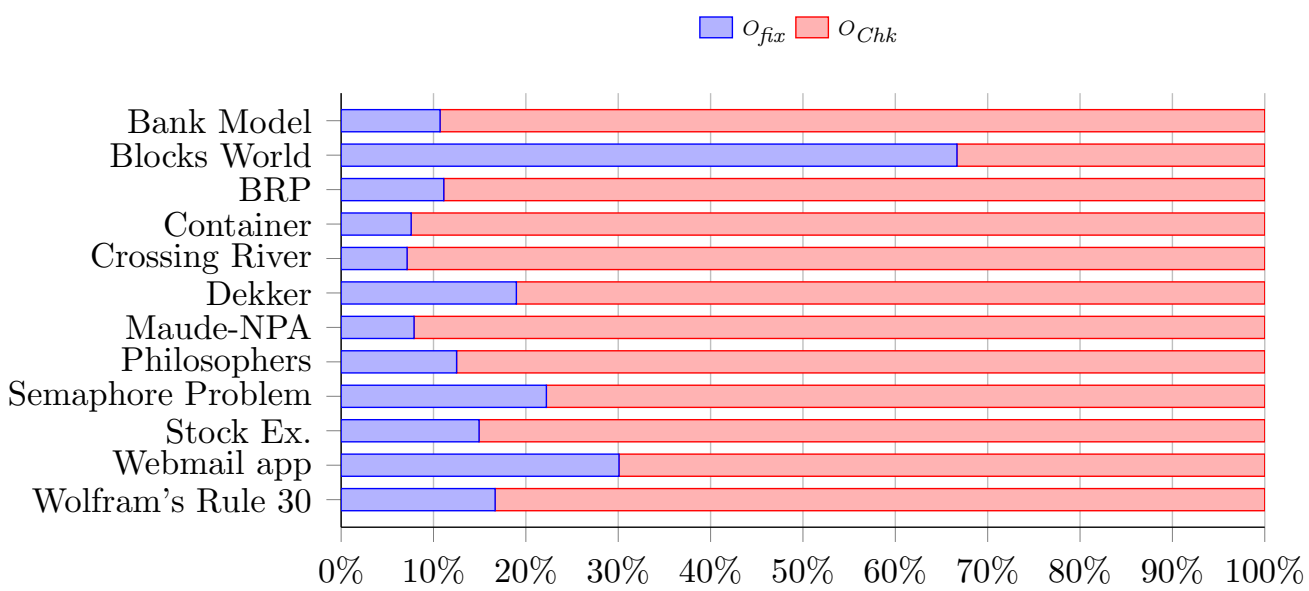

Figure 6: Overhead comparison $O_{f i x}$ vs. $O_{C h k}$.

Our figures show that, on average, the increasing in the number of equations grows linearly to the number of newly declared operators and the size of the corrected code is 2.8 times the size of the original code. To fairly calculate this ratio, we compared the actual size of the original code - which is the size of the user-defined specification plus the size of the extra definitions that are implicitly included in each imported module (e.g. INT, RAT, BOOL) - with the size of its corrected version in which extra definitions of the imported modules are explicit. In exchange for that, the correction transformation has a positive impact on the execution times w.r.t. the monitored execution times. As expected, the corrected program $\mathcal{R}_{f i x}$ is typically slower than the original program $\mathcal{R}$; nevertheless, it exhibits a better performance than $\mathcal{R}$ when run with assertion-checking enabled. Indeed, in all cases $T_{\mathcal{R}} \leq T_{\mathcal{R}_{f i x}} \leq T_{\mathcal{R}}^{C h k}$. Moreover, the average value of the speedups in column $S$ is 0.53 , which means that running the corrected program $\mathcal{R}_{f i x}$ is $53 \%$ faster than the monitored 
execution of $\mathcal{R}$.

The overheads $O_{f i x}$ and $O_{C h k}$ are graphically compared in Figure 6. The results obtained are quite satisfactory with an average value of the overheads in column $O_{f i x}$ of 0.38 , which is $14.23 \%$ of the average value (2.67) of the overheads in column $O_{C h k}$ for the same benchmark programs.

As for the time necessary for computing the program corrections, it is almost negligible (a few milliseconds) as witnessed by the data in column $T T$. Indeed, the worst case is $51 \mathrm{~ms}$ for the Webmail specification. The time for inferring the repairs is in any case a small portion of the total execution time.

If assertions are complex (e.g., they involve recursive conditional computations), as in the Bank Model, our transformation can improve the execution time significantly w.r.t. the execution with dynamic assertion checking. And even when the improvement is small or not measurable (e.g., Blocks World), the correction transformation is useful since the transformed program is demonstrably safe and corrections are generated fast enough that they could be computed during active development, thus reducing the debugging burden.

\section{Related Work and Conclusion}

Automated program correction and related problems are not new, with proposed techniques ranging from semantic analysis to stochastic search [12]. Research in this area holds promise for reducing software maintenance costs due to buggy code. A number of techniques have been developed for the code repair problem, i.e., the general problem of computing modifications to a buggy program in order to obtain a new program that satisfies a suitable specification of the expected program behavior, or user intent. Such a specification can either be expressed as sets of passing and failing test cases, functional specifications, reference implementations, program models, sets of logic properties, examples, traces, assertions, summaries or code contracts. For instance, Gopinath et al. [10] use behavioral specifications to fix buggy Java programs, and they use the SAT-based Alloy tool-set to prune any nondeterminism that could be introduced by the repair actions. Autofix [25] bridges the gap between specification-based and test-based repairs by using Eiffel contracts to correct violations of simple assertions that are formulated using Boolean methods that are already present in the program. As for the repair synthesis itself, it can be based on different search techniques, such 
as enumerative and heuristic search, deduction, constraint-solving, symbolic methods, or some combination of these [12]. Other approaches for fixing code are based on statistical fault localization, evolutionary computation, or game theory. Dynamic patch generation can also be achieved by runtime monitoring and instrumentation. For a detailed discussion, see [12, 11, 13] and references therein.

More closely related to our work is the concept of automated program repair of [14, a change to a program source that removes bad execution traces while increasing the number of good traces by applying abstract interpretation. A bad run is one that violates a given specification either provided by the programmer (e.g., as contracts), or provided by language semantics (e.g., division by zero, null pointer, etc.). A good run is one that meets all specifications of the original program. Beyond the technical differences with our work, a more basic difference is in the intention. While [14 aims to automatically produce a collection of fixes for faulty programs that are not necessarily applied in an automatic way ${ }^{11}$, we are looking to reinforce software quality by automatically generating program corrections from a set of safety assertions so that runtime checking can be safely omitted because no crashes can occur at runtime due to assertion violation. For a recent survey on automatic software repair, see [9].

In [23] a generic strategy is defined to ensure that a Maude program satisfies a set of state invariants that can be expressed in different logics. This is achieved by imposing (on top of Maude) a programmed strategy that dynamically drives the system's execution in such a way that some state transitions are avoided so that every system state complies with the constraints. In contrast, our methodology is static and enforces the assertions by transforming the program code so that the system constraints are verified by construction.

The correction methodology that we propose can be very useful for a programmer who wants to correct a program w.r.t. a preliminary version which was written with no safety concerns. Our approach can also be applied to automatically finding fixes for programs with incomplete specifications given by system assertions while keeping the transformed programs as close as possible to the original ones.

\footnotetext{
${ }^{11}$ In [14], repairs cannot be applied automatically since, in general, there is more than one repair possible for a given bug or warning.
} 
Also, it is worth noting that the core idea of our correction transformation can be applied to virtually any rewriting-based, programming language: from simple term rewriting systems to the most widespread functional languages such as Haskell and Erlang. Indeed, the proposed correction method transforms program rules into guarded program rules whose conditions supersede the system assertion checks and are simply evaluated by using the language rewriting infrastructure. Therefore, this checking mechanism can be embedded into any setting that supports rewriting with an effort that depends on the complexity of the chosen formal framework.

As future work, we plan to improve the expressiveness of our assertion language that currently supports only safety constraints (e.g. state invariants). More concretely, the idea is to extend the language with new constructs that allow one to predicate not only over single states but also over sequences of states. This way we could, i.e., specify and enforce liveness and other more convoluted, temporal properties.

\section{Acknowledgements}

We thank the referees for their careful reading of a previous version of this article and their very helpful suggestions to improve the presentation. We also thank Santiago Escobar for useful discussions concerning the optimization of Maude programs.

\section{References}

[1] M. Alpuente, D. Ballis, F. Frechina, J. Sapiña, Exploring Conditional Rewriting Logic Computations, Journal of Symbolic Computation 69 (2015) 3-39.

[2] M. Alpuente, D. Ballis, F. Frechina, J. Sapiña, Assertion-based Analysis via Slicing with ABETS, Theory and Practice of Logic Programming 16 (5-6) (2016) 515-532.

[3] M. Alpuente, D. Ballis, F. Frechina, J. Sapiña, Debugging Maude Programs via Runtime Assertion Checking and Trace Slicing, Journal of Logical and Algebraic Methods in Programming 85 (2016) 707-736.

[4] Anima, The Anima Website, Available at: http://safe-tools.dsic. upv.es/anima, 2015. 
[5] M. Clavel, F. Durán, S. Eker, S. Escobar, P. Lincoln, N. Martí-Oliet, J. Meseguer, C. Talcott, Maude Manual (Version 2.7.1), Tech. Rep., SRI International Computer Science Laboratory, available at: http: //maude.cs.uiuc.edu/maude2-manual/, 2016.

[6] M. Clavel, F. Durán, S. Eker, P. Lincoln, N. Martí-Oliet, J. Meseguer, C. Talcott, All About Maude: A High-Performance Logical Framework, vol. 4350 of Lecture Notes in Computer Science, Springer-Verlag, 2007.

[7] F. Durán, S. Eker, P. Lincoln, J. Meseguer, Principles of Mobile Maude, in: Agent Systems, Mobile Agents, and Applications, Second International Symposium on Agent Systems and Applications and Fourth International Symposium on Mobile Agents, ASA/MA 2000, Proceedings, vol. 1882 of Lecture Notes in Computer Science, Springer, 73-85, 2000.

[8] F. Durán, J. Meseguer, On the Church-Rosser and Coherence Properties of Conditional Order-sorted Rewrite Theories, The Journal of Logic and Algebraic Programming 81 (7-8) (2012) 816-850.

[9] L. Gazzola, D. Micucci, L. Mariani, Automatic software repair: a survey, in: Proceedings of the 40th International Conference on Software Engineering, ICSE 2018, Gothenburg, Sweden, May 27 - June 03, 2018, $1219,2018$.

[10] D. Gopinath, M. Z. Malik, S. Khurshid, Specification-Based Program Repair Using SAT, in: Proceedings of the 17th International Conference on Tools and Algorithms for Construction and Analysis of Systems (TACAS 2011), vol. 6605 of Lecture Notes in Computer Science, Springer, 173-188, 2011.

[11] C. Goues, S. Forrest, W. Weimer, Current Challenges in Automatic Software Repair, Software Quality Journal 21 (2013) 421-443.

[12] S. Gulwani, O. Polozov, R. Singh, Program Synthesis, Foundations and Trends in Programming Languages 4 (2017) 1-119.

[13] S. Kim, C. L. Goues, M. Pradel, A. Roychoudhury, Automated Program Repair (Dagstuhl Seminar 17022), Dagstuhl Reports 7 (2017) 19-31.

[14] F. Logozzo, T. Ball, Modular and Verified Automatic Program Repair, in: Proceedings of the 27th Annual ACM SIGPLAN Conference on 
Object-Oriented Programming, Systems, Languages, and Applications (OOPSLA 2012), Association for Computing Machinery, 133-146, 2012.

[15] S. Lucas, J. Meseguer, Operational Termination of Membership Equational Programs: the Order-Sorted Way, Electr. Notes Theor. Comput. Sci. 238 (3) (2009) 207-225.

[16] M. -O. Stehr, J. Meseguer, P. C. Ölveczky, Rewriting Logic as a Unifying Framework for Petri Nets, in: Unifying Petri Nets, Advances in Petri Nets, vol. 2128 of Lecture Notes in Computer Science, Springer, 250-303, 2001.

[17] J. Meseguer, Conditional Rewriting Logic as a Unified Model of Concurrency, Theoretical Computer Science 96 (1) (1992) 73-155.

[18] J. Meseguer, C. Talcott, Semantic Models for Distributed Object Reflection, in: Proceedings of 16th European Conference on Object-Oriented Programming (ECOOP 2002), vol. 2374 of Lecture Notes in Computer Science, Springer, 1-36, 2002.

[19] J. Meseguer, P. Thati, Symbolic Reachability Analysis Using Narrowing and its Application to Verification of Cryptographic Protocols, Electronic Notes in Theoretical Computer Science 117 (2005) 153-182.

[20] Y. Pei, C. Furia, M. Nordio, Y. Wei, B. Meyer, A. Zeller, Automated Fixing of Programs with Contracts, IEEE Transactions on Software Engineering 40 (5) (2014) 427-449.

[21] C. Rocha, J. Meseguer, C. Muñoz, Rewriting Modulo SMT and Open System Analysis, in: Proceedings of the 10th International Workshop on Rewriting Logic and its Applications (WRLA 2014), vol. 8663 of Lecture Notes in Computer Science, Springer, 247-262, 2014.

[22] C. Rocha, J. Meseguer, C. A. Muñoz, Rewriting Modulo SMT and Open System Analysis, Journal of Logical and Algebraic Methods in Programming 86 (2017) 269-297.

[23] M. Roldán, F. Durán, A. Vallecillo, Invariant-driven Specifications in Maude, Science of Computer Programming 74 (10) (2009) 812-835. 
[24] TeReSe, Term Rewriting Systems, Cambridge University Press, ISBN 978-05-2139-115-3, 2003.

[25] Y. Wei, Y. Pei, C. A. Furia, L. S. Silva, S. Buchholz, B. Meyer, A. Zeller, Automated Fixing of Programs with Contracts, in: Proceedings of 19th International Symposium on Software Testing and Analysis (ISSTA 2010), Association for Computing Machinery, 61-72, 2010. 


\section{Appendix A. Proofs of Technical Results}

\section{Proof of Proposition 4.9 (operational termination preservation).}

Let $\mathcal{E}^{\mathcal{A}}=\left(\Sigma^{\mathcal{A}}, \Delta^{\mathcal{A}} \cup B\right)$ be the $\mathcal{A}$-extension of $\mathcal{E}$. Since $\mathcal{E}=(\Sigma, \Delta \cup B)$ is operationally terminating modulo $B$, we just prove that the new equations introduced by the $\mathcal{A}$-extension of $\mathcal{E}$ cannot introduce possible sources of nontermination. We distinguish three cases, one for each category of the new equations in $\Delta^{\mathcal{A}} \backslash \Delta$. By the auxiliary notation $\sharp_{P}(t)$, we denote the total number of occurrences of symbols of the set $P$ in the term $t$.

[Ren equations]. The equations that define the renaming function Ren are listed in Cases (a), (c), and (d) of Definition 4.1. Note that for each rewrite step $t_{1} \rightarrow_{\Delta^{\mathcal{A}}, B} t_{2}$ that applies any of such equations in $\Delta^{\mathcal{A}}, \sharp_{\Sigma}\left(t_{1}\right)>\sharp_{\Sigma}\left(t_{2}\right)$ or $\sharp\{$ Ren $\}\left(t_{1}\right)>\sharp\{$ Ren $\}\left(t_{2}\right)$. In other words, $t_{2}$ contains less occurrences of $\Sigma$ operators than $t_{1}$, or less occurrences of the operator Ren $\in \Sigma^{\prime}$ than $t_{1}$. The former inequality holds when Ren equations that model the recursive cases of the renaming process are applied, while the latter is met when the equation $\operatorname{Ren}(x)=x$ is applied. This straightforwardly enforces a well-founded (reduction) order that suffices to guarantee the operational termination of the renaming process. Indeed, the evaluation instances of the conditions in any Ren equation are terminating. Simply observe that such instances are always of the form $\left(x_{1} \sigma=/=i d \wedge x_{2} \sigma=/=i d\right)$, with $i d$ the identity of an operator in $\Sigma$, and $x_{1} \sigma, x_{2} \sigma \in \mathcal{T}(\Sigma, \mathcal{V} a r)$, which implies that $x_{1} \sigma, x_{2} \sigma$ are simplified in the (operationally) terminating theory $\mathcal{E}$, hence the evaluation of $\left(x_{1} \sigma=/=i d \wedge x_{2} \sigma=/=i d\right)$ terminates.

[Ren ${ }^{-1}$ equations]. This case is analogous to the previous one, since the definition of $\operatorname{Ren}^{-1}$ is analogous to the one of Ren.

[Assertion-checking equations]. Given an assertion-checking equation $e_{a}=\left(\Pi^{\prime}=\right.$ fail if $\left.\operatorname{not}\left(\operatorname{Ren}^{-1}\left(\varphi^{\prime}\right)\right)\right)$ in $\Delta^{\mathcal{A}}$, each rewrite step $t_{1}^{\prime} \rightarrow_{\Delta^{\mathcal{A}}, B} t_{2}^{\prime}$ that applies $e_{a}$ replaces a subterm of $t_{1}^{\prime}$ with an occurrence of the irreducible term fail. Actually, $\sharp_{\Sigma^{\prime}}\left(t_{1}^{\prime}\right)>\sharp_{\Sigma^{\prime}}\left(t_{2}^{\prime}\right)$. Also note that the evaluation of the corresponding instance of the associated condition $\operatorname{not}\left(\operatorname{Ren}^{-1}\left(\varphi^{\prime}\right)\right)$ is always terminating. In fact, the outermost strategy of $\operatorname{Ren}^{-1}$ guarantees that the renamed functions in such an instance are first transformed into their original versions and then evaluated by using the operationally terminating (modulo $B$ ) equational theory $\mathcal{E}$.

[Fail-detecting equations]. Similar to the previous case. Every rewrite step $t_{1}^{\prime} \rightarrow_{\Delta^{\mathcal{A}}, B} t_{2}^{\prime}$ that involves a fail-detecting equation in $\Delta^{\mathcal{A}}$ replaces a subterm of $t_{1}^{\prime}$ with an occurrence of the irreducible term fail, and $\sharp_{\Sigma^{\prime}}\left(t_{1}^{\prime}\right)>$ 
$\sharp_{\Sigma^{\prime}}\left(t_{2}^{\prime}\right)$. Also, conditions of the form $x=/=$ fail in the conditional part of a fail-detecting equation rule cannot introduce nontermination since $x$ is always bound to renamed terms that are irreducible in $\mathcal{E}^{\mathcal{A}}$.

In order to prove Proposition 4.10, we recall the notions of sort-decreasing, confluent, and Church-Rosser equational theory.

\section{Definition Appendix A.1 (sort-decreasing equational theory)}

The equational theory $\mathcal{E}=(\Sigma, \Delta, B)$ is sort-decreasing iff all of the equations $(u=v$ if $C)$ in $\Delta$ are sort decreasing, i.e., for every substitution $\sigma$, if $C \sigma$ evaluates to true in $\mathcal{E}$, then $l s(u \sigma) \geq l s(v \sigma)$.

\section{Definition Appendix A.2 (confluent equational theory)}

The equational theory $\mathcal{E}=(\Sigma, \Delta, B)$ is confluent (modulo B) iff $\rightarrow_{\Delta, B}$ is confluent modulo $B^{12}$, i.e., for any $t \rightarrow_{\Delta, B}^{*} u$ and $t \rightarrow_{\Delta, B}^{*} v$, there exist terms $w_{1}$ and $w_{2}$ such that $u \rightarrow_{\Delta, B}^{*} w_{1}, v \rightarrow_{\Delta, B}^{*} w_{2}$, and $w_{1}={ }_{B} w_{2}$.

\section{Definition Appendix A.3 (Church-Rosser equational theory)}

The equational theory $\mathcal{E}=(\Sigma, \Delta, B)$ is Church-Rosser (modulo $B$ ) iff $\mathcal{E}$ is sort-decreasing and confluent modulo $B$.

In practice, checking the confluence of a conditional order-sorted, operationally terminating ${ }^{13}$ equational theory $\mathcal{E}=(\Sigma, \Delta \cup B, R)$ boils down to the joinability of the conditional critical pairs of $\mathcal{E}$, as shown in [8].

Definition Appendix A.4 (conditional critical pair) Let $\mathcal{E}=(\Sigma, \Delta$, $B)$ be an equational theory. Let $\left(l_{1}=r_{1}\right.$ if $\left.C_{1}\right)$ and $\left(l_{2}=r_{2}\right.$ if $\left.C_{2}\right)$ be two (renamed apart) equations in $\Delta$ such that $l_{1 \mid p} \sigma={ }_{B} l_{2} \sigma$, for some nonvariable position $p \in \mathcal{P} \operatorname{Pos}\left(l_{1}\right)$ and substitution $\sigma$. Then, the triple $\left(C_{1} \sigma \wedge C_{2} \sigma \Rightarrow\right.$ $\left.\left(l_{1}\left[r_{2}\right]_{p}\right) \sigma=r_{1} \sigma\right)$ is a (conditional) critical pair in $\mathcal{E}$.

\footnotetext{
${ }^{12}$ Actually, confluence should be defined w.r.t. the relation $\rightarrow_{\Delta / B}$. However, under the (strict) $B$-coherence assumption, relations $\rightarrow_{\Delta, B}$ and $\rightarrow_{\Delta / B}$ are equivalent. Additionally, for any $B$ that includes combinations of associativity and/or commutativity and/or unity axioms, it is possible to make any specification $B$-coherent by using a terminating completion procedure ([6], Section 4.8).

${ }^{13}$ In [8, the related notion of quasi-decreasingness is assumed that is equivalent to operational termination, which is the property checked by Maude's MTT tool to prove the termination of an order-sorted conditional rewrite theory.
} 


\section{Definition Appendix A.5 (trivially joinable critical pair)}

Let $\mathcal{E}=(\Sigma, \Delta, B)$ be an equational theory. Let $(C \Rightarrow u=v)$ be a (conditional) critical pair in $\mathcal{E}$. We say that $(C \Rightarrow u=v)$ is trivially joinable iff there exist terms $w_{1}$ and $w_{2}$ such that $u \rightarrow_{\Delta, B}^{*} w_{1}, v \rightarrow_{\Delta, B}^{*} w_{2}$, and $w_{1}=_{B} w_{2}$.

Proof of Proposition 4.10 (Church-Rosser preservation). Let $\mathcal{E}^{\mathcal{A}}=$ $\left(\Sigma^{\mathcal{A}}, \Delta^{\mathcal{A}} \cup B\right)$ be the $\mathcal{A}$-extension of the equational theory $\mathcal{E}=(\Sigma, \Delta \cup B)$. By Definition 4.7, $\Sigma^{\mathcal{A}}=\Sigma \cup \Sigma^{\prime} \cup\{$ fail : $\rightarrow \top\}$ and $\Delta^{\mathcal{A}}=\Delta \cup \Delta^{\prime} \cup \mathrm{A} \cup \mathrm{F}$. To prove the Church-Rosser property for $\mathcal{E}^{\mathcal{A}}=\left(\Sigma^{\mathcal{A}}, \Delta^{\mathcal{A}} \cup B\right)$, we need to show that (i) $\mathcal{E}^{\mathcal{A}}$ is sort-decreasing, and (ii) $\mathcal{E}^{\mathcal{A}}$ is confluent modulo $B$.

(i) By hypothesis, $\mathcal{E}=(\Sigma, \Delta \cup B$ ) is Church-Rosser (modulo $B$ ), thus $\mathcal{E}=$ $(\Sigma, \Delta \cup B)$ is sort-decreasing. Hence, to prove the sort-decreasingness of $\mathcal{E}^{\mathcal{A}}$, we just need to prove that all of the equations in $\left(\Delta^{\mathcal{A}} \backslash \Delta\right)=$ $\left(\Delta^{\prime} \cup \mathrm{A} \cup \mathrm{F}\right)$ are sort-decreasing. In other words, we have to prove that for every substitution $\sigma$ and equation $(u=v$ if $C) \in\left(\Delta^{\prime} \cup \mathrm{A} \cup \mathrm{F}\right)$, $l s(u) \geq l s(v)$, if $C \sigma$ evaluates to true in $\mathcal{E}^{\mathcal{A}}$. We consider separately the cases when $(u=v$ if $C)$ is in $\Delta^{\prime}$, in $\mathrm{A}$, and in F. First note that, by assumption, the signature $\Sigma$ is preregular, and that the new operators in $\Sigma^{\prime} \cup\{$ fail: $\rightarrow \top\}$ trivially do not break preregularity. This implies that every term $t$ built upon $\Sigma^{\mathcal{A}}=\Sigma \cup \Sigma^{\prime} \cup\{$ fail : $\rightarrow \top\}$ has a least sort $l s(t)$.

$(u=v$ if $C) \in \Delta^{\prime}$. Equations in $\Delta^{\prime}$ define the polymorphic operators Ren : $\top \rightarrow \top$ and $\operatorname{Ren}^{-1}: \top \rightarrow \top$. We only show sort-decrasingess of Ren equations since the argument to prove sort-decrasingess of $\operatorname{Ren}^{-1}$ is perfectly analogous. Since the distinguished sort $T$ is actually a sort placeholder for any known sorts that is consistently instantiated during term simplification, and every operator $\left(f: s_{1} \ldots s_{n} \rightarrow s\right) \in$ $\Sigma$ has a single renamed counterpart $\left(f^{\prime}: s_{1} \ldots s_{n} \rightarrow s\right) \in \Sigma$ that has the same sort declaration of $f$, we have that, for each equation $(\operatorname{Ren}(u)=v$ if $C) \in \Delta^{\prime}$, and for each substitution $\sigma$ such that $C \sigma$ evaluates to true in $\Sigma_{\mathcal{A}}, \operatorname{Ren}(u) \sigma$ and $v \sigma$ have the same sort. Hence $l s(\operatorname{Ren}(u) \sigma) \geq l s(v \sigma)$.

$(u=v$ if $C) \in \mathrm{A}$. Every equation in $\mathcal{A}$ has the form $\Pi^{\prime}=$ fail if $C$. Since fail is a polymorphic constant, i.e., fail $: \rightarrow T$, we have that, for each substitution $\sigma$ such that $C \sigma$ evaluates to true in $\Sigma_{\mathcal{A}}, \Pi^{\prime} \sigma$ and fail have the same sort. Thus, $l s\left(\Pi^{\prime} \sigma\right) \geq l s($ fail $)=l s($ fail $\sigma)$. 
$(u=v$ if $C) \in \mathrm{F}$. Every equation in $\mathcal{A}$ has the form

$$
f^{\prime}\left(x_{1}, \ldots x_{i-1} \text {, fail, } x_{i+1} \ldots x_{n}\right)=\text { fail if } C
$$

where $i=0, \ldots, n$, and $f^{\prime} \in \Sigma^{\prime}$. Since fail is a polymorphic constant, i.e., fail $: \rightarrow \top$, we have that, for each substitution $\sigma$ such that $C \sigma$ evaluates to true in $\Sigma_{\mathcal{A}}, f^{\prime}\left(x_{1}, \ldots x_{i-1}\right.$, fail, $\left.x_{i+1} \ldots x_{n}\right) \sigma$ and fail have the same sort. Thus, $l s\left(f^{\prime}\left(x_{1}, \ldots x_{i-1}\right.\right.$, fail, $\left.\left.x_{i+1} \ldots x_{n}\right) \sigma\right) \geq l s($ fail $)=$ $l s($ fail $\sigma)$.

(ii) By Proposition $4.9, \mathcal{E}^{\mathcal{A}}$ is operationally terminating modulo $B$ since $\mathcal{E}$ is operationally terminating modulo $B$. Hence, it suffices to prove that the sufficient condition in [8] for confluence modulo $B$ of a conditional order sorted, operationally terminating equational theory holds for $\mathcal{E}^{\mathcal{A}}$. We prove it by showing that the new equations in $\Delta^{\prime} \cup A \cup F$ only introduce trivially joinable critical pairs.

We list below the critical pairs that can be introduced by the new equations of $\mathcal{E}^{\mathcal{A}}$, and we reason by cases to prove their trivial joinability. Note that the operators Ren and $\operatorname{Ren}^{-1}$ in $\Delta^{\prime}$ do not produce factual critical pairs due to their evaluation strategies. Indeed, an innermost evaluation strategy is enforced to terms of the form $\operatorname{Ren}(t)$ that applies the Ren operator only to a canonical form $t$. Thus, the evaluation of $t$ does not interfere with the renaming process. Dually, $\operatorname{Ren}^{-1}$ uses an outermost evaluation strategy on terms of the form $\operatorname{Ren}^{-1}\left(t^{\prime}\right)$ which forbids any rewriting inside $t^{\prime}$.

Critical pairs between equations of $\mathrm{F}$. Note that all the critical pairs between two fail-detecting equations in $F$ arise at the root position of the equation left-hand-sides and thus have the following form $C \Rightarrow$ fail $=$ fail with $C$ a possibly empty condition. These critical pairs are trivially joinable.

Critical pairs between equations of $\mathrm{A}$ and $\mathrm{F}$. Consider an assertionchecking equation of $e_{a}=\left(\Pi^{\prime}=\right.$ fail if $\left.\operatorname{not}\left(\operatorname{Ren}^{-1}\left(\varphi^{\prime}\right)\right)\right)$ in $\mathcal{A}$ and a fail-detecting equation $f \in \mathrm{F}$. Then, the only possible form of a critical pair between $e_{a}$ and $f$ is $\left(C \Rightarrow \Pi^{\prime}[\text { fail }]_{p}=\right.$ fail $)$, for some $p \in \mathcal{P}$ os $\left(\Pi^{\prime}\right)$. Again, these pairs are all trivially joinable, since $\Pi^{\prime}[\text { fail }]_{p} \rightarrow_{\mathrm{F}, B}^{*}$ fail. 
Proof of Proposition 4.11 (completeness of the $\mathcal{A}$-extension). Let $\mathcal{E}=(\Sigma, \Delta \cup B)$ be an equational theory. Let $a \in \mathcal{A}$ be a system assertion of the form $\Pi \mid \varphi$ and $s t \in \mathcal{T}(\Sigma, \mathcal{V})$ be a system state such that $(s t) \downarrow_{\Delta, B} \not=\Pi \mid \varphi$. Hence, there exist a position $w \in \mathcal{P}$ os $(s t)$ and a substitution $\sigma$ such that $\left((s t) \downarrow_{\Delta, B}\right)_{\mid w}={ }_{B} \Pi \sigma$ and $\varphi \sigma \rightarrow_{\Delta, B}^{*}$ false. By Definition 4.7, there exists the assertion checking equation

$$
e_{\Pi \mid \varphi}=\left(\Pi^{\prime}=\text { fail if } \operatorname{not}\left(\operatorname{Ren}^{-1}\left(\varphi^{\prime}\right)\right)\right) \in \Delta^{\mathcal{A}}
$$

Now, it is immediate to see that

$$
(\operatorname{Ren}(s t))_{\mid w} \stackrel{e_{\Pi \mid \varphi}, \sigma^{\prime}, w}{\rightarrow}, \mathcal{A} \cup B \text { fail }
$$

with the substitution $\sigma^{\prime}=\left\{x / t^{\prime} \mid x / t \in \sigma\right\}$. Indeed,

- $\operatorname{Ren}(s t)_{\mid w}={ }_{B}\left(\operatorname{Ren}(s t)_{\mid w}\right) \downarrow_{\Delta, B}={ }_{B} \Pi^{\prime} \sigma^{\prime}$, since $s t_{\mid w}={ }_{B}\left(s t_{\mid w}\right) \downarrow_{\Delta, B}={ }_{B}$ $\Pi \sigma$, and $\operatorname{Ren}\left(s t_{\mid w}\right)$ and $\Pi^{\prime}$ are respectively the corresponding renamed versions of the canonical form $\left(s t_{\mid w}\right) \downarrow_{\Delta, B}$ of $s t_{\mid w}$ and $\Pi$ in $\left(\Sigma^{\mathcal{A}}, \Delta^{\mathcal{A}} \cup B\right)$;

- $\operatorname{not}\left(\operatorname{Ren}^{-1}\left(\varphi^{\prime}\right)\right) \sigma^{\prime}=\operatorname{not}\left(\operatorname{Ren}^{-1}\left(\varphi^{\prime} \sigma^{\prime}\right)\right)=\operatorname{not}(\varphi \sigma) \rightarrow_{\Delta{ }^{\mathcal{A}} \cup B}^{*}$ true because $\varphi \sigma \rightarrow_{\Delta, B}^{*}$ false and $\sigma^{\prime}$ only introduces in $\varphi$ renamed bindings of $\sigma$ (in fact, $\left.\sigma^{\prime}=\left\{x / t^{\prime} \mid x / t \in \sigma\right\}\right)$.

Now, let us prove that if $(s t) \downarrow_{\Delta, B} \not=\Pi \mid \varphi$, then $\operatorname{Ren}(s t) \rightarrow_{\Delta_{\mathcal{A}}, B}^{*}$ fail. The proof is done by induction on the position $w \in \mathcal{P}$ os $(\operatorname{Ren}(s t))$ used in step (A.1) above, i.e., $\operatorname{Ren}(s t)_{\mid w} \stackrel{e_{\Pi \mid \varphi}, \sigma^{\prime}, w}{\rightarrow}, \mathcal{A} \cup B$ fail.

$(w=\Lambda)$ Immediate, since $\operatorname{Ren}(s t)=\operatorname{Ren}(s t)_{\mid \Lambda}$ and $\operatorname{Ren}(s t)_{\mid \Lambda} \stackrel{e_{\Pi \mid \varphi}, \sigma^{\prime}, w}{\rightarrow}, \mathcal{A}_{\cup B}$ fail by Equation A.1.

$(w=i . p)$ In this case, $w=i . p \in \mathcal{P}$ os $(\operatorname{Ren}(s t))$ for some natural number $i$ that ranges from 1 to the arity of $\operatorname{root}(\operatorname{Ren}(s t))$ and some position $p$ denoting the rest of the accessing path to $\operatorname{Ren}(s t)_{\mid w}$. This implies that $\operatorname{Ren}(s t)$ is a renamed version of the canonical form $\left(s t_{\mid w}\right) \downarrow_{\Delta, B}$ of $s t$ of the form $f^{\prime}\left(t_{1}^{\prime}, \ldots, t_{n}^{\prime}\right)$ with $\operatorname{Ren}(s t)_{\mid w}=t_{i \mid p}^{\prime}\left(\right.$ and, hence $\left.\left(s t_{\mid w}\right) \downarrow_{\Delta, B}=t_{i \mid p}\right)$. Because $\left(s t_{\mid w}\right) \downarrow_{\Delta, B}=t_{i \mid p}$ and $s t \not \models \Pi \mid \varphi$, we also have that $t_{i} \not \models \Pi \mid \varphi$. By applying the induction hypothesis, we get $t_{i}^{\prime} \rightarrow_{{ }_{\Delta}^{\mathcal{A}} \cup B}^{*}$ fail.

Furthermore, by Definition 4.7, there exists the equation

$$
f^{\prime}\left(x_{1}, \ldots, x_{i-1} \text {, fail }, x_{i+1}, \ldots, x_{n}\right)=\text { fail } \in \Delta^{\mathcal{A}}
$$

Therefore, we can finally build the following rewrite sequence 


$$
\begin{aligned}
\operatorname{Ren}(s t)= & f^{\prime}\left(t_{1}^{\prime}, \ldots, t_{i-1}^{\prime}, t_{i}^{\prime}, t_{i+1}^{\prime}, \ldots, t_{n}^{\prime}\right) \rightarrow_{\Delta \mathcal{A} \cup B}^{*} \\
& f^{\prime}\left(t_{1}^{\prime}, \ldots, t_{i-1}^{\prime}, \text { fail }, t_{i+1}^{\prime}, \ldots, t_{n}^{\prime}\right) \rightarrow^{*}{ }_{\Delta \mathcal{A} \cup B}
\end{aligned}
$$

fail.

Let us proceed with the proof of coherence preservation. In [8, Theorem 5], it is proved that checking the coherence of a conditional order-sorted, operationally terminating rewrite theory $(\Sigma, \Delta \cup B, R)$ can be reduced to checking the joinability of conditional critical pairs between equations in $\Delta$ and rules in $R$ (plus additional conditions required in non-overlap cases). By exploiting this result, we can prove the following proposition.

Proof of Proposition 5.5 (coherence preservation). Let $\mathcal{R}=(\Sigma, \Delta \cup$ $B, R)$ be a topmost rewrite theory that meets the conditions of $[8$, Theorem 5], and $\mathcal{A}$ be an assertion set. Then, by [8, Theorem 5], $\mathcal{R}$ is coherent. In order to prove that the $\mathcal{A}$-extension of $\mathcal{R}, \mathcal{R}^{\prime}=\left(\Sigma^{\mathcal{A}}, \Delta^{\mathcal{A}} \cup B, R^{\mathcal{A}}\right)$, is coherent, we show that the sufficient conditions of [8, Theorem 5] are also met by $\mathcal{R}^{\prime}$, thus $\mathcal{R}^{\prime}$ is coherent.

Let us analyze the required conditions in [8] which have been reformulated to our setting.

Condition $1\left(\Sigma^{\mathcal{A}}, \Delta^{\mathcal{A}} \cup B\right)$ models an admissible module.

This condition is met since $\mathcal{R}=(\Sigma, \Delta \cup B, R)$ specifies an admissible module, hence all the equations in $\Delta$ are admissible. Furthermore, the new equations in $\Delta^{\mathcal{A}} \backslash \Delta$ are also all admissible.

Condition $2\left(\Sigma^{\mathcal{A}}, \Delta^{\mathcal{A}} \cup B\right)$ is Church-Rosser modulo B.

$\mathcal{R}=(\Sigma, \Delta \cup B, R)$ is Church-Rosser and operationally terminating modulo $B$, hence $\left(\Sigma^{\mathcal{A}}, \Delta^{\mathcal{A}} \cup B\right)$ is Church-Rosser modulo $B$ by Proposition 4.10 .

Condition 3 All of the rewrite rules in $R^{\mathcal{A}}$ do not contain rewrite expressions and are $B$-coherent.

By Definition 5.4, the rewrite rules in $\mathcal{R}^{\mathcal{A}}$ have the form $\lambda \Rightarrow \rho$ if $C \wedge$ $\operatorname{Ren}(\rho)=/=$ fail, and $(\lambda \Rightarrow \rho$ if $C) \in R$. Now, it suffices to note that, by assumption, the rewrite rules in $R$ do not contain rewrite expressions, 
hence $(C \wedge \operatorname{Ren}(\rho)=/=$ fail $)$ does not contain any rewrite expression, as well.

Finally note that $B$-coherence of rules in $R^{\mathcal{A}}$ is not a concern, since a completion method can be applied to $R^{\mathcal{A}}$ to automatically achieve $B$-coherence [6, Section 4.8] for any $B$ that includes combinations of associativity and/or commutativity and/or unity axioms.

Condition $4 \mathcal{R}^{\prime}$ is operationally terminating.

By Proposition 4.9, $\mathcal{R}^{\prime}$ is operationally terminating since $\mathcal{R}$ is.

Condition 5 All critical pairs between rules in $R^{\mathcal{A}}$ and $\Delta^{A}$ are joinable.

This condition is trivially satisfied, since the renaming mechanism, encoded in the correction transformation, ensures that the new equations added to the fixed program $\mathcal{R}^{\prime}$ cannot generate critical pairs - indeed, the left-hand sides of the rules in $R^{\mathcal{A}}$ are built using the original symbols of $\mathcal{R}$, while the left-hand sides of the new, added equations are built using the primed version of the original symbols or the symbols Ren and $\operatorname{Ren}^{-1}$ that do not appear in $\Sigma$, hence no overlap is possible.

Condition 6 For every equation $(l=r$ if $C) \in \Delta^{\mathcal{A}}$, and $x \in \mathcal{V}$ ar $(l)$ such that $x$ is nonfrozen in $l$, the sort $s$ of $x$ is such that no rewriting is possible with $\rightarrow_{R^{\mathcal{A}}, B}$ for terms of such sort $s$.

By Definition 4.7, $\Sigma^{\mathcal{A}}=\Sigma \cup \Sigma^{\prime} \cup\{$ fail : $\rightarrow \top\}$ and $\Delta^{\mathcal{A}}=\Delta \cup \Delta^{\prime} \cup \mathrm{A} \cup \mathrm{F}$. Since $\mathcal{R}=(\Sigma, \Delta \cup B, R)$ is topmost, Condition 6 is trivially satisfied by all of the equations in $\Delta$. Also, note that the left-hand sides of the equations in $F$ and $A$ are built using renamed operators of the form $\left(f^{\prime}: s_{1} \ldots s_{n} \rightarrow s\right) \in \Sigma^{\prime}$, which have the same sort declaration of their original versions $\left(f: s_{1} \ldots s_{n} \rightarrow s\right) \in \Sigma$. This implies that also the equations in $\mathrm{F}$ and $\mathrm{A}$ satisfy Condition 6 (otherwise, we could derive that $\mathcal{R}$ is not topmost that is a contradiction). Finally, note that $\Delta^{\prime}$ equationally specifies the renaming operators Ren and $\operatorname{Ren}^{-1}$, whose input argument is assumed to be frozen (see Section 4). Hence, Condition 6 vacuously holds for equations in $\Delta^{\prime}$. Therefore, all of the equations in $\Delta^{\mathcal{A}}=\Delta \cup \Delta^{\prime} \cup \mathrm{A} \cup \mathrm{F}$ satisfy Condition 6 .

Since all the sufficient conditions of [8, Theorem 5] are met for $\mathcal{R}^{\mathcal{A}}$, we can conclude that $\mathcal{R}^{\mathcal{A}}$ is coherent. 
Proof of Theorem 5.6 (sound topmost correction). Let $\mathcal{R}=(\Sigma, \Delta \cup$ $B, R)$ be a topmost rewrite theory, $\mathcal{A}$ be an assertion set, and $\mathcal{R}^{\prime}=\left(\Sigma^{\mathcal{A}}, \Delta^{\mathcal{A}} \cup\right.$ $B, R^{\mathcal{A}}$ ) be the $\mathcal{A}$-extension of $\mathcal{R}$. By Definition 5.3, in order to show that $\mathcal{R}^{\prime} \leq^{\mathcal{A}} \mathcal{R}$ (i.e., $\mathcal{R}^{\prime}$ is a correction of $\mathcal{R}$ w.r.t. $\mathcal{A}$ ), we have to prove that

$i$. for all $t_{0} \rightarrow_{\mathcal{R}^{\prime}} \ldots \rightarrow_{\mathcal{R}^{\prime}} t_{n}$ s.t. $t_{0}=\mathcal{A}$, there is $t_{0} \rightarrow_{\mathcal{R}} \ldots \rightarrow_{\mathcal{R}}$ $t_{n}$, s.t. $t_{i}=\mathcal{A}, i=0, \ldots, n$

ii. for all $t_{0} \rightarrow_{\mathcal{R}} \ldots \rightarrow_{\mathcal{R}} t_{n}$, s.t. $t_{i} \models \mathcal{A}, i=0, \ldots, n$, there is $t_{0} \rightarrow_{\mathcal{R}^{\prime}}$ $\ldots \rightarrow \rightarrow_{\mathcal{R}^{\prime}} t_{n}$.

Let us first prove Claim $i$.

[Claim $i]$ Let us consider an arbitrary computation $\mathcal{C}^{\mathcal{A}}=\left(t_{0} \rightarrow_{\mathcal{R}^{\prime}} \ldots \rightarrow_{\mathcal{R}^{\prime}}\right.$ $\left.t_{n}\right)$ in $\mathcal{R}^{\prime}=\left(\Sigma^{\mathcal{A}}, \Delta^{\mathcal{A}} \cup B, R^{\mathcal{A}}\right)$ such that $t_{0} \models \mathcal{A}$. We proceed by induction on the length $n$ of the computation $\mathcal{C}^{\mathcal{A}}$.

$n=0$. Immediate, since $\mathcal{C}^{\mathcal{A}}$ does not contain any rewrite step and $t_{0} \models \mathcal{A}$.

$n>0$. The computation $\mathcal{C}^{\mathcal{A}}$ can be decomposed as follows:

$$
\mathcal{C}^{\mathcal{A}}=\left(t_{0} \rightarrow_{\mathcal{R}^{\prime}} \ldots \rightarrow_{\mathcal{R}^{\prime}} t_{n-1} \rightarrow_{\mathcal{R}^{\prime}} t_{n}\right) .
$$

Now, by applying the induction hypothesis to $t_{0} \rightarrow_{\mathcal{R}^{\prime}} \ldots \rightarrow_{\mathcal{R}^{\prime}} t_{n-1}$, we know that there exists

$$
\mathcal{C}_{n-1}^{\mathcal{A}}=\left(t_{0} \rightarrow_{\mathcal{R}} \ldots \rightarrow_{\mathcal{R}} t_{n-1}\right), \text { s.t. } t_{i}=\mathcal{A}, i=0, \ldots,(n-1)
$$

Therefore, to prove Claim $i$, we just need to show that

(a) $t_{n-1} \rightarrow_{\mathcal{R}} t_{n}$

(b) $t_{n}=\mathcal{A}$.

The proof of (a) is as follows: since $\mathcal{R}$ is topmost, we can expand $t_{n-1} \rightarrow_{\mathcal{R}^{\prime}} t_{n}$ as follows

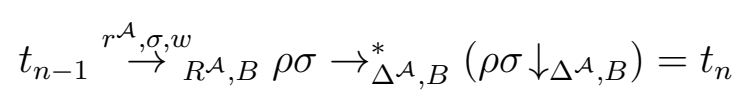

where $r^{\mathcal{A}}=(\lambda \Rightarrow \rho$ if $C \wedge \operatorname{Ren}(\rho)=/=$ fail $) \in R^{\mathcal{A}}$ is just a more restrictive variant of $r=(\lambda \Rightarrow \rho$ if $C) \in R$, thus we also have $t_{n-1} \stackrel{r, \sigma, w}{\rightarrow} R, B \rho \sigma$. Furthermore, $\rho \sigma$ does not contain any renamed operator. This implies that all the equations of $\Delta^{\mathcal{A}}$, which are used to simplify $\rho \sigma$ into 
its canonical form $\left(\rho \sigma \downarrow_{\Delta \mathcal{A}, B}\right)$, are also included in $\Delta$; hence $\rho \sigma \rightarrow_{\Delta, B}^{*}$ $\left(\rho \sigma \downarrow_{\Delta, B}\right)$ as well. Therefore,

$$
t_{n-1} \stackrel{r, \sigma, w}{\rightarrow}_{R, B} \rho \sigma \rightarrow_{\Delta, B}^{*}\left(\rho \sigma \downarrow_{\Delta, B}\right)=t_{n}
$$

To prove (b), observe that in order to enable the rewrite step

$$
t_{n-1} \stackrel{r^{\mathcal{A}}, \sigma, w}{\rightarrow} R^{\mathcal{A}, B} \rho \sigma
$$

included in $t_{n-1} \rightarrow_{\mathcal{R}^{\prime}} t_{n}$, the instantiated condition $\operatorname{Ren}(\rho \sigma)=/=$ fail of $r^{\mathcal{A}}$ must hold. Thus,

$$
\operatorname{Ren}(\rho \sigma) \nrightarrow_{\Delta^{\mathcal{A}}, B}^{*} \text { fail }
$$

which implies that $(\rho \sigma) \downarrow_{\Delta, B}=\mathcal{A}$ by Proposition 4.11 .

[Claim ii] We consider an arbitrary computation $\mathcal{C}=\left(t_{0} \rightarrow_{\mathcal{R}} \ldots \rightarrow_{\mathcal{R}}\right.$ $\left.t_{n}\right)$ s.t. $t_{i} \models \mathcal{A}, i=0, \ldots, n$. Similarly to the proof of Claim $i$, we just proceed by induction on the length $n$ of the computation $\mathcal{C}$ to show that there exists $t_{0} \rightarrow_{\mathcal{R}^{\prime}} \ldots \rightarrow_{\mathcal{R}^{\prime}} t_{n}$. Again, the base case is trivial, while the inductive case is similar to the inductive case of [Claim $i$ ] by making use of Proposition 4.11.

\section{Proof of Proposition 6.5.}

\section{(correctness and completeness of the topmost extension)}

The case when $A x=\mathrm{ACU}$ has been stated in Lemma 5.3 of [19]. Here, we prove the case when $A x=\mathrm{AC}$, which involves two extension rewrite rules, namely, $(\{X \otimes \lambda\} \Rightarrow\{X \otimes \rho\}$ if $C)$ and $(\{\lambda\} \Rightarrow\{\rho\}$ if $C)$. The remaining cases are straightforward adaptations of the following proof scheme.

We have to prove the following two implications for the case when $A x=\mathrm{AC}$ :

$(\rightarrow)$ for any term $t_{i}$ and $t_{f}$ of sort Config, $t_{i} \rightarrow_{\mathcal{R}}^{*} t_{f}$ implies $\left\{t_{i}\right\} \rightarrow_{\hat{\mathcal{R}}}^{*}\left\{t_{f}\right\}$

$(\leftarrow)$ for any term $t_{i}$ and $t_{f}$ of sort Config, $\left\{t_{i}\right\} \rightarrow_{\hat{\mathcal{R}}}^{*}\left\{t_{f}\right\}$ implies $t_{i} \rightarrow_{\mathcal{R}}^{*} t_{f}$.

$(\rightarrow)$ Assume that $t_{i} \rightarrow_{\mathcal{R}}^{*} t_{f}$, where $t_{i}$ and $t_{f}$ are arbitrary terms of sort Config. Then, $t_{i} \rightarrow_{\mathcal{R}}^{*} t_{f}$ has the form

$$
t_{i}=t_{0} \rightarrow_{\mathcal{R}} \ldots \rightarrow_{\mathcal{R}} t_{n-1} \rightarrow_{\mathcal{R}} t_{n}=t_{f} \text {, for some natural number } n \geq 0 .
$$

We proceed by induction on the length $n$ of the rewriting sequence $t_{i} \rightarrow_{\mathcal{R}}^{*} t_{f}$. 
$n=0$. Immediate, since there are no rewrite steps.

$n>0$. By induction hypothesis, we have

$$
t_{i}=t_{0} \rightarrow_{\mathcal{R}}^{*} t_{n-1} \text { implies }\left\{t_{i}\right\}=\left\{t_{0}\right\} \rightarrow_{\mathcal{R}}^{*}\left\{t_{n-1}\right\} .
$$

Thus, in order to prove $(\rightarrow)$, we just need to show

$$
\left\{t_{n-1}\right\} \rightarrow_{\hat{\mathcal{R}}}\left\{t_{n}\right\},
$$

whenever $t_{n-1} \rightarrow_{\mathcal{R}} t_{n}$. The computation step $t_{n-1} \rightarrow_{\mathcal{R}} t_{n}$ in the rewrite theory $\mathcal{R}$ can be expanded into the following rewrite sequence

$$
t_{n-1} \stackrel{r, \sigma, w}{\rightarrow} R, B \tilde{t}_{n-1} \rightarrow_{\Delta, B}^{*} \tilde{t}_{n-1} \downarrow_{\Delta, B}=t_{n}
$$

where $r=(\lambda \Rightarrow \rho$ if $C) \in R$. Here, we distinguish two cases according to the value of the position $w \in \mathcal{P} o s\left(t_{n-1}\right): w=\Lambda$ and $w \neq \Lambda$.

$(w=\Lambda)$ In this case, $t_{n-1}=_{B} \lambda \sigma$ and $\tilde{t}_{n-1}=_{B} \rho \sigma$ by the definition of $\rightarrow_{R, B}$. Furthermore, since $\mathcal{R}$ is topmost modulo $A x, \lambda \sigma$ and $\rho \sigma$ have sort Config. From these facts, it immediately follows that

$$
\left\{t_{n-1}\right\}={ }_{B}\{\lambda \sigma\} \stackrel{\hat{r}, \sigma_{\Lambda} \Lambda}{\rightarrow} \hat{R}, B_{B}\{\rho \sigma\}={ }_{B}\left\{\tilde{t}_{n-1}\right\} \rightarrow_{\Delta, B}^{*}\left\{\tilde{t}_{n-1}\right\} \downarrow_{\Delta, B}=\left\{t_{n}\right\}
$$

with $\hat{r}=\{\lambda\} \Rightarrow\{\rho\}$ if $C \in \hat{R}$. Hence, $\left\{t_{n-1}\right\} \rightarrow_{\hat{\mathcal{R}}}\left\{t_{n}\right\}$ when $w=\Lambda$.

$(w \neq \Lambda)$ Since $\mathcal{R}$ is topmost modulo $A x$ and $w \neq \Lambda$, there exist $u_{i} \in \mathcal{T}(\Sigma, \mathcal{V})_{\text {Config }}, i=1, \ldots, k$, with $k>1$ such that

$$
t_{n-1}=u_{1} \otimes \ldots \otimes u_{k}
$$

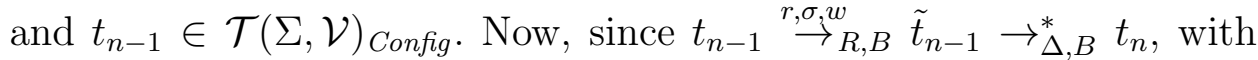
$A x=A C$ and $r=(\lambda \Rightarrow \rho$ if $C)$,

$$
\begin{aligned}
t_{n-1}=u_{1} \otimes \ldots \otimes u_{k} & ={ }_{A C} u_{\pi(1)} \otimes \ldots \otimes u_{\pi(m)} \otimes \lambda \sigma \\
\stackrel{r, \sigma, w}{\rightarrow} R, B & u_{\pi(1)} \otimes \ldots \otimes u_{\pi(m)} \otimes \rho \sigma \rightarrow_{\Delta, B}^{*} t_{n}
\end{aligned}
$$

where $1 \leq m \leq k-1$, and $\pi:\{1, \ldots, m\} \rightarrow\{1, \ldots, k\}$ is an injective function that selects a permutation of $m u_{i}$ 's within $u_{1} \otimes \ldots \otimes u_{k}$. Hence, we can build the following rewrite sequence

$$
\left\{t_{n-1}\right\}=\left\{u_{1} \otimes \ldots \otimes u_{k}\right\}={ }_{A C}\left\{u_{\pi(1)} \otimes \ldots \otimes u_{\pi(m)} \otimes \lambda \sigma\right\}
$$




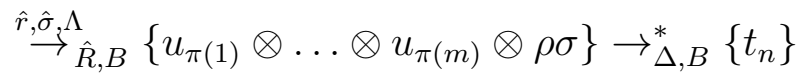

with $\hat{\sigma}=\sigma \cup\left\{X / u_{\pi(1)} \otimes \ldots \otimes u_{\pi(m)}\right\}$, and $\hat{r}=(\{X \otimes \lambda\} \Rightarrow\{X \otimes$ $\rho\}$ if $C) \in \hat{R}$. This proves that $\left\{t_{n-1}\right\} \rightarrow_{\hat{\mathcal{R}}}\left\{t_{n}\right\}$ also in the case when $w \neq \Lambda$.

Finally, by using the induction hypothesis A.2 and the rewrite step A.3, we easily derive the implication $(\rightarrow)$.

$(\leftarrow)$ Assume that $\left\{t_{i}\right\} \rightarrow_{\hat{\mathcal{R}}}^{*}\left\{t_{f}\right\}$, where $t_{i}$ and $t_{f}$ are arbitrary terms of sort Config. Then, $\left\{t_{i}\right\} \rightarrow_{\mathcal{R}}^{*}\left\{t_{f}\right\}$ is of the form

$$
\left\{t_{i}\right\}=\left\{t_{0}\right\} \rightarrow_{\hat{\mathcal{R}}} \ldots \rightarrow_{\hat{\mathcal{R}}}\left\{t_{n-1}\right\} \rightarrow_{\hat{\mathcal{R}}}\left\{t_{n}\right\}=\left\{t_{f}\right\}
$$

for some natural number $n \geq 0$. We proceed by induction on the length $n$ of the computation $\left\{t_{i}\right\} \rightarrow_{\hat{\mathcal{R}}}^{*}\left\{t_{f}\right\}$.

$n=0$. Immediate, since there are no rewrite steps.

$n>0$. This case is analogous to the proof of the inductive step of Case $(\rightarrow)$. By induction hypothesis, we have

$$
\left\{t_{i}\right\}=\left\{t_{0}\right\} \rightarrow_{\mathcal{R}}^{*}\left\{t_{n-1}\right\} \text { implies } t_{i}=t_{0} \rightarrow_{\mathcal{R}}^{*} t_{n-1} .
$$

Therefore, it suffices to show that $t_{n-1} \rightarrow_{\mathcal{R}}^{*} t_{n}$ and combine this result with the induction hypothesis to finally prove Case $(\leftarrow)$.

By hypothesis, $\left\{t_{n-1}\right\} \rightarrow_{\hat{\mathcal{R}}}\left\{t_{n}\right\}$, which can be expanded into the following rewrite sequence

$$
\left\{t_{n-1}\right\} \stackrel{\hat{r}, \hat{\sigma}_{\rightarrow} \Lambda}{\hat{R}, B}\left\{\tilde{t}_{n-1}\right\} \rightarrow_{\Delta, B}^{*}\left\{\tilde{t}_{n-1}\right\} \downarrow_{\Delta, B}=\left\{t_{n}\right\}
$$

where $\hat{r} \in \hat{R}$, and $\left\{t_{n-1}\right\},\left\{\tilde{t}_{n-1}\right\},\left\{t_{n}\right\} \in \mathcal{T}(\hat{\Sigma}, \mathcal{V})_{\text {State }}$. Observe that the first rewrite step of the rewrite sequence $(A .5)$ must occur at position $\Lambda$, since the rewrite theory $\hat{\mathcal{R}}$ is topmost.

Here, we distinguish two cases according to the form of the rewrite rule

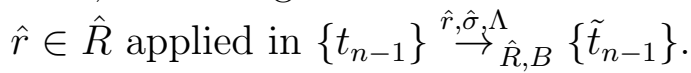

By Definition 6.4 $\hat{r}$ is either $\{\lambda\} \Rightarrow\{\rho\}$ if $C$ or $\{X \otimes \lambda\} \Rightarrow\{X \otimes \rho\}$ if $C$, as $A x=A C$ and $\left\{t_{n-1}\right\},\left\{\tilde{t}_{n-1}\right\} \in \mathcal{T}(\hat{\Sigma}, \mathcal{V})_{\text {State }}$. 
Case $(\hat{r}=(\{\lambda\} \Rightarrow\{\rho\}$ if $C))$. In this case, $\left\{t_{n-1}\right\} \stackrel{\hat{r}, \hat{\sigma}, \Lambda}{\rightarrow} \hat{R, B},\left\{\tilde{t}_{n-1}\right\}$ assumes the following form:

$$
\left\{t_{n-1}\right\}={ }_{A C}\{\lambda \sigma\} \stackrel{\hat{r}, \sigma, \Lambda}{\rightarrow}_{R, B}\{\rho \sigma\}={ }_{A C}\left\{\tilde{t}_{n-1}\right\} .
$$

Now, by Definition 6.4, $\lambda \sigma$ and $\rho \sigma$ are terms of sort Config; thus, we can also apply $r=(\lambda \Rightarrow \rho$ if $C) \in R$ to $\lambda \sigma$, thereby obtaining the following computation

$$
t_{n-1}={ }_{A C} \lambda \sigma \stackrel{r, \sigma, \Lambda}{\rightarrow}_{R, B} \rho \sigma \rightarrow_{\Delta, B}^{*}\left(\rho \sigma \downarrow_{\Delta, B}\right)=t_{n}
$$

which corresponds to $t_{n-1} \rightarrow_{\mathcal{R}} t_{n}$ when $\hat{r}=(\{\lambda\} \Rightarrow\{\rho\}$ if $C)$.

Case $(\hat{r}=(\{X \otimes \lambda\} \Rightarrow\{X \otimes \rho\}$ if $C))$. In this case, $\left\{t_{n-1}\right\} \stackrel{\hat{r}, \hat{\sigma}, \Lambda}{\rightarrow}_{R, B}$ $\left\{\tilde{t}_{n-1}\right\}$ must have the following form:

$$
\left\{t_{n-1}\right\}={ }_{A C}\{u \otimes \lambda \hat{\sigma}\} \stackrel{\hat{r}, \hat{\sigma}, \Lambda}{\rightarrow} \hat{R, B}\{u \otimes \rho \hat{\sigma}\}={ }_{A C}\left\{\tilde{t}_{n-1}\right\}
$$

where $u, \lambda \hat{\sigma}, \rho \hat{\sigma} \in \mathcal{T}(\Sigma, \mathcal{V})_{\text {Config }}$, and $\hat{\sigma}=\{X / c\} \cup \sigma$, for some substitution $\sigma$.

Now, by Definition 6.4, variable $X$ does not occur in either $\lambda$ or $\rho$; this implies that $\lambda \hat{\sigma}=\lambda \sigma$ and $\rho \hat{\sigma}=\rho \sigma$. Therefore, we can construct the following computation:

$$
t_{n-1}=A C u \otimes \lambda \hat{\sigma}=u \otimes \lambda \sigma \stackrel{r, \sigma, w}{\rightarrow}_{R, B} u \otimes \rho \sigma=u \otimes \rho \hat{\sigma}={ }_{A C} \tilde{t}_{n-1} \rightarrow_{\Delta, B}^{*} t_{n}
$$

where $r=(\lambda \Rightarrow \rho$ if $C) \in R$ and $w \in \mathcal{P}$ os $(u \otimes \lambda \sigma)$ is the position of the term $\lambda \sigma$ inside $u \otimes \lambda \sigma$. Hence, $t_{n-1} \rightarrow_{\mathcal{R}} t_{n}$ even in the case when $\hat{r}=(\{X \otimes \lambda\} \Rightarrow\{X \otimes \rho\}$ if $C)$.

Proof of Corollary 6.7 (sound topmost modulo $A x$ correction). Immediate by applying Theorem 5.6 to the topmost rewrite theory $\hat{\mathcal{R}}=(\hat{\Sigma}, \Delta \cup B, \hat{R})$.

\section{Proof of Corollary 6.17 (sound topmost $n$-extension correction).} The rewrite theory $\mathcal{R}_{n}=\left(\Sigma_{n}, \Delta \cup B, R_{n}\right)$ is topmost, so the result is immediate by Theorem 5.6 . 